\title{
Regards sur le
}

mouvement spirituel fondé par Sri Aurobindo et la Mère

Patrick Beldio

David Brême

Marie Horassius

Cahiers de

I'Institut Religioscope

Numéro 15

Septembre 2018

RELIGI@SCOPE
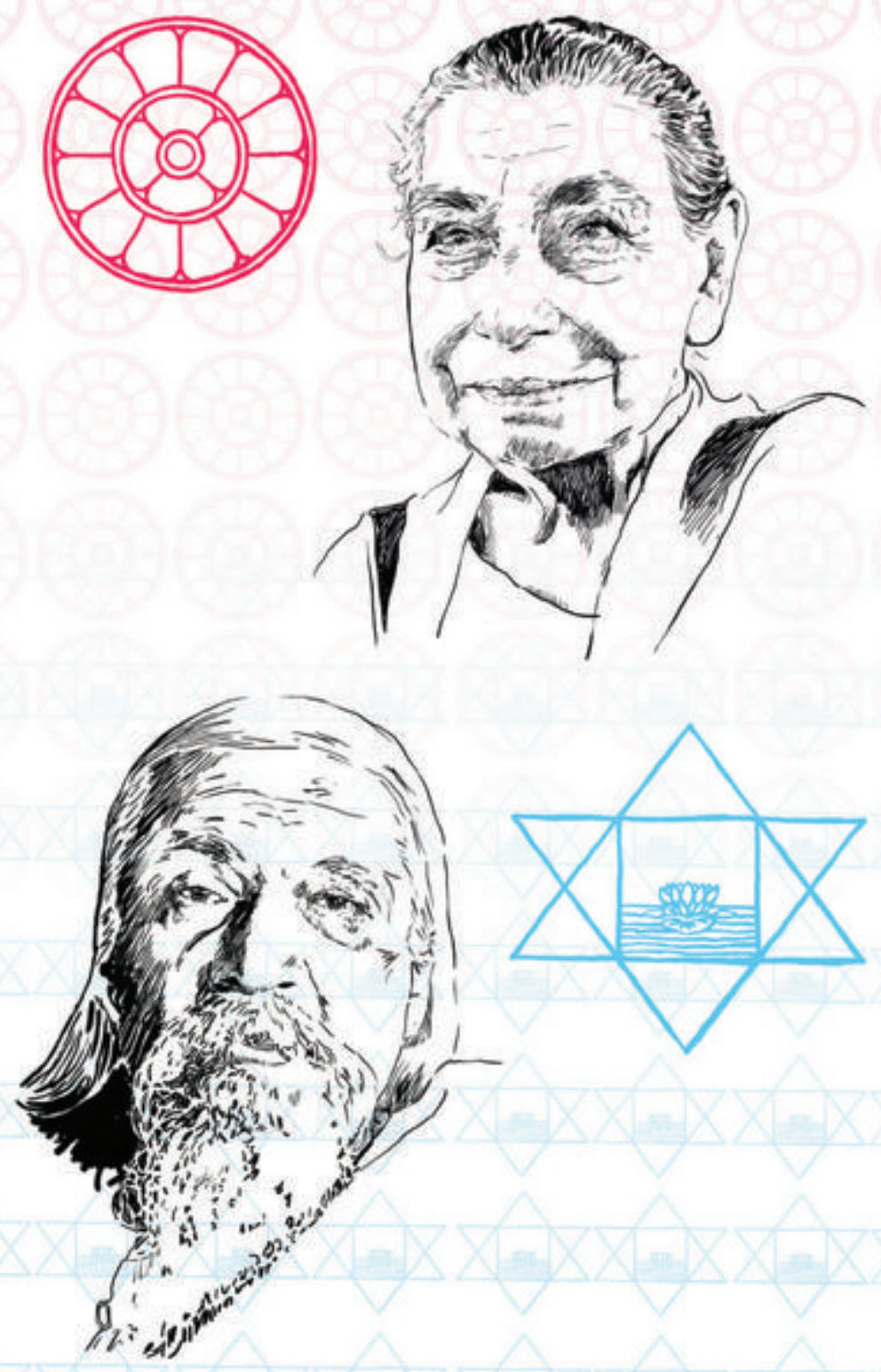
Les auteurs et leurs parcours académiques sont présentés à la fin du chapitre 1 .

L'illustration de couverture a été créée par Patrick Beldio ( 2018 Patrick Beldio).

Illustrations de Joan Brême de JOANOV', sœur de l'auteur et graphiste en France, qui accompagne au quotidien les structures à taille humaine pour toute leur communication imprimée comme digitale.

En savoir plus sur joanov. frl.

L'article de Patrick Beldio a été traduit de l'anglais au français par les soins de David Brême.

Ce cahier est également disponible en ligne au format PDF:

www.religioscope.org/cahiers/15.pdf

Ce cahier est distribué gratuitement et ne peut être vendu.

C 2018 Patrick Beldio - David Brême - Marie Horassius 


\section{Table des matières}

1. Présentation du mouvement spirituel Mère-Sri Aurobindo

David Brême

2. Contexte historique du mouvement spirituel Mère-Sri Aurobindo

David Brême

2.1. Biographie d'Aurobindo Ghose de 1872 à 1914

2.2. Biographie de Mirra Alfassa, dite la Mère (1878-1973), et de Sri Aurobindo de 1914 à 1950

3. Le yoga artistique de la Mère à l'Ashram de Sri Aurobindo

Patrick Beldio

3.1. L'Ashram de Sri Aurobindo à Pondichéry

3.2. Le yoga artistique de la Mère

3.2.1 La relation des disciples avec la « guru »

3.2.2 La peintre Huta, disciple de la Mère

3.2.3 Le « Supramental», le Nouveau Monde en couleurs vu par la Mère

4. Présentation du Centre Sri Aurobindo de Montréal

et de son hybridité culturelle indo-québécoise

David Brême

4.1. Introduction générale au Centre Sri Aurobindo de Montréal et à son contexte québécois

4.2. Le Centre Sri Aurobindo et sa construction communautaire québécoise (1965-1979) 
4.3. Institutionnalisation du Centre Sri Aurobindo et sa vocation (1979-2017)

5. Rituels et foi au cœur d'une utopie :

oscillations et négociations autour des pratiques croyantes à Auroville

Marie Horassius

5.1. Présentation d'Auroville et de son étude

5.1.1. Routinisation et charisme

5.1.2. L'indépendance d'Auroville contre la Sri Aurobindo Society

5.2. L'institutionnalisation "psychologique » du croire

5.3. Rituel collectif du Bonfire

5.4. Le Matrimandir

5.4.1. Matériellement

5.4.2. Les symboles 52

5.5. Les rituels individuels 54

5.5.1. Les signes d'appartenances 54

5.5.2. Les livres

5.5.3. Les photographies 54

5.5.4. Les prières et les citations 55

5.6. Conclusion 55

6. Références bibliographiques 
1 Présentation du mouvement spirituel

\section{Mère-Sri Aurobindo}

\section{David Brême}

Vient alors une période où l'abîme entre la convention et la vérité devient intolérable, où se lèvent des hommes doués de pouvoir intellectuel, les grands «avaleurs de formules" qui vigoureusement ou furieusement, ou avec la calme lumière de la raison, rejettent les symboles, les types et les conventions, renversent les murs de la prison et cherchent par la raison individuelle, par le sens moral ou les désirs du cœur, la Vérité que la société a perdue ou enterrée dans des sépulcres blanchis. Alors naît l'âge individualiste de la religion, de la pensée et de la société, l'âge du protestantisme est commencé, l'âge de la raison, l'âge de la révolte, du progrès et de la liberté. C'est encore une liberté extérieure et partielle; elle s'imagine encore, trompée par l'âge conventionnel qui l'a précédé, que la Vérité peut se trouver dans les formes extérieures, et elle rêve en vain d'une perfection déterminée par un mécanisme, mais c'est un passage nécessaire vers la période subjective de l'humanité où l'homme doit boucler le cercle et redécouvrir son moi profond en suivant une nouvelle ligne ascendante, ou une nouvelle révolution du cycle des civilisations.

Sri Aurobindo (1972a : 14-15)

\section{Introduction}

Définir un mouvement spirituel implique généralement d'en déterminer une période historique, des fondateurs, des valeurs, des croyances, des disciples, un développement, des structures matérielles et juridiques, en le réduisant à ce qui paraît stable et visible... Ces points de repère permettent de le situer dans un espace et un temps donné. Mais un mouvement ne s'y limite pas, particulièrement lorsqu'il est dit « spirituel », ce terme étant devenu un concept «valise » depuis la fin du XXe siècle.

Nous nous proposons d'étudier le mouvement spirituel fondé par Sri Aurobindo
(1872-1950) et la Mère (1878-1973) en présentant les dynamiques de trois de ses sites : l'Ashram de Sri Aurobindo à Pondichéry, Auroville au Tamil Nadu et le Centre Sri Aurobindo de Montréal. Nous qualifions ce mouvement de spirituel, car ce terme correspond à une catégorie interne propre aux discours de la Mère et de Sri Aurobindo. La Mère est le nom - à haute portée symbolique dans le contexte de l'Inde comme de l'hindouisme - donné par Sri Aurobindo à Mirra Alfassa, Française devenue sa partenaire mystique et la responsable de son ashram. 


\section{La Mère, une femme guru donnant des darshan}

Il n'est pas si fréquent qu'une femme occidentale ait été reconnue en tant qu'autorité spirituelle simultanément en Inde et en Occident. Certes, de façon plus contemporaine, des femmes guru indiennes ont acquis une notoriété en Occident. Mata Amritanandamayi, connue sous le nom d'Amma (née en 1953) ${ }^{1}$, est probablement la plus connue d'entre elles. Lors de rassemblements attirant des milliers de personnes en Europe, en Inde ou en Amérique, elle donne sa bénédiction sous la forme d'une accolade à chacune des personnes venant vers elle (Jean Huffer, 2010). D’autres femmes guru indiennes telles que Ma Anandamayi (1896-1982) donne leur bénédiction par le regard. Ce rituel de bénédiction se nomme darshan et consiste dans l'hindouisme en un échange visuel entre le dévot et la divinité, souvent figurée par une sculpture ou une icône. Depuis la fin du XIXe siècle ${ }^{2}$, il arrive que la divinité féminine soit une femme avatar s'adressant à tous ses dévots.

La Mère, alias Mirra Alfassa, a été une des rares femmes non indiennes à donner des darshan $^{3}$ en Inde, contribuant ainsi à ouvrir l'espace hybride d'une spiritualité indooccidentale en une sorte de symétrie aux guru venus en Occident. Par exemple, Mère Meera, Indienne vivant actuellement en Allemagne, reconnaît avoir été inspirée par la Mère dans son propre parcours de femme guru (Adilakshmi, 1987 : 9-15). La Mère elle-même a formé avec Sri Aurobindo une relation spirituelle mystique assez inédite, tous deux ayant été mus par un puissant désir d'émanciper l'humanité de ses souffrances par l'amour du divin 4 . Sri Aurobindo a probablement été inspiré dans le choix de nommer Mirra Alfassa " la Mère » par le couple mythique du yogi Ramakrishna (1836-1886) et de son épouse Sarada Devi (1853-1920)5 , une des premières femmes à avoir tenu publiquement ${ }^{6}$ un darshan en Inde ${ }^{7}$ sans distinction de genre, de classe ou de caste. Toutefois, le couple de Ramakrishna et Sarada Devi, réputé chaste, a été religieusement institué, tandis qu'aucune institution maritale n'a relié Sri Aurobindo et la Mère. Par ailleurs, il a été difficile pour Sarada Devi comme pour Ma Anandamayi (Aymard, 2009) - un peu plus jeune que la Mère - de manifester leur autorité spirituelle en Inde, au

${ }^{1}$ Amma.org, consulté le 19 décembre 2017.

2 On peut considérer que Sarada Devi (1853-1920), épouse de Ramakrishna, est le prototype d'une femme avatar incarnant la Déesse.

3 Pour aller plus loin au sujet du darshan, lire Eck, 1998.

${ }^{4}$ Lire le poème épique Savitri de Sri Aurobindo pour avoir une idée de ce que revêt comme sens cet amour pour Sri Aurobindo.

5 Sarada Devi a été rituellement instituée représentante de «la Mère divine » en 1893 par son époux, également prêtre-brahmane du temple de Kālī de Dakineshwar, au nord de Kolkotta, autrefois Calcutta.

${ }^{6}$ Lynn Teskey Denton (2004 : 147) cite par exemple au Kerala les cas de Chanikrottu Amma au XIVe siècle et de Kurur Amma au XVIe siècle (Amma voulant dire "mère »), mais celles-ci semblent avoir été relativement peu reconnues publiquement au cours de l'histoire, jusqu'à leur émergence au XIXe siècle.

7 Il faut dire que le caractère unifié de l'Inde sur les plans politique, administratif et pratique - notamment par un exceptionnel réseau de chemin de fer sur le plan pratique - est relativement récent, eu égard aux centaines de royaumes qui ont précédé le Raj britannique. 
sein d'une tradition hindoue où la transmission s'effectue principalement de guru à disciple masculin. Ainsi, Vivekananda (1863-1902) prit le relais de son guru Ramakrishna, mais mit quelque temps (Goulet, 2010) à intégrer Sarada Devi dans la Mission Ramakrishna après le décès de son guru tandis que ce dernier l'avait reconnue comme son égale.
Dès lors, le fait que la Mère, Française d'origine ${ }^{8}$, ait été établie comme responsable d'un ashram dès 1926 est exceptionnel, dans la mesure où les figures d'autorités religieuses hindoues ou non hindoues sont institutionnellement représentées en Inde par des hommes, même si, dans certains textes ${ }^{9}$ hindous, les dieux sont soumis à la Déesse.

\section{Le yoga de Sri Aurobindo et de la Mère dans le contexte hindou}

L'hindouisme désigne étymologiquement les religions pratiquées à l'est du fleuve Indus. Historiquement, cette dénomination est étroitement reliée à une définition coloniale englobante créée par l'empire turco-mongol au Moyen-Âge, puis par l'Empire britannique. Les religions du quasi-continent indien ont été et sont très variées : elles ne correspondent pas à l'idée commune occidentale de la religion en tant que monothéisme référant à des textes révélés et des autorités centralisées ${ }^{10}$. La structure sociofamiliale hindoue est hiérarchisée en termes de castes ou varṇavyavasthā (brähmaṇa, la caste la plus haute des religieux ou varna des officiants ; kșatriya, la caste de la gouvernance ou varṇa des guerriers ; vaiśya la caste ou varṇa des commerçants, des employés, de la classe laborieuse et enfin, au bas de l'échelle sociale, les śüdra appartenant au varṇa des serviteurs) et de corps de métiers transgénérationnels ou jātī. Ces liens socioreligieux sont bien plus prépondérants que des dénominations de divinités spécifiques pour rendre compte de l'hindouisme (Dumont, 1971), lequel peut changer radicalement d'une province à l'autre. Par ailleurs, le temple s'insère dans la dynamique cultuelle de cette organisation socioreligieuse : ce dernier peut être présent en tant qu'édifice local, en tant qu'autel à la maison ou comme lieu de pèlerinage.
Quatre buts de l'existence (purușārtha) sont généralement considérés dans l'hindouisme : le désir (kāma), la prospérité matérielle (artha), le devoir (dharma) et la libération (moks $a)$. Les deux premières phases, nécessaires à la perpétuation de la société sont assumées par le chef de famille (grhastha) pour l'aspect artha. Néanmoins, le désir des plaisirs et la volonté de prospérité reconduisent les souffrances (saṃsāra), même si elles peuvent être temporisées en effectuant et en assumant son devoir social (dharma). Pour échapper au cycle de la souffrance, des pratiques yogiques conduisent à la mokșa (la libération), le véritable but de l'hindouisme brahmanique, existant au moins depuis le Ve siècle avant Jésus-Christ. Pour atteindre ce but, une classe hors-caste de la société hindoue, soit les yogis, les sādhu, etc., s'engage dans une vie ascétique, en renonçant aux normes de la famille hindoue et de la vie socioprofessionnelle qui incombent par devoir à chaque jātí (corps de métier). Traditionnellement, les renonçants ne le deviennent qu'une fois accompli leur devoir familial et social. Toutefois, dans les faits, ce renoncement peut avoir lieu à tout âge. Ce sont ces ascètes marginaux - mais ayant le rôle fondamental de manifester l'idéal religieux de

8 Dans la tradition hindoue, ceux qui viennent du dehors de l'Inde étaient considérés encore récemment comme impurs.

9 Devī-Māhātmya, Devī Bhāgavata Purāna, etc.

10 Même si les protestantismes et l'islam varient déjà sur ce dernier point. 
toute la société - qui ont souvent fait la fascination de l'Inde auprès d'Occidentaux.

Ces communautés ascétiques hindoues, parfois organisées en ashram autour d'un guru un sādhu ${ }^{11}$ devenu "professeur» - suivent généralement des règles définies structurant leur transmission, dont celle de la désignation du successeur de la lignée ascétique par le fondateur. Tel a été le fonctionnement de la transmission religieuse formant des traditions dans nombre de lignées shivaïtes, vishnouistes, bouddhistes tibétaines, etc..

En préconisant une pratique assidue du yoga, la chasteté (brahmacharya) et une hygiène de vie spécifique à ses disciples, en se référant aux rishi de l'époque védique - sorte de " clairvoyants » ayant transmis les hymnes du Veda - le mouvement spirituel de la Mère et de Sri Aurobindo s'inscrit aussi de facto dans ce vaste courant ascétique qu'a produit l'hindouisme, mais, à sa différence, ne propose ni de lignée à perpétuer ni de fonder un culte qui serait un "aurobindoïsme ». Il y a là un point de rupture fondamental avec les traditions ascétiques de l'Inde. Même si Sri Aurobindo a reçu un enseignement du yogi Baskar Lélé un an avant d'être emprisonné par les Anglais et a désigné la Mère en tant que guide de ses disciples, il se considérait lui-même comme un avatar dont il était la facette masculine agissant en coopération avec la Mère, sa facette féminine. Selon Véronique Altglas (2005), dans le cas du nouvel hindouisme du Siddha yoga récemment implanté en Occident, sa légitimité relève davantage du choix et de l'engouement des disciples que de son institution par une hiérarchie séculaire. Analogiquement, on pourrait dire que la légitimation du Mouvement spirituel Mère-Sri Aurobindo procède davantage du charisme de ses fondateurs, de la nouveauté de leur vision et de la réception de leurs disciples que d'une lignée définie. Par exemple, la finalité de ce mouvement spirituel est la divinisation de

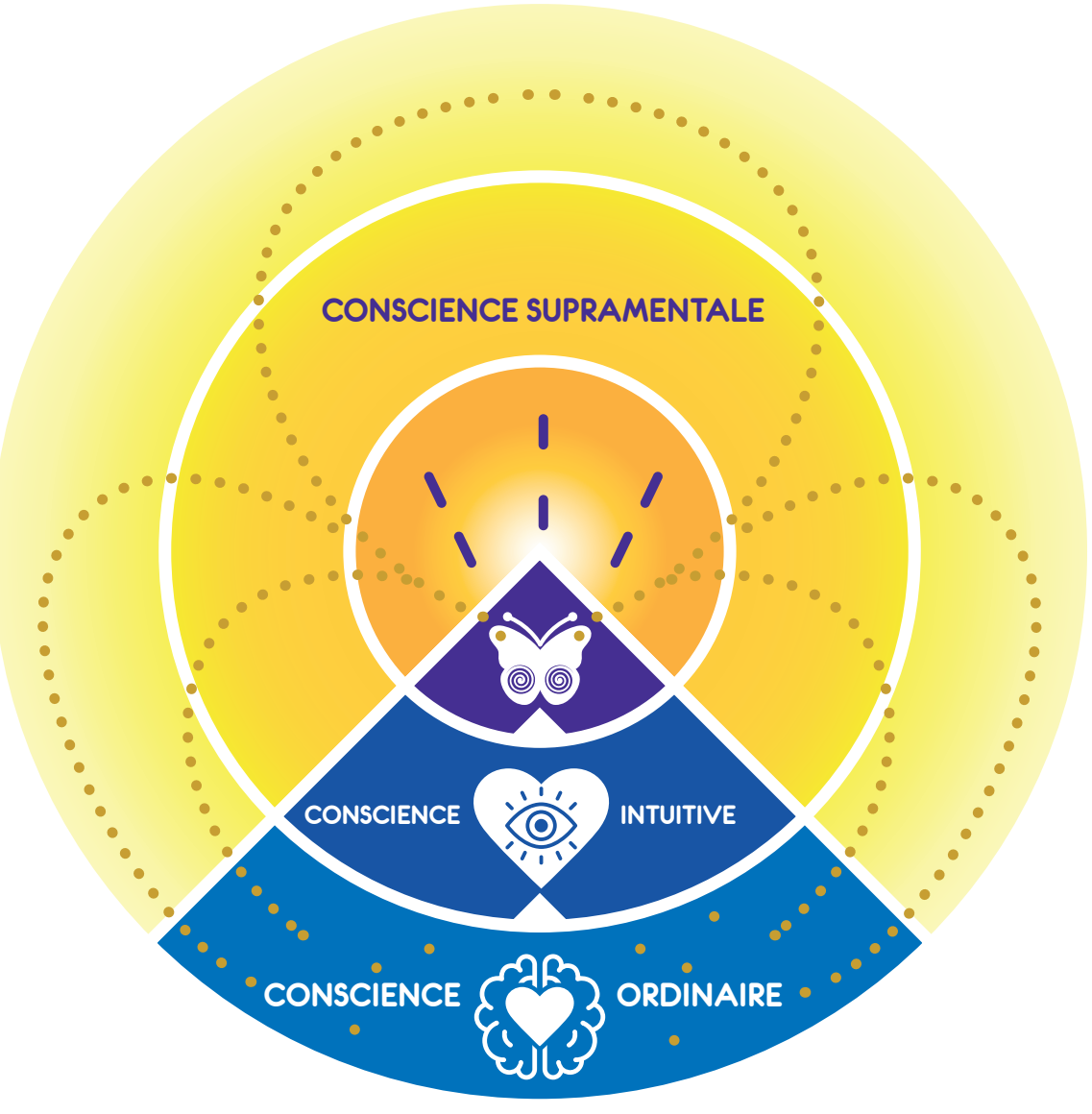

${ }^{11}$ Le guru transmet, enseigne et est sédentaire, tandis que le sādhu et le sannyāsin sont itinérants. 
la matière par la pratique d'un yoga intégral (Pūrṇa-yoga). Sri Aurobindo déclare commencer son odyssée de divinisation de la matière à partir du stade de la libération (mokșa), but ultime de l'hindouisme. Il en rend ainsi compte:

« Par comparaison avec les anciens yogas, celui-ci est nouveau :

Parce qu'il ne vise pas à nous faire sortir du monde et de la vie pour nous conduire au ciel ou au nirvâna, mais qu'il se propose une transformation de la vie et de l'existence, non pas incidemment ou à titre subordonné, mais comme but central et distinct. [...]

Parce que le but recherché n'est pas l'obtention individuelle de la réalisation divine pour le profit de l'individu, mais quelque chose à gagner pour la conscience terrestre ici-bas [...]. Ce qu'il y a à gagner aussi, c'est l'obtention d'un Pouvoir de conscience (le Supramental) qui n'est pas encore organisé ni directement actif dans la nature terrestre ou même dans la vie spirituelle, mais qui reste à organiser et à rendre directement actif. » (1976: 69-70)

Le premier point mentionné par Sri Aurobindo de la recherche d'une conscience nouvelle - ou Supramental - pour qu'elle se manifeste ici-bas pourrait être schématisé ainsi qu'on le voit sur l'illustration de la page précédente ${ }^{12}$.

Le changement de paradigme opéré par Sri Aurobindo et la Mère par rapport à un hindouisme défini comme une quête de la libération du cycle des existences - supposant en corollaire la croyance en la réincarnation - est qu'il s'agit d'amener cet état de paix et de béatitude yogique sur la terre, jusqu'au cœur de la conscience mentale, psychique, vitale et physique de l'être humain et de la matière en général. Cela a été symbolisé par la présence de points lumineux dans le monde ordinaire de la conscience, soit le triangle dans le schéma cidessus. Sri Aurobindo a établi la pensée et la métaphysique de ce nouveau paradigme en réinterprétant les Veda, les Upanishad, la Bhagavad-Gìtā et en formulant sa propre pensée, accessible par ses livres La vie divine, La synthèse des yogas, La manifestation $d u$ supramental sur la terre, le livret La Mère et le plus long poème en langue anglaise : Savitri.

La Mère, cherchant à accomplir dans la matière l'idéal formulé par Sri Aurobindo, narre quant à elle dans L'Agenda de Mère la pratique de son "yoga des cellules ", consistant à révéler l'aspect divin de la cellule par une attention donnée constamment à la force-conscience du supramental «descendant» dans son corps. Cette aventure intérieure microcosmique a été racontée de façon vivante dans le flux du quotidien par son disciple Satprem.

La transmission spécifique du mouvement spirituel de la Mère et de Sri Aurobindo est représentée ici avec une croissance de l'importance du charisme requis, étant donné
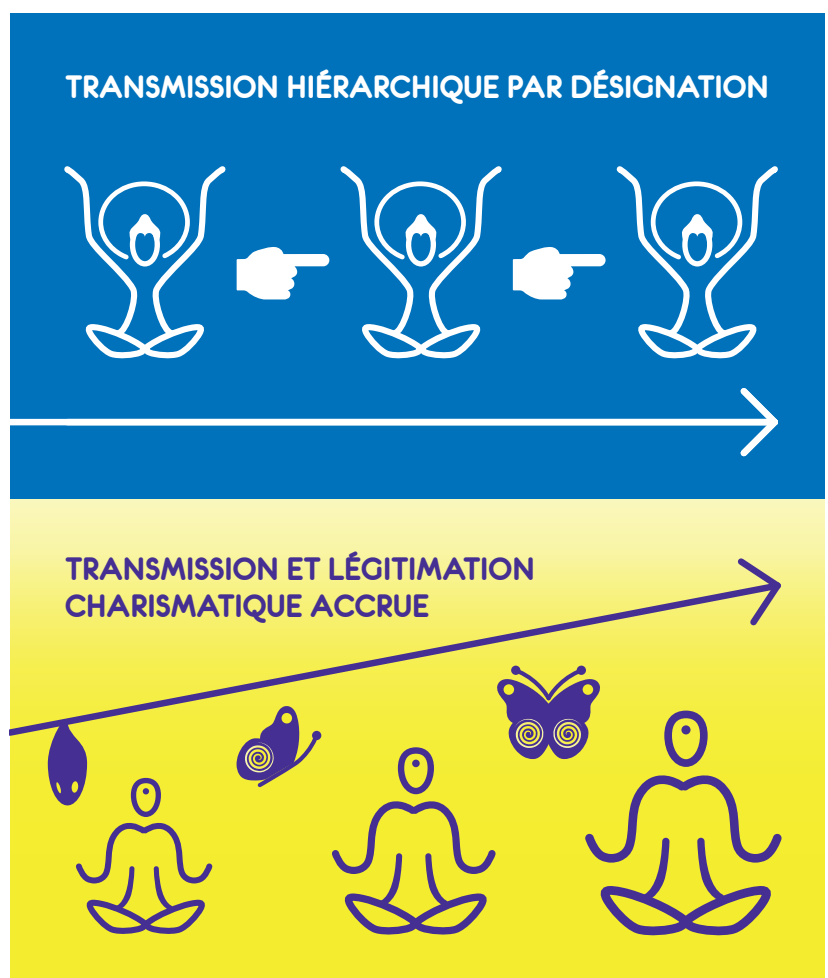

12 Les icônes utilisées ont été créées avec une finalité didactique visuelle et sont sans lien direct avec les symboles officiels du Mouvement spirituel Mère-Sri Aurobindo. 
que Sri Aurobindo ne fut pas désigné par Ramakrishna, Vivekananda ou Baskar Lélé pour leur succéder et du fait de l'absence de désignation de successeurs par la Mère. Ultimement, ce mouvement spirituel a pour légitimation - ou faillite de sa légitimation - la capacité de ses membres à incarner les qualités divines décrites par la Mère et Sri Aurobindo et à accomplir l'odyssée de La manifestation du Supramental sur la terre (Sri Aurobindo, 1974).

Ce passage d'une hiérarchie traditionnelle à une hiérarchie se construisant dans la valorisation d'un futur harmonieux, présente certaines tensions dans les différents sites de ce mouvement. En effet, ce changement d'horizon suppose de pouvoir en partie rejeter et transformer des structures passées, ce que chaque communauté (l'Ashram de Sri Aurobindo à Pondichéry, Auroville et le Centre Sri Aurobindo de Montréal) articule selon son alchimie propre. De plus, ce mouvement a aussi été rendu possible par son inscription initiale à Pondichéry et son entrelacs culturel francoindien. Les identités interculturelles de Pondichéry, de la Mère et de Sri Aurobindo ont favorisé la globalisation de ce mouvement spirituel de différentes manières au fil du temps.

\section{Bref historique du mouvement spirituel Mère Sri Aurobindo}

Une chronologie d'ensemble met en parallèle l'histoire du mouvement à l'intérieur du cercle et les événements majeurs historiques du monde à l'extérieur du cercle.

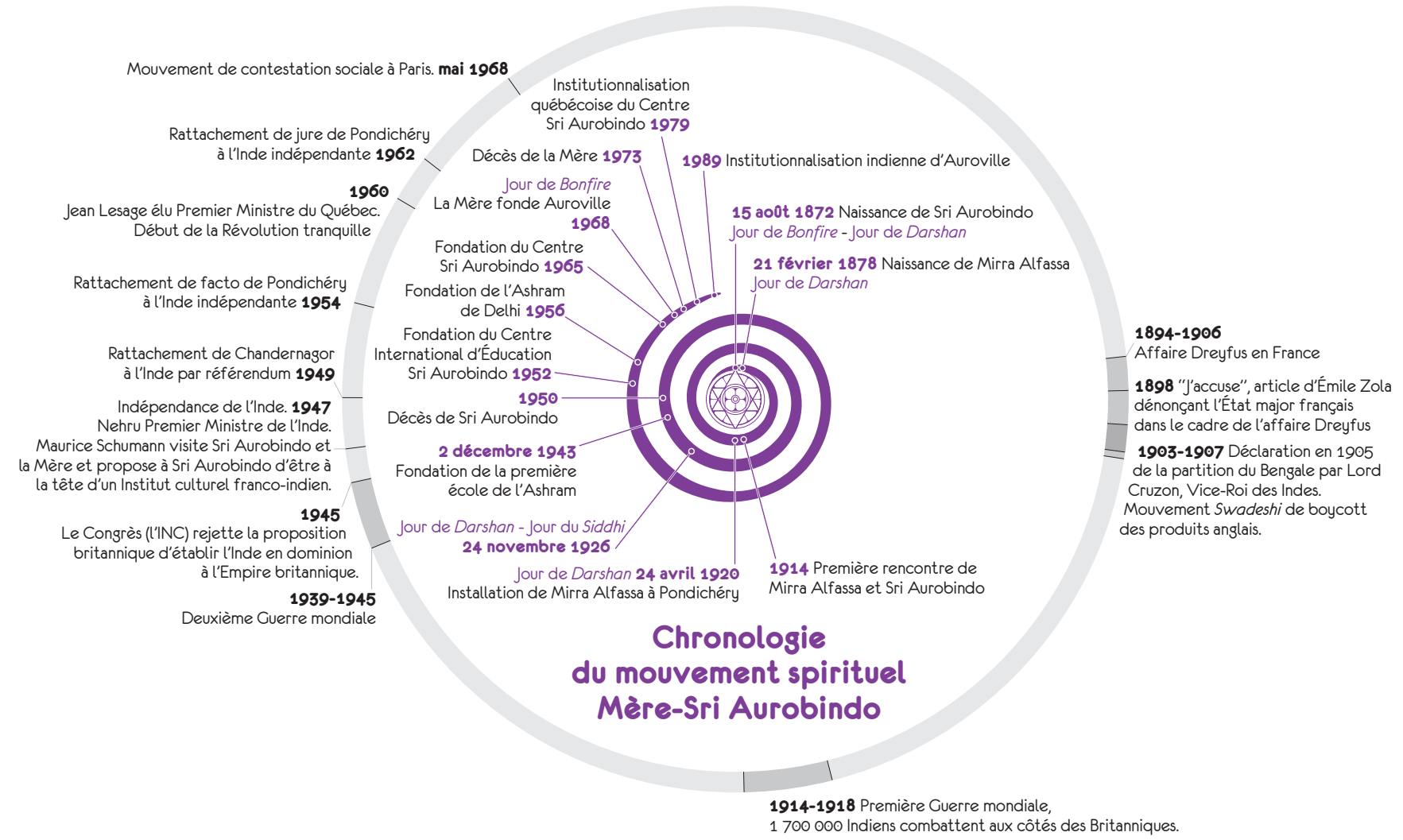


Larrivée de Sri Aurobindo en 1910 à Pondichéry avec quelques-uns de ses compatriotes bengalis ayant lutté pour l'indépendance de l'Inde ancra dans une forme d'exil ce mouvement spirituel, qui devint manifeste en 1914 lorsque Mirra Alfassa rencontra Sri Aurobindo. Ils publièrent alors avec Paul Richard ${ }^{13}$ la revue Arya, dont la portée interculturelle était exprimée par le bilinguisme anglo-français des parutions. Les premières œuvres majeures de Sri Aurobindo furent publiées dans cette revue, notamment $L a$ Synthèse des Yogas (1914/1970) et Le Secret du Veda (1914/1989). À cette occasion, Ackroyd Aravinda Ghose prit officiellement le nom de Sri Aurobindo (Van Vrekhem, 2007 : 157).

Un deuxième coup d'envoi du Mouvement spirituel Mère-Sri Aurobindo fut l'installation définitive de Mirra Alfassa à Pondichéry en 1920.

Il s'ensuivit en 1926 la fondation officielle de l'ashram Sri Aurobindo à Pondichéry. Cette date correspond à la désignation de Mirra Alfassa par Sri Aurobindo en tant que la Mère suite au jour du Siddhi14- commémoré annuellement par un Darshan - et en tant que guru de ses disciples. Cet ashram était initialement prévu pour des disciples ayant renoncé au monde, des hommes dits ashramites, consacrés exclusivement à la réalisation du yoga et des desseins de Sri Aurobindo et de la Mère. La Mère dit en 1971 de l'Ashram qu'il « est la conscience centrale» (1991: 77) de ce Mouvement spirituel. Comme dans la plupart des ashrams en Inde, les aspirants devaient être célibataires ou ne pas avoir de charge familiale, ne pas se marier, porter un vêtement traditionnel blanc et il leur était en général interdit d'entrer en relation avec le monde extérieur à l'ashram. Ces conditions sociales et statutaires évoluèrent toutefois du fait de la migration de familles bengalies pendant la Seconde Guerre mondiale. Un article de Patrick Beldio en rend plus précisément compte en mettant en exergue la guidance spirituelle artistique picturale de la Mère envers une de ses disciples.

La période succédant au décès de Sri Aurobindo en 1950 fut à la fois difficile et féconde. Un des défis de cette époque tenait au contexte spécifique du territoire de Pondichéry, rattaché tardivement à l'Inde indépendante, de facto en $1954^{15}$. Du point de vue de certains disciples tels que Dilip Kumar Roy, la Mère n'avait pas la légitimité de diriger l'Ashram sans Sri Aurobindo, ce qu'il rapporta au Premier ministre Nehru en 1951. Son rapport n'était probablement pas étranger - sur le plan politique - à la revendication d'une Inde indienne et au rattachement de Pondichéry à celle-ci. Toutefois, Nehru avait la volonté pacifique, mais déterminée d'établir dès 1947 Pondichéry en « fenêtre par où la France et l'Inde pourraient communiquer »(Weber, 1996)16. Ainsi, deux visites de Nehru à l'Ashram de Sri Aurobindo à Pondichéry en 1955 et 1963 scellèrent à la fois le rattachement de Pondichéry à l'Inde et la pleine légitimité du leadership de la Mère sur cet ashram (Satprem, 1979 : 180).

\footnotetext{
13 Paul Richard, alors marié à Mirra Alfassa, en eut la responsabilité légale jusqu’en février 1915, date de son départ pour la France et à partir de laquelle il remit cette responsabilité à Sri Aurobindo.
}

14 «Le jour du "Siddhi”, la Mère aurait demandé à Krishna de s'incarner en Sri Aurobindo et aurait relié chacun des disciples de Sri Aurobindo présents à une divinité spécifique relevant d'une forme de conscience nommée "Overmind". Sri Aurobindo aurait ensuite demandé à la Mère de renoncer à manifester ces formes de "l'Overmind", afin d'aspirer à manifester une conscience divine le transcendant. » (Brême, 2018 : 70)

15 Les comptoirs et établissements coloniaux français de l'Inde ont été rattachés de jure par le Parlement français et le Général de Gaulle en 1962, sauf Chandernagor qui s'est rattaché à l'Inde indépendante par référendum dès 1949.

16 Nehru écrivit par exemple le 1er novembre 1954 dans La Gazette de l'État de Pondichéry, Karikal, Mahé et Yanaon : « Je suis heureux de savoir que Pondichéry continuera à rester un centre de culture et de langue française, un lien culturel qui unira la République française et la République indienne ». 
Entre-temps, l'Ashram de Sri Aurobindo à New Delhi fut fondé en 1956 en tant que branche officielle de l'Ashram de Sri Aurobindo à Pondichéry, ce qui était un indicateur des échanges accrus entre ce mouvement spirituel et la capitale de l'Inde. Le 29 février 1956 représente aussi une date mythique importante pour ce mouvement spirituel, car elle correspondrait, selon la Mère, à la première « manifestation du Supramental sur la terre [qui ne serait] plus seulement une promesse, mais un fait vivant, une réalité » (Satprem, 1978 : 76).

En 1965, une autre expansion du Mouvement fut la création du Centre Sri Aurobindo à Montréal à l'initiative de la Québécoise Madeleine Gosselin, avec la bénédiction de la Mère. Madeleine Gosselin souhaitait faire du Centre Sri Aurobindo de Montréal une branche de l'Ashram de Pondichéry, en respectant sa charte, au même titre que celui de Delhi. Toutefois, son développement fut en grande partie autonome $\mathrm{du}$ fait de l'éloignement géographique et des différences de mentalité. Un article en rend compte dans le présent cahier. D’autres centres furent fondés à travers le monde, au Kenya, à Singapour et aux États-Unis, notamment en Californie $^{17}$ et à New York ${ }^{18}$.

À cette époque, la Mère eut plus particulièrement à cœur de réaliser Auroville, fondée par elle en 1968 et située à dix kilomètres au nord de Pondichéry. La Mère disait d'Auroville :

«Enfin un endroit où l'on pourra ne penser qu'à l'avenir.

Enfin un endroit où l'on pourra ne penser qu'à progresser et à se surmonter soimême.

Enfin un endroit où l'on pourra vivre en paix, sans conflits et sans rivalités des nations, de religions et d'ambitions.
Enfin un endroit où rien n'aura le droit de s’imposer comme vérité exclusive.

La terre a besoin d'un endroit où les hommes puissent vivre à l'abri de toutes les rivalités nationales, de toutes les conventions sociales, de toutes les moralités contradictoires et de toutes les religions antagonistes; un endroit où, libérés de tous ces esclavages du passé, les êtres humains pourront se consacrer totalement à la découverte et à la mise en pratique de la Conscience Divine qui veut se manifester. Auroville veut être cet endroit et s'offre à tous ceux qui aspirent à vivre la vérité de demain. " (La Mère, $1991: 1)$

La réalité actuelle d'Auroville sera présentée par l'article de Marie Horassius. À titre contextuel, il faut mettre en relief que les années soixante furent marquées par l'intérêt que suscitait l'imaginaire de l'Inde auprès de la jeunesse européenne et des courants beatnik et hippie en Amérique du Nord... et soixantehuitard dans le cas de la France. Cette ouverture des baby-boomers à l'imaginaire de l'Inde avait déjà avait été précédée par l'ouverture orientaliste savante ou romantique francoallemande du XIXe siècle (Hulin, 1996), mais elle coïncidait pendant les sixties et les seventies avec un rejet des structures traditionnelles socioreligieuses occidentales. Cette jeunesse idéalisait aussi à sa manière un Orient en grande partie réinventé (Saïd, 2005). Ainsi, les sites du Centre Sri Aurobindo à Montréal et d'Auroville, créés principalement par des Occidentaux après 1960, ont nécessairement formé une construction culturelle hybride entre la culture occidentale de cette génération et la culture indienne de cette époque en plein renouveau depuis son indépendance. Le site de l'Ashram de Sri Aurobindo à Pondichéry a hérité quant à lui d'une structure plus hiérarchisée mise en place

17 https://sriaurobindocenterla.wordpress.com/center/; http://sasp.collaboration.org/

$18 \mathrm{http}: / /$ matagiri.org 


\section{Relations entre les différents sites étudiés et autres institutions}

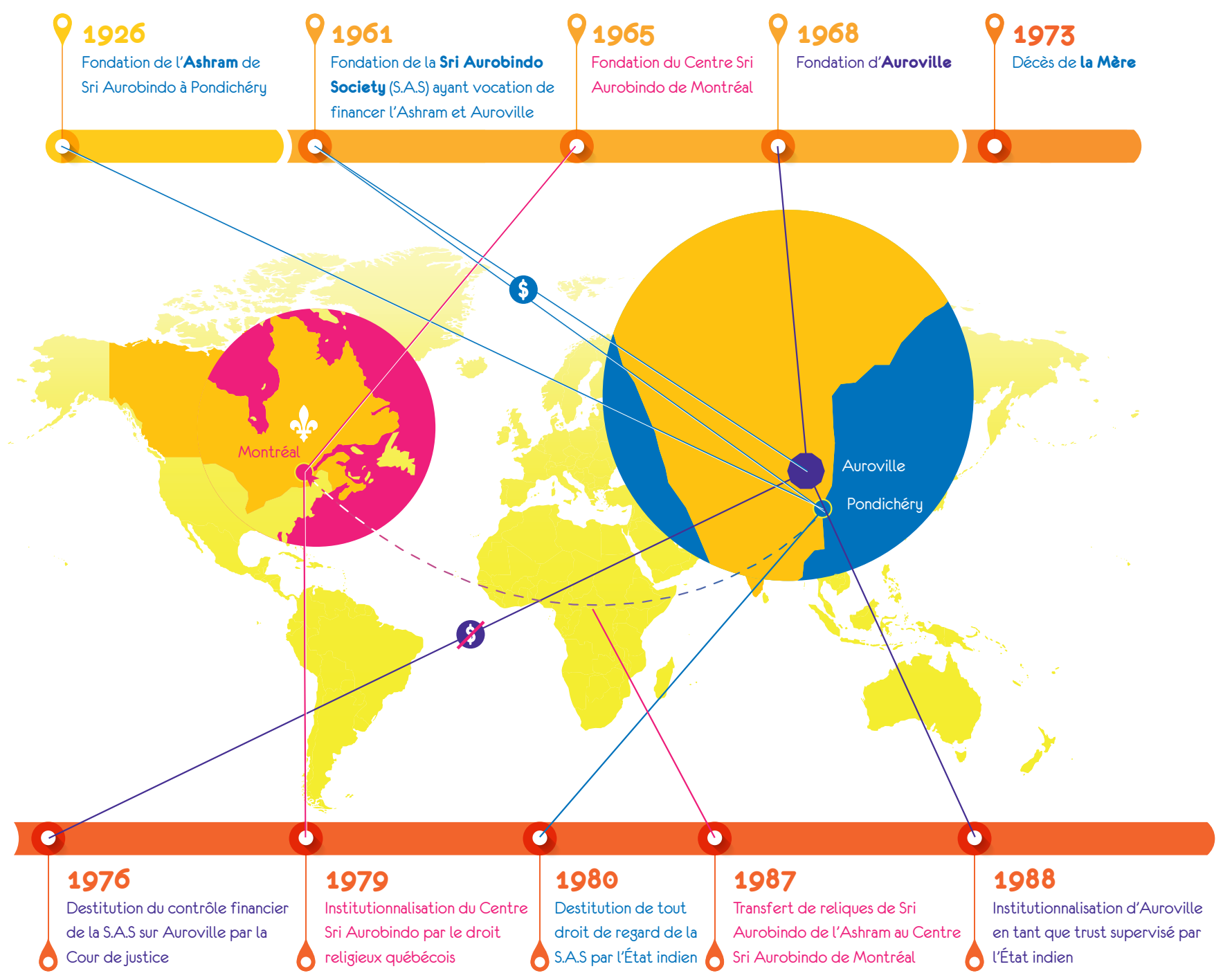

par la Mère, mais novatrice comme le montre Patrick Beldio dans son article. Les relations entre ces sites n'ont pas toujours été aisées du fait de ces décalages socioculturels, d'autant plus que la Sri Aurobindo Society, organisme fondé en 1962 par la Mère pour financer Auroville et l'Ashram de Sri Aurobindo à Pondichéry, avait un président qui, au décès de la Mère, souhaitait prendre le contrôle de ce qu'elle avait fondé. Des interventions successives des partisans du projet international d'Auroville puis de l'État indien, destituèrent cet organisme de ses prétentions. Le schéma historique ci-dessus rend compte d'une partie de la complexité relationnelle existante entre ces différents sites et institutions.
La contribution de ce cahier sur le Mouvement spirituel Mère-Sri Aurobindo consiste à proposer un état des lieux à travers des études portant sur les trois sites déjà mentionnés. Mais ce mouvement spirituel ne se limite pas à ces trois sites. Les nombreuses publications papier et en ligne de l'Ashram de Sri Aurobindo, des Éditions Albin Michel et des Éditions Robert Laffont ont notamment permis une vaste diffusion de la pensée de Sri Aurobindo, dont la réception varie d'un lecteur à l'autre. L'intérêt d'une étude se focalisant sur des sites explicitement consacrés à la spiritualité de la Mère et de Sri Aurobindo réside dans le fait que de nombreuses personnes y ont engagé leur vie et qu'ils sont plus descriptibles. 


\section{Les auteurs}

Trois spécialistes de ce mouvement ayant récemment présenté leurs thèses à ce sujet ou étant en train de l'achever s'accordent pour reconnaître la légitimité charismatique des fondateurs Sri Aurobindo et la Mère, en empruntant ce concept au sociologue Max Weber. Chacun a toutefois développé sa perspective spécifique.

- Patrick Beldio, professeur d'art et de sculpture à Washington ayant terminé son doctorat en 2016 sur L'Art et la beauté, l'opposition et la croissance dans l'Ashram de Sri Aurobindo, a effectué un terrain de recherche à l'Ashram de Sri Aurobindo à Pondichéry en 2012-2013. Sa perspective se fonde sur ce que David Morgan formule dans le champ de l'art visuel comme un " sacred gaze », un regard sacré. En effet, au milieu de ce qu'on appelle un tournant matériel (Pattinson, 2007) dans l'étude de la religion, il y a un mouvement de déconstruction de l'analyse dominante centrée sur le texte et donc fondée sur l'herméneutique et la sémantique ${ }^{19}$. Un objectif primordial de ces chercheurs est de construire une approche matérielle de l'étude de la religion, qui ne remplacerait pas les textes, mais augmenterait et transformerait l'enquête de manière à rendre compte de la présence et de l'utilisation des objets physiques par les corps humains, les valeurs, les idées et les croyances «immatérielles " d'une communauté donnée. Ils cherchent également à comprendre la nature de la vision, culturellement imbriquée dans l'utilisation des objets de culture visuelle et d'art, c'est-à-dire comment les êtres humains expérimentent leur sens visuel et comment cela influence la formation de l'identité dans des contextes culturels spécifiques. Cette méthodologie se fonde sur cette question : quel pouvoir les images ont-elles «pour effrayer, séduire, tromper, influencer et inspirer?» (Morgan, 2005 : 259). En d'autres termes, cette approche cherche à découvrir ce que les images font aux personnes: comment chacun les crée et les utilise pour mettre cette identité en action. Dans cet article, Patrick Beldio s'intéresse à la culture visuelle de la religion et au genre de vision qui se produit dans le contexte de la pratique et de l'expérience religieuse, ce qu'on a appelé la «piété visuelle» (Morgan, 1998) : comment la production et l'utilisation d'images aident-elles les communautés à créer et à maintenir une vision du monde partagée qu'elles considèrent comme sacrée, une identité sacrée qu'elles veulent protéger et / ou diffuser? Quels usages rituels des images emploient-elles pour atteindre cette identité ? Patrick Beldio en rendra compte à travers « le yoga artistique de la Mère à l'Ashram de Sri Aurobindo ", principalement en relation à sa disciple Huta. Son idée d'un yoga artistique de la Mère s'appuie sur des analyses antécédentes (Apostolos-Cappadona, 1980 ; Cenkner, 1984) de la relation entre l'art et la spiritualité dans la pensée de Sri Aurobindo.

- Marie Horassius, doctorante à l'EHESS, a effectué plusieurs terrains de recherche à Auroville depuis 2012, communauté d'environ 2700 personnes en 2017. À la lumière des données de son propre terrain, son approche se situe dans une proposition de réexaminer l'analyse wébérienne du sociologue Stuart Leard (1993) sur l'orientation mystique intramondaine des Auroviliens pensée comme

19 C'est ce que Birgit Meyer (2012 : 35) appelle «l'approche mentaliste enchâssée dans les généalogies dominantes » de l'érudition. 
forme de résistance à la routinisation ou quotidianisation. Ces derniers termes traduisent un concept de Max Weber désignant une phase, où suite au décès du fondateur charismatique d'un mouvement, celui-ci se "normalise", se donne des règles et s'institutionnalise. Marie Horassius nuance dans son article intitulé « Rituels et foi au cœur d'une utopie : oscillations et négociations autour des pratiques croyantes à Auroville " cette analyse critique de Leard pour souligner une institutionnalisation "psychologique » du croire à Auroville, malgré l'absence de religion présente ou de déclaration de son absence. Pour ce faire, elle s'appuie sur différents travaux produits sur Auroville (Leard, Minor, Pillai, Jouhki, Meier) et sur des auteurs en sciences des religions tels que Mircea Eliade et Albert Piette.

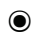

David Brême, docteur en sciences des religions (2018, UQÀM, Québec) a effectué un terrain de recherche au printemps 2013 à l'Ashram de Sri Aurobindo à Pondichéry, à Auroville et au Centre Sri Aurobindo de Montréal. Il invite le lecteur à découvrir le Centre Sri Aurobindo de Montréal en décrivant son aspect général, puis en abordant des aspects plus spécifiques concernant son hybridité culturelle indoquébécoise. Sous un aspect général, il présente une analyse reconduisant également en partie la théorisation wébérienne de l'institutionnalisation des mouvements ayant eu un fondateur charismatique suite à leur routinisation, avec la spécificité à cet égard du rôle de la fondatrice du Centre Sri Aurobindo de Montréal, Madeleine Gosselin. À cette présentation succède une partie visant à mettre l'accent sur la dimension participative de membres du Centre Sri Aurobindo. L'auteur s'appuie à cette fin sur une analyse de la réception montréalaise de l'Inde et de sa spiritualité (Zeghiche, 2011 ; Geoffrion, 2011 ; Timiri, 2009) et sur la réflexion de la notion d'hybridité culturelle pensée par Homi K. Bhabha (2007), un auteur clé des études postcoloniales. Étant donné qu'aucune étude n'a été publiée à ce jour sur ce site, sa démarche est exploratoire. 


\section{Contexte historique}

\section{du mouvement spirituel Mère-Sri Aurobindo}

David Brême

\subsection{Biographie d'Aurobindo Ghose de 1872 à 1914}

\section{Chronologie biographique de Sri Aurobindo}

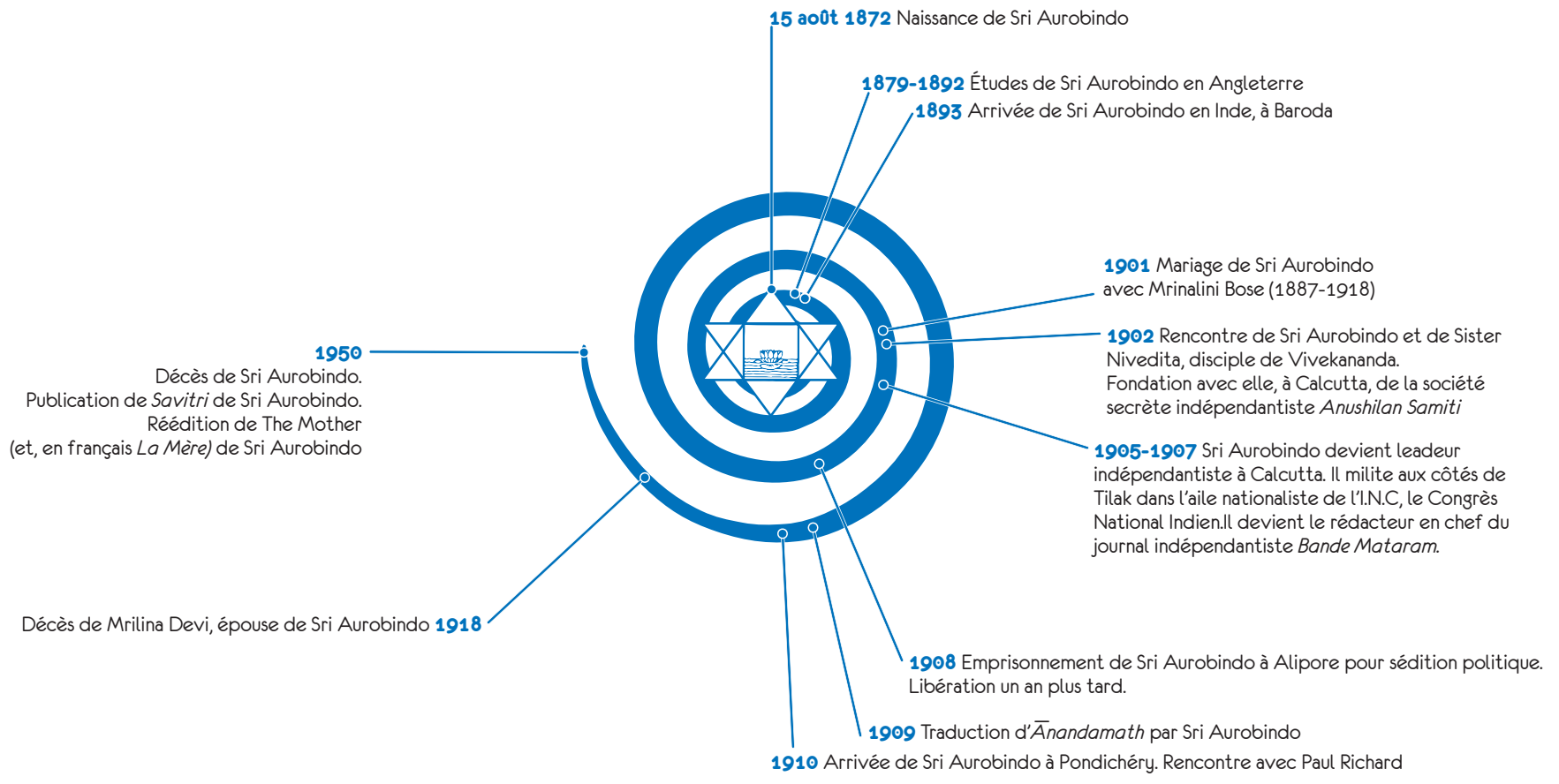

Sri Aurobindo (1872-1950), né Aravinda Ackroyd Ghose à Calcutta d'un père chirurgien fils du patriote Kaliprasad Ghose et d'une mère fille de brahmane, fut souvent appelé Aurobindo Ghose jusqu'en 1914, date de sa rencontre avec
Mirra Alfassa. Ce résumé de sa biographie de 1872 à 1914 se fonde principalement sur le travail biographique de Peter Heehs (1989/2003) et sur les présentations de Sri Aurobindo par Marie Horassius (2012) et Patrick Beldio (2016). 
À la suite de la maladie de la mère d'Aurobindo Ghose et pour de multiples raisons ${ }^{1}$, en 1879, il fut envoyé à 7 ans avec ses deux frères aînés à Manchester pour y recevoir une éducation anglaise. Son père demanda aux tuteurs anglicans de ses enfants, $M$. et $\mathrm{M}^{\mathrm{me}}$ Drewett, de ne jamais mentionner la religion et la culture indienne afin qu'ils soient mieux adaptés à la culture anglaise. Aurobindo Ghose se révéla très vite un excellent élève et le seul apte à exaucer les aspirations de son père voulant voir ses enfants entrer dans les Services administratifs indiens. À partir de 1884, à l'école Saint-Paul de Manchester, puis à partir de 1889 à l'Université Cambridge, il apprit l'anglais, le latin, le grec, le français, l'italien et même quelques notions de bengali. Il remporta une bourse d'excellence pour faire ses études classiques. Malgré toutes ses capacités, il ne souhaita pas embrasser la carrière qui lui était tracée : arraché à sa terre natale, privé de son histoire indienne, Aurobindo s'intéressait de plus en plus à ce passé caché. Il apprit alors l'histoire de son pays d'origine. Après une brillante réussite aux examens universitaires finaux, Aurobindo ne put entrer en 1892 dans l'Indian Civil Service, car il ne se présenta pas à l'examen éliminatoire d'équitation. À 21 ans, il décida de rentrer en Inde.

À son arrivée, son père décéda et Aurobindo Ghose se mit au service du Maharadja de Baroda, auprès duquel il demeura de 1893 à 1906 en exerçant différentes fonctions. Durant cette période, il étudia le sanskrit, le bengali, la littérature indienne classique et moderne, les Veda, les Upaniṣad, le Rāmāyaṇa, le Mahābhārata incluant notamment la Bhagavad-
Gìtā, qu'il commenta de façon approfondie. Il fut aussi influencé par différentes traditions hindoues telles que le shaktisme, les Tantra et le Vedānta de Ramakrishna.

Dans ces écrits, il puisa certains éléments pour engager la lutte face aux Anglais, en écrivant malgré tout beaucoup de littérature en anglais. Il fonda ensuite en 1902 avec Sister Nivédita, C.R. Das et Surendranath Tagore une société secrète révolutionnaire au Bengale, Anushilan Samiti. Il organisa aussi avec Jatindranath Mukherjee des clubs de culture physique, qu'il espérait voir devenir la base d'une armée et d'un mouvement révolutionnaire anti-britannique. Aurobindo Ghose avait alors déjà commencé à pratiquer le yoga en 1905, initialement pour l'aider à se concentrer sur sa mission révolutionnaire.

En 1905, le Vice-Roi de l'Inde britannique, Lord Curzon, décida de diviser le Bengale en deux territoires pour mieux le dominer. Arrivé à Calcutta en 1906 au milieu d'une vague d'agitation contre cette partition imposée, Aurobindo Ghose joua un rôle clé dans la lutte indépendantiste avec d'autres grandes figures telles que Lokmanya Tilak, Rabrindanath Tagore, Sister Nivedita, Jatindranath Banerjee ${ }^{2}$ et Jatindranath Mukherjee ${ }^{3}$, son bras droit (Mukherjee, 2010). Il multiplia ainsi les actions : boycottage, réunions militantes et rédaction d'écrits engagés. Il participa avec Tilak à la refondation de l'Indian National Congress (INC) au début du siècle. Il s'engagea de plus en plus intensément dans la lutte indépendantiste auprès de «Lal, Pal, Bal $»^{4}$, autrement dit les « extrémistes » de l'INC. Il devint notamment avec Bipin Chandra Pal corédacteur en chef du

\footnotetext{
${ }^{1}$ Le choix de la profession médicale fait par le père d'Aurobindo Ghose impliquait la déchéance de sa caste, car toucher les corps est impur dans la tradition hindoue. De plus, contrairement à son beau-père inscrit dans le mouvement du Brahmo-Samaj, sorte d'hindouisme monothéiste réformé sans caste, il semblerait que le père d'Aurobindo ait été très rationaliste et peu favorable à l'hindouisme, même de façon réformée.
}

2 Dit Niralamba Swami à partir de 1910

3 Dit Bagha Jatin

4 Lajpat Rai (1865-1928), Bal Gangadhar Tilak (1856-1910), Bipin Chandra Pal (1858-1932) 
journal souverainiste Bande Mataram et, finalement, son rédacteur en chef.

Il professait officiellement la poursuite du mouvement swadeshi (boycottage non violent), mais défendait aussi l'usage légitime de la violence par un peuple opprimé et cautionnait officieusement les attentats que son frère révolutionnaire Barindrakumar organisait avec son groupe de résistants contre les autorités anglaises. Arrêté en 1908 avec Barindrakumar et d'autres membres du groupe révolutionnaire suite à un attentat à la bombe, Aurobindo Ghose fut accusé d'avoir conspiré contre le gouvernement. Après un procès d'un an, il fut acquitté pour manque de preuves en mai 1909. Ses amis furent pour certains fusillés, et d'autres exilés (comme Bipin Pal au Mexique) ou déportés (comme son frère sur les îles Andamanet-Nicobar.)
Avant son arrestation et pendant son emprisonnement, Aurobindo Ghose vécut plusieurs expériences spirituelles transformatrices. À sa sortie de prison, Aurobindo s'exila avec un groupe d'une douzaine d'amis révolutionnaires dans le sud de l'Inde en 1910 à Pondichéry, qui était alors un territoire français. Durant les quatre années suivantes, il resta invisible, poursuivant son travail de rédacteur, ses recherches spirituelles (Heehs, 2008) et, de manière voilée, son activité politique subversive, notamment par l'intermédiaire de son disciple bengali Motilal résidant à Chandernagor. Il lui envoya des lettres qu'il signait Kālì et qui ont pu contenir des consignes codées pour mener des actions révolutionnaires (Stoeber, 2009 : 304).

La suite de sa biographie est indissociable de celle de son associée, la " Mère », à laquelle s'intéressera la section suivante.

\subsection{Biographie de Mirra Alfassa, dite la Mère (1878-1973), et de Sri Aurobindo de 1914 à 1950}

\section{Chronologie biographique de la Mère (Mirra Alfassa)}

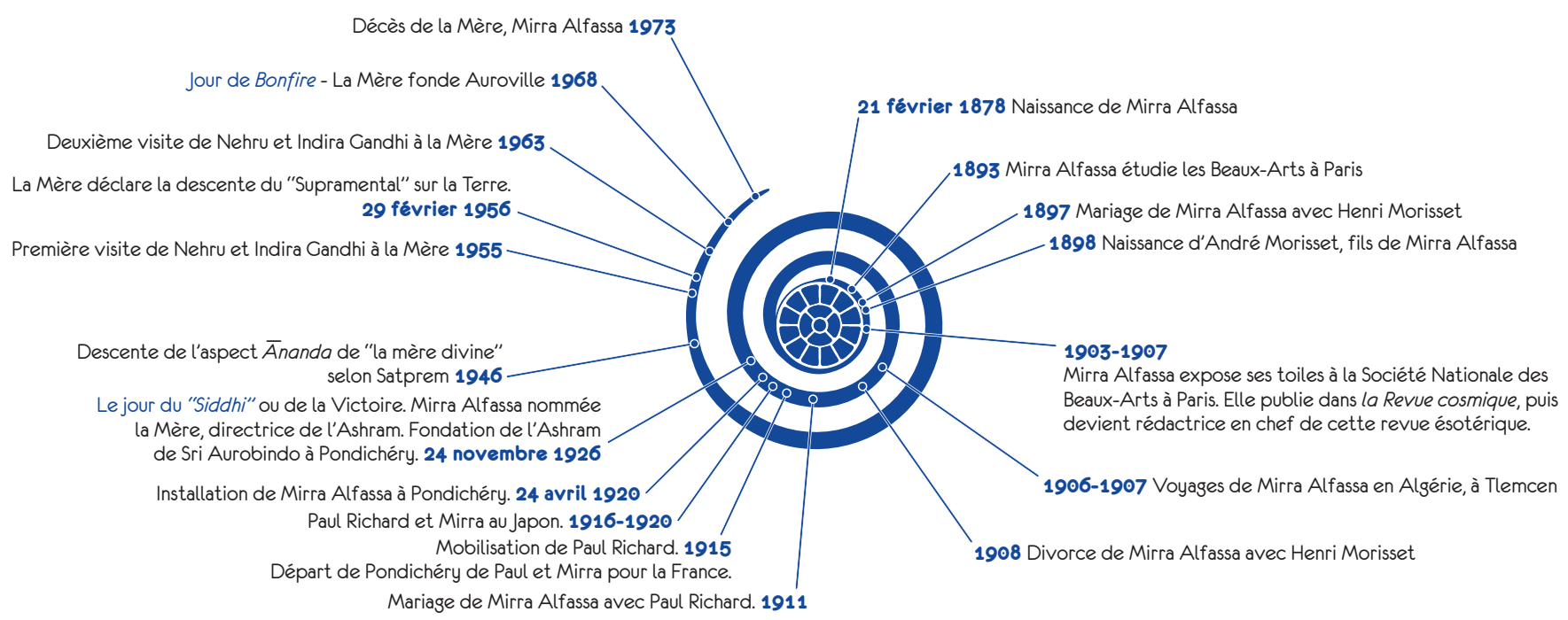

L'aperçu de la vie de Mirra Alfassa s'appuie principalement sur l'ouvrage La Mère, une biographie de Georges Van Vrekhem (2007), qui a puisé à la fois dans des fonds d'archives du
Mouvement spirituel Mère-Sri Aurobindo et dans les Archives nationales françaises. Ces informations ont été confrontées à d'autres sources hagiographiques en français et en 
anglais, notamment à l'imposant volume de K. R. Srinivasa Iyengar, On the Mother (1994), et à l'hagiographie plus concise de Wilfried (1990). Sans que ces sources puissent constituer une historiographie, elles suffisent à notre objectif de donner quelques repères biographiques. Par ailleurs, pour la période allant de 1914 à 1950, la biographie de Sri Aurobindo a été conjointe afin d'éviter des répétitions narratives.

Mirra Alfassa naquit le 21 février 1878. Installés à Paris depuis moins de deux ans, ses parents, originaires de l'Empire ottoman, étaient d'origine juive, mais ils n'auraient pas eu de pratique religieuse. Son père était banquier, ainsi que des hommes de la famille de sa mère. Sa grand-mère maternelle Mira Ismalun organisa la venue de ses parents à Paris, à l'occasion de voyages d'import-export de produits de luxe entre le Proche-Orient et Paris.

Mirra eut une enfance bourgeoise à Paris et étudia les Beaux-Arts à l'Académie Julian à partir de 1893. Peintre diplômée de cette académie, elle eut une riche vie culturelle, appréciant la musique, la sculpture et le théâtre. Elle connut Auguste Rodin et Gustave Moreau dont l'un des élèves, Henri Morisset devint son mari en 1897. Son fils André naquit en 1898. Elle tomba malade à la suite de son accouchement. André fut confié à la famille de son mari. Par la suite, elle exposa certaines de ses œuvres au Salon de la Société Nationale des Beaux-Arts de 1903 à 1905 à Paris, contribuant à l'émancipation artistique des femmes en France ${ }^{5}$.

Peu après, son centre d'intérêt se déplaça de la peinture vers l'ésotérisme. En 1903, elle fit connaissance de Louis Thémanlys (1874-1943), responsable du Mouvement Cosmique. Elle se consacra alors à la rédaction de La Revue Cosmique (Barlet et Thémanlys, 1901-1908)6, publication mensuelle parisienne, en transcrivant des articles et des contes ésotériques de Max Théon (1845-1927) et de son épouse Alma$^{7}$ (1858-1908) qui résidaient en Algérie. Elle leur rendit visite une première fois en 1906 et une dernière fois en 1907.

Elle divorça d'Henri Morisset en 1908, acte anticonformiste à l'époque. Ensuite, elle organisa des groupes de discussion autour des thèmes de l'ésotérisme et de l'éveil de la pensée, dans le cadre d'associations telles que L'Union de la Pensée féminine (La Mère, 1983, 83-102). Elle devint l'amie d'Alexandra David-Néel (2010, 94-95). Selon Georges Van Vrekhem, « Mirra, comme Alexandra David-Néel, était une féministe convaincue et active ». À cette époque les femmes n'avaient pas le droit, "sans autorisation, [de] se présenter à un examen, [de] s'inscrire dans une université ». Or, le programme proposé ${ }^{8}$ par Mirra à L'Union de la Pensée féminine visait principalement à éveiller la liberté de pensée des femmes.

Après 1908, Mirra rencontra Paul Richard, fonctionnaire au Ministère des Colonies, et l'épousa en 1911 à Paris. Paul Richard avait déjà rencontré Aurobindo à Pondichéry en 1910.

Mirra et Paul Richard partirent ensemble à Pondichéry en mars 1914, à la veille de la déclaration de la guerre. Paul Richard brigua le siège de député de l'Inde française, mais il ne fut pas élu. Mirra rencontra Sri Aurobindo le 29 mars 1914 et en témoigna ainsi :

5 «En 1800, 66 œuvres exposées au Salon, c'est-à-dire 12,2 \% du nombre total, étaient des œuvres de femmes. En 1900, les chiffres avaient sérieusement augmenté : 609 œuvres de femmes, c'est-à-dire 21,2 \% du total.» (Higonnet 1991, 319)

6 Revue consultable et consultée sur microfilms à la Bibliothèque Nationale Française.

7 Leurs noms d'état civil sont Louis Maximilien Bimstein et Mary Christine Woodroffe Maurel.

8 Certains de ses discours et de ses comptes rendus de réunions furent publiés par la suite en 1955 dans le livre Paroles d'autrefois (La Mère, 1983). 
« Avant même de venir, de partir pour rencontrer Sri Aurobindo, j'avais réalisé tout ce qu'il fallait réaliser pour pouvoir commencer son yoga. C'était tout prêt et classé, organisé - magnifique ! Avec une superbe construction mentale! Qu'en cinq minutes, il a jetée par terre. Oh ! Comme j'étais heureuse ! Ouf !... Ça, ça a été vraiment la récompense de tous mes efforts. [...] Je me suis assise à côté de lui, simplement, comme cela, par terre [...] [,] tout d'un coup, j'ai senti en moi comme une grande Force - une paix ! Un silence ! Massif. [...] Et puis je me suis aperçue que je n'avais plus une pensée [...], que j'étais absolument dans un blanc complet. Alors j'ai rendu grâce au Seigneur et j'ai remercié Sri Aurobindo dans mon cœur. » (Satprem, 1979b, 305-306)

Cette rencontre changea complètement le cours de sa vie. Ce fut également à cette occasion qu'Aurobindo Ghose prit le nom de Sri Aurobindo (Van Vrekhem, 2007 : 157). Sri Aurobindo, Paul Richard et Mirra éditèrent après la revue $A r y a^{9}$, traduite en français par Mirra. Ce fut dans cette revue que les premières œuvres majeures de Sri Aurobindo furent publiées par épisode, ce qui constitue aujourd'hui plus de 30 livres dans les domaines du yoga, de la politique, de la culture, de l'art et de la poésie.

Quelques mois après l'arrivée de Mirra et Paul Richard à Pondichéry, la Première Guerre mondiale éclata. Paul Richard fut mobilisé six mois après et sommé de rejoindre la métropole : il retourna à Paris avec Mirra le 22 février 1915. En mars 1916, Paul Richard fut mandaté par le ministre des colonies pour effectuer une mission commerciale au Japon. Les époux Richard débarquèrent le 18 mai 1916 au Japon et $y$ restèrent jusqu'en 1920. Là-bas, Mirra rencontra
Rabindranath Tagore, découvrit la philosophie bouddhiste zen et le shintoïsme.

Pendant ce temps, en Inde, le 17 décembre 1918, Mrinalini Devi Ghose, épouse de Sri Aurobindo, décéda à Calcutta d'une grippe foudroyante, alors qu'elle était en chemin pour le rejoindre à Pondichéry.

Mirra Alfassa et son mari quittèrent le Japon et arrivèrent à Pondichéry le 24 avril 1920. Mirra Alfassa y demeura jusqu'à la fin de ses jours. Selon Georges Van Vrekhem, la " collaboration décisive entre Mirra et Sri Aurobindo » $(2007,215)$ fut insupportable pour Paul Richard et l'aurait rendu violent10. Il quitta Mirra en novembre 1920. Deux ans après, il lui demanda le divorce : elle y consentit aussitôt. La résidence communautaire du 41 rue François Martin, abritant Sri Aurobindo, la Mère et leurs disciples devint le cœur de l'ashram et est aujourd'hui l'adresse du Samadhi, lieu d'inhumation des fondateurs et espace de méditation.

Le 24 novembre 1926, Sri Aurobindo reconnut publiquement Mirra Alfassa comme étant "la Mère » et sa Śakti. Lui-même se « retira » dans sa chambre en 1926 pour parfaire son yoga, sauf à l'occasion de darshan publics où il donnait sa bénédiction par le regard à ses disciples assemblés, avec lesquels il entretint par ailleurs une correspondance très nourrie. La position conférée par Sri Aurobindo à Mirra suscita des oppositions de certains disciples, mais finit par être acceptée avec son soutien. En 1937, la Mère entreprit la construction d'un bâtiment aux qualités architecturales remarquables, décrites par Patrick Beldio dans sa thèse (2016). Le 24 novembre 1938, Sri Aurobindo se brisa une jambe en allant dans sa chambre et rompit partiellement son isolement pour être aidé par certains de ses disciples.

\footnotetext{
9 Un article de cette revue publié dans le troisième numéro, en octobre 1914, commente la signification de ce titre « Arya » qui désignerait notamment, à son stade ultime, l'Arhat, l'être conscient par excellence.

10 «Ses éclats de frustration et de colère devenaient si violents qu'il lui arriva de jeter le mobilier par la fenêtre ou de prendre Mirra à la gorge pour l'étrangler ». (Van Vrekhem, 2007 : 215)
} 
Lorsque la Deuxième Guerre mondiale éclata, Sri Aurobindo prit officiellement le parti des Alliés, en déposant symboliquement une contribution monétaire à la Couronne d'Angleterre. Contrairement à des Indiens qui voyaient dans une alliance avec l'Allemagne une occasion de se défaire du joug britannique, Sri Aurobindo était conscient du danger encore plus grand que constituait le nazisme. Aussi, il s'engagea fermement aux côtés de l'ennemi colonial d'hier. Le 11 novembre 1941, il envoya une dépêche au Congrès National Indien pour leur enjoindre d'accepter la proposition britannique d'établir l'Inde en dominion, qui lui apparaissait la solution la plus efficace pour obtenir l'émancipation de l'Inde ; cela fut refusé par le Congrès, Nehru et Gandhi.

En 1943, la Mère fonda une école pour les enfants réfugiés à l'Ashram en raison de la guerre et du danger qui guettait le nord de l'Inde. En 1947, l'Inde devint indépendante. Sri Aurobindo décéda en 1950 et son corps demeura exposé trois jours à Pondichéry sans souffrir de traces apparentes de décomposition. Savitri, son œuvre poétique majeure rédigée pendant plus de 35 ans, publiée peu après son décès, peut être considérée comme son testament spirituel. On lui doit aussi la fameuse phrase d'investiture de Nehru affirmant l'importance de construire une Inde unie, mais une « unité dans la diversité ».

La Mère continua à diriger l'Ashram. En 1952 fut fondé le Centre International d'Éducation Sri Aurobindo.

En novembre 1949, la Mère reçut la visite de son fils André qui devint secrétaire de l'Ashram après le décès de Pavitra, un disciple qui lui était proche.

En 1954, un accord franco-indien fut signé pour le rattachement de l'Inde française à l'Inde indépendante. À la suite de cet accord, en septembre 1955, la Mère reçut Nehru et sa fille Indira Gandhi à l'Ashram de Sri Aurobindo. Le 13 juin 1963, après le rattachement officiel de l'Inde française, la Mère les reçut pour la seconde et dernière fois ${ }^{11}$.

Par la suite, en 1968, la Mère fonda la communauté internationale d'Auroville dans le Tamil Nadu, sur un terrain aride à dix kilomètres au nord de Pondichéry. Auroville est un lieu dont la vocation est la construction d'une cité internationale avec des idéaux de paix, d'unité humaine et de réalisation de la vision de la Mère à ce sujet. Sous la supervision de la Mère, l'architecte français Roger Anger (1923-2008) en conçut les plans : la maquette, toujours visible dans le bâtiment administratif de la ville, a la forme d'une galaxie organisée autour d'une architecture sphérique centrale, le Matrimandir. La Mère décéda en 1973, lors du dernier bétonnage des quatre piliers du Matrimandir.

11 On peut souligner que le rattachement officiel de Pondichéry à l'Inde eut lieu en août 1962, après que Charles de Gaulle ait signé les accords d'Evian qui ont rendu l'Algérie indépendante. Le laps de temps de décolonisation entre l'indépendance de l'Inde en 1947 et le rattachement de Pondichéry en 1962 est dû en grande partie au refus français de « faire de l'Inde » un exemple incitatif pour leurs autres colonies, dont l'Indochine et l'Algérie. 


\section{Le yoga artistique de la Mère à l'Ashram de Sri Aurobindo Patrick Beldio}

\subsection{L'Ashram de Sri Aurobindo à Pondichéry}

Rétrospectivement, la fondation de l'Ashram de Sri Aurobindo peut être reliée à une expérience qui remonterait au 24 novembre 1926 : Sri Aurobindo affirme avoir expérimenté alors une nouvelle union avec l'énergie divine, qu'il identifia comme Krishna et qu'il nomma ultérieurement le «surmental " ( The Overmind »). Cette date est appelée aujourd'hui "le jour du Siddhi » (la victoire). Cette expérience spirituelle fut interprétée comme l'indicateur d'un haut degré de victoire (Siddhi), assurant le but ultime de " ramener ici-bas le supramental », c'est-à-dire de diviniser définitivement la matière (Sri Aurobindo, 2011 : 346-49).

L'Ashram a été conçu comme un laboratoire par la Mère et Sri Aurobindo afin de contenir la nouvelle énergie du surmental et de préparer la future transformation supramentale de la matière ${ }^{1}$. Les modèles traditionnels de la vie ashramique, tels que la méditation et la contemplation, seraient désormais orientés autour d'un service pragmatique et désintéressé dans le monde physique, exprimé par la maxime de Sri Aurobindo : « Toute la vie est un yoga » (Sri Aurobindo, 1999). La Mère écrivit ainsi :

«Chacun doit consacrer au moins un tiers de son temps à un travail utile. Toutes les activités sont représentées à l'ashram et chacun choisit le travail le plus conforme à son tempérament, mais il doit le faire dans un esprit de service et de désintéressement, en gardant toujours présent à l'esprit le but de la transformation intégrale. Pour que cela soit possible, l'ashram est organisé de telle façon que les besoins raisonnables de tous les sâdhaks se trouvent satisfaits et qu'ils n'aient pas à se soucier de leur subsistance. Il y a très peu de règlements pour que chacun puisse jouir de la liberté nécessaire à son développement, mais certaines choses sont strictement interdites : (1) la politique, (2) le tabac, (3) l'alcool, (4) le plaisir sexuel. Une attention particulière est apportée à la bonne santé, au bien-être et à la croissance normale du corps de tous, petits et grands, jeunes et vieux. » (La Mère, 2009a : 112)

Ce mode de vie avait en fait été un de ses rêves dès sa jeunesse en France. Elle expliqua une fois à un étudiant :

«Je m'étais toujours dit que, si jamais je pouvais le faire, je tâcherais de créer un petit monde. Oh ! Un tout petit, mais enfin... un petit monde où les gens

\footnotetext{
1 En fait, le 29 février 1956, la Mère expérimenta cette manifestation supramentale après le décès de Sri Aurobindo, ce qui est maintenant célébré sous le nom de «The Golden Day », l'or étant ce que ces guru décrivent comme la nature et la couleur de la conscience supramentale.
} 
pourraient vivre sans avoir à se préoccuper de la nourriture, du logement, du vêtement et des nécessités impérieuses de la vie, afin de voir si toutes les énergies, libérées par cette certitude de l'existence matérielle assurée, se tourneraient spontanément vers la vie divine et vers la réalisation intérieure. » (La Mère, 1968 : 181)

Les deux guru dirigèrent de différentes manières l'« énergie libre " de l'Ashram en l'orientant vers des activités telles que des services de publication, une école, un gymnase, etc. Le mode de direction de l'Ashram était inhabituel au sens où Sri Aurobindo se retira dans sa chambre en 1926, pour se concentrer sur son travail de transformation de la matière jusqu'à sa mort en 1950. Ses étudiants le voyaient en de rares occasions, appelées les jours de darshan. Lui et la Mère s'asseyaient alors ensemble dans ses appartements tandis que des centaines de personnes à la file indienne passaient avec révérence pour recevoir leurs bénédictions par le "regard sacré »(darśana). C'était un échange silencieux d'amour et de sagesse entre dévots et guru. Une fois le darshan terminé, Sri Aurobindo se retirait dans ses appartements. Bien qu'ils puissent être considérés comme opposés - car l'un était retiré dans sa chambre et l'autre était très active dans l'Ashram - Sri Aurobindo et la Mère se complétaient l'un l'autre. Par exemple, quand un étudiant avait besoin d'un conseil ou d'un échange formel, ils écrivaient à la Mère et Sri Aurobindo leur répondait. Cela permettait de maintenir les deux guru dans l'imaginaire collectif comme le " guru unique » représentant la totalité sacrée androgyne.

En 1928, l'Ashram comptait environ soixante-dix membres. En 1937, sa population atteignit environ deux cents membres. Au début, c'était une communauté de Bengalis et de Goujaratis avec plusieurs Européens et Américains, surtout des hommes : les femmes avaient des rôles domestiques déterminés par la société indienne, ce qui rendait difficile, sinon impossible, de quitter leur foyer pour rejoindre un ashram. Cependant, cette structure sociale fut transformée par la Deuxième Guerre mondiale. La population de l'Ashram augmenta jusqu'à 400 membres dans les années 1940, dont de nombreuses femmes ; certaines amenèrent leurs enfants, fuyant la menace potentielle d'une invasion par le Japon. La Mère n'avait pas permis aux enfants de vivre à l'Ashram, car sa discipline spirituelle n'était pas conçue pour eux ; toutefois, avec cette crise de réfugiés, elle leur ouvrit les portes et établit une école pour eux le 2 décembre 1943. Ce fut seulement en 1952, après le décès de Sri Aurobindo, qu'elle élargit l'école et inaugura le Centre Universitaire International Sri Aurobindo. Elle changea ultérieurement son nom en 1959 pour l'appeler le Centre d'Éducation International Sri Aurobindo, qui continue d'éduquer des élèves de la maternelle jusqu'au niveau du lycée.

En 1945, la Mère fonda le Département d'éducation physique au sein de l'école de l'Ashram pour encourager l'exercice physique, afin de renforcer le corps pour la manifestation supramentale. En guise de célébration pour la fondation de l'école de l'Ashram, elle institua une manifestation sportive annuelle intergénérationnelle le 2 décembre avec des démonstrations de la maîtrise d'un sport ou d'un exercice particulier sur le terrain de sport de l'Ashram. Les équipements en éducation physique et sportive incluent " une piste de course cendrée de 4 lignes, des terrains et des courts pour le football, cricket, hockey, volleyball, basketball et tennis, une piscine et un gymnase pour la gymnastique et le culturisme. Il y a aussi des équipements pour la boxe, la lutte indienne, le judo et le roller $»^{2}$.

Dans les années 50, la Mère enseignait, jouait au tennis, dirigeait le travail dans tous les secteurs et, en tant que guru, développait des relations de confiance avec ses étudiants. Elle était particulièrement douée en tant

2 http://sriaurobindoashram.org/ashram/saice/physical.php, consulté le 20 septembre 2017. 
qu'administratrice. Elle s'assurait de pourvoir la nourriture, les vêtements et le logis comme base d'apprentissage au niveau émotionnel, psychologique, intellectuel et spirituel. Elle fit construire des jardins pour cultiver légumes et fleurs, des fermes d'élevage de volaille et de bétail, des greniers, des cuisines. Elle créa aussi un atelier de céramique pour la vaisselle et des briques de construction, des ateliers de confection de vêtements, de toiles pour l'art, une fabrique de papier et une laverie. Il existe un magasin d'équipements pour travailler le bois, une fonderie et des secteurs pour la construction et l'architecture des bâtiments, conçus pour maintenir leurs infrastructures. En plus des bibliothèques et de l'école, on trouve encore un studio de photographie, de vidéographie et une importante imprimerie pour les textes de la Mère et Sri Aurobindo, édités en plusieurs langues. Un théâtre est utilisé pour des programmations musicales, d'art dramatique et de danse, célébrant les principes spirituels de l'Ashram.

En 1962, la Mère tomba gravement malade et se retira dans ses appartements pour le reste de sa vie. De ce modeste logement, elle conçut une grande idée. À 90 ans, en 1968, elle inaugura Auroville, à environ $12 \mathrm{~km}$ de l'Ashram. C'est une entité distincte, avec un but différent de celui de l'Ashram, bien qu'ils soient souvent confondus.
Tandis qu'Auroville est une expérimentation organisée souplement et cherchant à mettre en pratique certains principes spirituels d'unité humaine que les membres peuvent attribuer ou non aux guru, l'Ashram est un lieu pour le discipulat spirituel de dévots qui se soumettent à une vie très disciplinée que la Mère avait établie, afin de pouvoir devenir ce qu'ils appellent « une communauté gnostique "d' "êtres supramentaux »: le but est une transformation harmonieuse, tout d'abord de la conscience, puis du psychisme, jusqu'à éventuellement pouvoir transformer par leur yoga le corps, de telle sorte qu'il dépasse la maladie, la souffrance et la mort, à l'instar de la quête et de l'endurance de leurs guru.

Lorsque la Mère mourut en 1973, elle ne désigna personne pour prendre sa succession. L'Ashram est administré par un Trust, créé en 1955 par la Mère afin de garantir son avenir. Le Trust ne tient pas un compte détaillé du nombre de dévots ; toutefois, en 2017, Devdip Ganguli, membre du Trust, m'a indiqué ${ }^{3}$ qu'il y avait environ 1200 membres à temps plein et entre 3000 et 4000 personnes informellement associées à l'Ashram à Pondichéry. Les femmes représentent plus de la moitié de ce nombre. Environ $12 \%$ des personnes sont de nationalité étrangère.

3 Devdip Ganguli du Sri Aurobindo Ashram Trust, courriel à l'auteur, 30 août 2017. 


\subsection{Le yoga artistique de la Mère à l'Ashram de Sri Aurobindo}

Peintre, la Mère ne recherchait pas le succès commercial ou mondain de ses œuvres, mais peignait, selon ses écrits, pour être en contact avec son âme et exprimer sa vie spirituelle intérieure. Elle continua d'utiliser ce processus créatif dans cette direction, y compris en coopérant avec des étudiants de l'Ashram après qu'elle soit allée en Inde et soit devenue une guru.

\subsubsection{La relation des disciples avec la « guru »}

Afin de comprendre le travail de la Mère dans son art et son yoga, il est utile de définir le mot guru, qui renvoie à une fonction au sein de l'hindouisme, que la Mère endossa en partie. Dans la tradition hindoue de la relation entre maître et étudiant, Karen Pechilis écrit que le « guru est entendu comme expérimentant le réel continuellement », le " réel » étant l'expérience de la réalité ultime ou Brahman, qui est aussi nommé " Être - Conscience - Béatitude » ou Sat-Cit-Ānanda »(Pechilis, 2004 : 4)4. De plus, le " guru est à même d'inspirer l'expérience du réel aux autres dans la finalité de leur progression spirituelle, de l'auto-réalisation totale ou de leur évolution en tant qu'être humain » (2004: 5). Létymologie du mot guru puise ses racines sanscrites soit dans le radical gri, voulant dire invoquer ou prier, soit dans le radical gur, signifiant s'élever, faire un effort. En tant qu'adjectif, guru a pour sens " pesant ", ce qui sous-entend " qui a son poids de sagesse » ou « son poids de signification spirituelle » (MonierWilliams, 2005) ${ }^{5}$. Guru est un terme de la même famille que le terme latin gravis qui signifie grave, pesant ou sérieux ${ }^{6}$. Le guru pourrait alors signifier le poids de la force persuasive de la sagesse, stimulant l'élévation de la conscience de l'étudiant.
Quelle est la relation de la Mère, en tant que guru spirituelle, avec l'art et le processus créatif ? Dans la compréhension de Sri Aurobindo, l'art ancien sacré de l'Inde exprime un idéal "d'un corps subtil et divin » : la manière adéquate de réaliser cet idéal est de recourir à l'aide d'un saint ou sādhu, dans la mesure où ce dernier l'incarne de façon vivante (Sri Aurobindo, 1997a : 296). C'est pourquoi, concernant l'art, le but serait de créer un art qui satisfasse le goût du saint, ou du saint en nous, lequel rechercherait la perfection ou la parfaite expression de soi. La Mère instruisit certains de ses étudiants par les arts pour accroître leur perfection potentielle : dans ce processus, ils se sont reliés à elle comme le modèle de sainteté et de perfection auquel leur art et leur croissance pouvaient être mesurés. Pour ses étudiants, la Mère a « l'âme la plus profonde et l'imagination psychique la plus sensible ", car elle manifestait la Mère divine sous forme humaine (Sri Aurobindo, 1997a : 296). Face à sa stature extraordinaire, ils se reliaient à elle pour guider leur créativité, afin de progresser spirituellement d'une façon plus rapide et plus dépendante que s'ils avaient suivi leur propre direction.

Lorsque nous étudions les interactions des disciples avec la Mère, nous observons qu'un conflit les traverse entre la confiance qu'ils lui

\footnotetext{
4 Traduction française de Karen Pechilis (2004).

5 Traduction française du dictionnaire Monier-Williams (2005), article «guru».

6 Traduction française de Robert K. Barnhart (1998: 447).
} 
accordent et leur besoin de tester son autorité. Cependant, le but de ses étudiants artistes est de rejeter ce qui est bas, d'aspirer à ce qui est élevé et de s'en remettre à la nature intérieure ultime. La Mère représente cette nature intérieure ultime aux yeux de ses disciples : ainsi ils recherchent à intérioriser sa guidance, tout en extériorisant le meilleur de leur aspiration, malgré ou grâce à leur conflit interne. La Mère enseignait à ses étudiants que «l'opposition et les contraires sont des stimulants pour le progrès ", dans la mesure où, selon elle, l'opposition « est le moyen le plus rapide et le plus efficace pour faire sortir la création de son inertie et la faire avancer jusqu'à son épanouissement »(La Mère, 2009b : 204). Ainsi, plutôt que d'éviter le conflit, la Mère encourageait ses étudiants à l'affronter et à l'utiliser pour progresser dans la croissance de leur conscience.

\subsubsection{La peintre Huta, disciple de la Mère}

Cette dynamique peut être observée à travers la collaboration entre la Mère et ses étudiants artistes. Quoiqu'elle ait rarement peint après son arrivée à Pondichéry en 1920, elle dessina avec des peintres jusqu'en 1972, un an avant son décès. Pendant 52 ans, elle aspira à utiliser la peinture de la même manière que Sri Aurobindo utilisait la poésie : pour perfectionner la conscience et voir jusqu'où ce processus de croissance pourrait affiner et perfectionner l'art lui-même. Sri Aurobindo écrit au sujet du processus créatif dans la composition de son poème épique Savitri : «En fait, Savitri n’a pas été pensé par moi comme un poème qui doit être écrit et achevé, mais plutôt comme un champ d'expérimentation pour voir jusqu'où la poésie peut-elle être écrite dans l'élévation de la conscience yogique et dans la créativité » (Sri
Aurobindo, $1995:$ :728)7. De la même manière que Sri Aurobindo expérimentait «La Poésie du futur " (Sri Aurobindo, 1997b) dans Savitri, la Mère voulait « exprimer la Lumière Divine sans ombre dans la Peinture du Futur " (Huta, $2001: 8)^{8}$.

La fonction de guru de la Mère et son administration des activités de l'Ashram ne lui laissaient pas assez de temps pour ce projet. Elle confia à sa collaboratrice Savita « Huta " Hindocha9 (1931-2011), artiste peintre, qu'« ici, à l'Ashram, j'encourageais différentes personnes [à peindre la "peinture du futur"] avant que tu ne sois née, mais [je n'avais] pas de disponibilités. Maintenant tu combles tous mes souhaits. Comme physiquement je n'ai pas le temps, j'utiliserai seulement tes mains. Essentiellement,

7 Traduction française de Sri Aurobindo (1995 : 728).

8 Traduction française de Huta.

9 « Huta ", un nom que la Mère lui donna, signifie « celle qui est offerte ", venait d'une riche famille indienne vaishnave au Kenya, qui possédait de nombreux moulins à sucre. Jeune, elle voyagea dans de nombreuses régions du monde, notamment en Europe, au Moyen-Orient et en Asie. Finalement, sa famille arrangea son mariage avec un homme à Bombay, contre la volonté de Huta de rester célibataire.

Bien qu'elle se fût mariée, Huta décida de quitter son mari et de rejoindre l'Ashram de Sri Aurobindo. Quand elle rencontra la Mère en 1954 pour la première fois, cela lui laissa une impression durable. Sans le faire savoir, Huta quitta définitivement sa maison quelques mois plus tard, en 1955, à l'âge de vingt-trois ans. Sa famille la poursuivit dans le but de la réunir avec son mari, mais Huta resta ferme. Finalement, la famille soutint sa décision et lui envoya même de l'argent pour commencer une nouvelle vie là-bas, car beaucoup d'entre eux reconnurent le statut spirituel de la Mère. Son mari lui-même accepta de divorcer. 
la création entière sera la mienne. » (Huta, 2009: 96) ${ }^{10}$ Cette " création entière " par la Mère devint une très imposante série de 474 peintures à l'huile intitulées Meditations on Savitri. Elle dévoila à Huta l'objectif de ce projet pictural : «Oui, nous allons vers une peinture qui sera à même d'exprimer la vérité supramentale des choses. » (Huta, 2009 : 96). Huta décrivit ainsi sa collaboration avec la Mère : « Mais, pour dire vrai, la peinture de tout Savitri est la création propre de la Mère, fondée non seulement sur sa série de visions, mais aussi avec sa propre guidance des dessins : ils sont un reflet de son propre yoga. » (Huta, 2001 : 12).

La relation avec l'artiste révèle la pédagogie de la Mère.

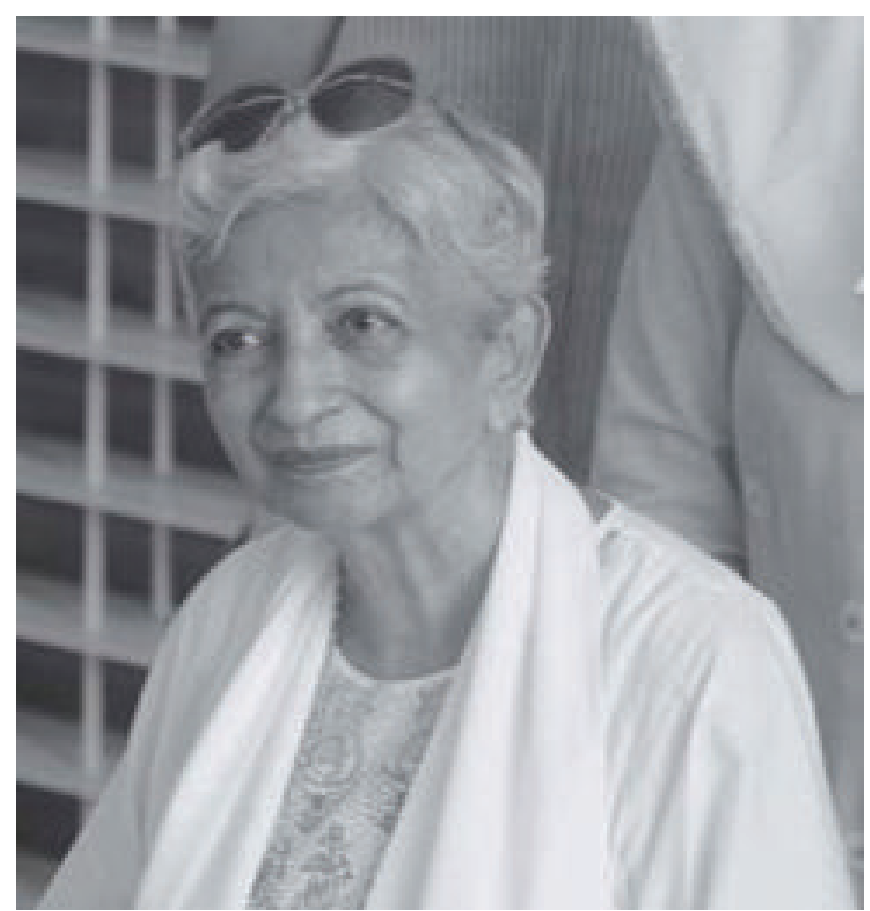

Figure 1 - Une photographie de Huta (source : https://overmanfoundation.wordpress.com/ 2011/12/06/huta-the-offered-one-a-very-specialchild-of-the-mother-by-shraddhavan/)
Pour réaliser son projet, elle élabora un entraînement à la fois spirituel et créatif pour Huta. À ce moment-là, Huta avait des problèmes psychologiques qui s'exprimaient par une hostilité envers tout le monde. La Mère la sermonna un soir à ce sujet : « Je t'aimerai si tu aimes toute l'humanité et reviens sur terre. Comprends-tu ce que je veux dire ? " (Huta, 2001 : 62) Huta s'enfuit sans répondre et écrivit : " Maintenant, j'ai commencé à haïr la Mère. » (2001 : 62) Afin de corriger ces travers, la Mère lui conseilla plus tard de développer la vertu de la générosité. Elle donna à Huta un bouquet de fleurs balsamiques ${ }^{11}$, que la Mère appela simplement « Générosité ».
Comme leur relation s'approfondissait, la Mère saisit de plus en plus d'occasions pour " gronder» Huta, selon ses propres mots. La Mère lui assura qu'" une chose dont tu peux être sûre, si je n'étais pas certaine que tu atteignes ton but, je ne te gronderais jamais, car cela serait inutile. Le fait que je le fasse est une preuve de ma conviction que tu $y$ parviendras » (Huta, 2001 : 46). Sans en avoir initialement conscience, Huta comprit probablement que ces remontrances étaient une stimulation pour sa croissance. Elle écrit : " La Mère parle ou écrit de façon plus directe ou plus sévèrement à ceux qu'elle souhaite propulser rapidement sur le chemin, car ils en sont capables, et ils ne protestent ni ne souffrent, mais sont heureux de la pression et du caractère direct, parce qu'ils savent par expérience que cela les aide à voir les obstacles et à changer » (Huta, $2001: 46)$.

Le 4 décembre 1956, l'année du jour dit «Golden Day » ou descente du supramental sur la terre, la Mère commença à préparer Huta pour son travail sur les peintures des scènes du poème Savitri de Sri Aurobindo, d'abord en lui montrant comment peindre des fleurs avec des huiles. Huta se montra réceptive, même sans formation picturale. Cela permit à la Mère de la former non seulement aux techniques de peinture, mais aussi à être attentive à ce qu'elle

10 Traduction française de Huta.

11 De la famille des Balsaminacea dont font partie les impatientes. 
appelait « la croissance de la conscience ». Elle dit à Huta :

« Toute la beauté et le charme [créés en peignant] dépendent de comment tu développes ta conscience. Avec la croissance de la conscience, les mains et les yeux deviennent aiguisés et habiles, ils reconnaissent exactement ce qui peut être fait en peinture à l'huile. Automatiquement et spontanément la chose prend forme et devient vivante et pleine de vibrations rayonnantes... Tu apprendras à peindre selon ma volonté et ma vision. » (Huta, 2009 : 210)

Pour la Mère, non seulement « l'être intérieur » doit croître en conscience, mais aussi "l'être extérieur » (le corps). Elle écrit que la croissance de chacun peut diverger légèrement dans la vie ordinaire, mais que, dans le yoga, un déséquilibre peut devenir très intense, étant donné que l'être intérieur a tendance à se mouvoir rapidement tandis que le corps risque la maladie ou même la mort. Par conséquent, entraîner le corps afin de porter autant de conscience que l'être intérieur devient très important (La Mère, 2008 : 97-101). La Mère commença la formation de Huta par ces mots : "Maintenant, méditons tranquillement sur le corps physique qui est à transformer par la lumière supramentale. » (Huta, 2009 : 211) La Mère avait elle-même appris que les mains devenaient plus conscientes à travers son propre apprentissage de la peinture et de la musique : "Il est impossible d'apprendre le piano ou la peinture sans que la conscience entre dans les mains et que les mains deviennent conscientes indépendamment de la tête. La tête peut être occupée à autre chose, cela n'a pas d'importance. » (Huta, 2009 : 211)

Afin d'éviter de bloquer la présence de la conscience dans le corps, la Mère dit à Huta :

« Dans la peinture du futur, vous ne devez pas copier aveuglément l'apparence extérieure sans la vision intérieure. $\mathrm{Ne}$ laissez jamais les idées des gens influencer votre esprit et imposer leur conseil au sujet de la peinture du futur. N'essayez pas d'adopter des techniques soit de l'art moderne soit du vieil art classique. Mais essayez toujours d'exprimer la vraie vision intérieure de votre âme et ses profondes impressions derrière les apparences pour amener la Vérité Éternelle et pour exprimer la gloire des Mondes Élevés. » (Huta, 2001 : 8-9)

Pour la Mère, le divin ne se limitait à aucune période de l'histoire de l'art ou de la culture en termes d'expression. Ultérieurement, la Mère lui dit :

" La Vérité est derrière toute chose. Le Divin demeure dans les fleurs, les arbres, les oiseaux et les rivières aussi bien que dans les êtres humains - en fait, dans toute création de la Nature. » (Huta, 2001:9)

La Mère décrit les couleurs qui caractérisent la "peinture du futur »: elles renvoient aux couleurs de l'aube qui vont du blanc transparent à l'or jusqu'au rose pâle doré. À propos de ces couleurs, la Mère dit à Huta en 1956:

"Je pense exprimer [les Mondes Supérieurs] en peignant par des couleurs variées - bleu, or, rose et blanc - avec certaines vibrations de lumière, le tout en harmonie formant le Nouveau Monde. Je souhaite amener sur la terre ce Nouveau Monde. Comme je n'ai physiquement pas le temps, je peindrai à travers toi. » (Huta, 2001: 13)

Plus tard, en 1970, la Mère dit que la descente de ce Nouveau Monde était finalement survenue sérieusement. Elle indiqua qu'elle ne voyait pas seulement ses couleurs, mais aussi ses formes, qu'elle décrit dans des termes de mode. Elle écrit : «c'est la Nature MATÉRIELLE [...] et elle a dit: "J'ai mis la robe, j'ai mis TA robe - j'ai mis ta robe pour te dire que je l'avais adoptée." Cela veut dire que la Nature matérielle a adopté 
la nouvelle création » (Satprem, 1981b : 201). Il semblerait qu'Huta ait participé à cette expérience de la Mère en 1956 : le monde physique adopta la « robe » de la nouvelle création sur le mode des couleurs de l'aube.

\subsubsection{Le «Supramental», le Nouveau Monde en couleurs vu par la Mère}

Il existe certaines conventions logiques relatives à la vision intérieure colorée du Nouveau Monde ou création par la Mère. Elle décrit le monde supramental comme des mélanges d'or de plus en plus clairs. "Au-delà » de ce monde, le domaine de Brahman est de plus en plus blanc et finalement transparent comme un diamant en son cœur. Quand le domaine du supramental se combine au monde physique qu'elle décrit comme symbolisé par le rouge -, il en résulte une sorte de couleur bronze, orange doré ou rose doré, ce qui serait en effet le cas si quelqu'un mélange des pigments or, blanc et rouge, selon leur intensité. En décrivant à Huta le type de peinture qu'elle lui demandait de réaliser, la Mère entraînait son regard à apprécier ces couleurs, le blanc étant le plus important, et à créer avec des pastels, des mélanges avec les autres teintes : " je veux que tu fasses quelque chose de nouveau. Tu dois essayer de faire la Peinture du Futur dans une Nouvelle Lumière. Il y a une raison pour laquelle je te demande de peindre surtout sur un arrière-fond blanc. C'est une tentative d'exprimer la lumière divine sans ombre dans la Peinture du Futur» (Huta, 2001 : 8) ${ }^{12}$. Ce « sans ombre » pourrait signifier que la source de lumière n'est pas extérieure, mais intérieure, de telle sorte que les formes de la nouvelle création sont lumineuses par ellesmêmes.

La Mère montra à Huta des reproductions de peintures célèbres présentant quelques-unes de ces caractéristiques. Ainsi, elle envoyait souvent à Huta des cartes reproduisant les derniers travaux artistiques du peintre romantique britannique J. M. W. Turner (1775-1851), connu comme le " peintre de la lumière $»^{13}$. Anticipant l'atmosphère des innovations impressionnistes du XIXe siècle et même les évocations émotionnelles par la couleur des artistes expressionnistes du XXe, son travail devint de moins en moins une description de la nature, mais plus directement une célébration de la couleur et de la lumière, comme s'il était ivre des interactions entre la nature et le soleil.

Après environ cinq ans de formation de plus en plus intense (1956-61), incluant une lecture approfondie "intellectuelle et esthétique » de Savitri avec deux experts de la communauté, Huta réalisa finalement sous la supervision de la Mère une série de 474 peintures intitulée Méditation sur Savitri. La Mère publia beaucoup de photos de ces travaux artistiques dans quatre livres entre 1962 et 1965. Elle pensait ces séries picturales comme des expérimentations, non comme des produits finis, car Huta était encore en train d'apprendre son métier. À ce moment, la Mère dit à Huta : «Je ne t'ai pas laissée retoucher et refaire les peintures dans ces volumes, car je voulais montrer au monde comment la conscience se développe»(Huta, 2009: 93) ${ }^{14}$.

Un an plus tard, la Mère lui demanda de refaire entièrement des peintures de 1966 et 1967, en préparation d'une exposition pour

12 Traduction française de Huta.

13 http://www.nationalgallery.org.uk/artists/joseph-mallord-william-turner; consulté le 3 mars 2016.

14 Traduction française de Huta. 


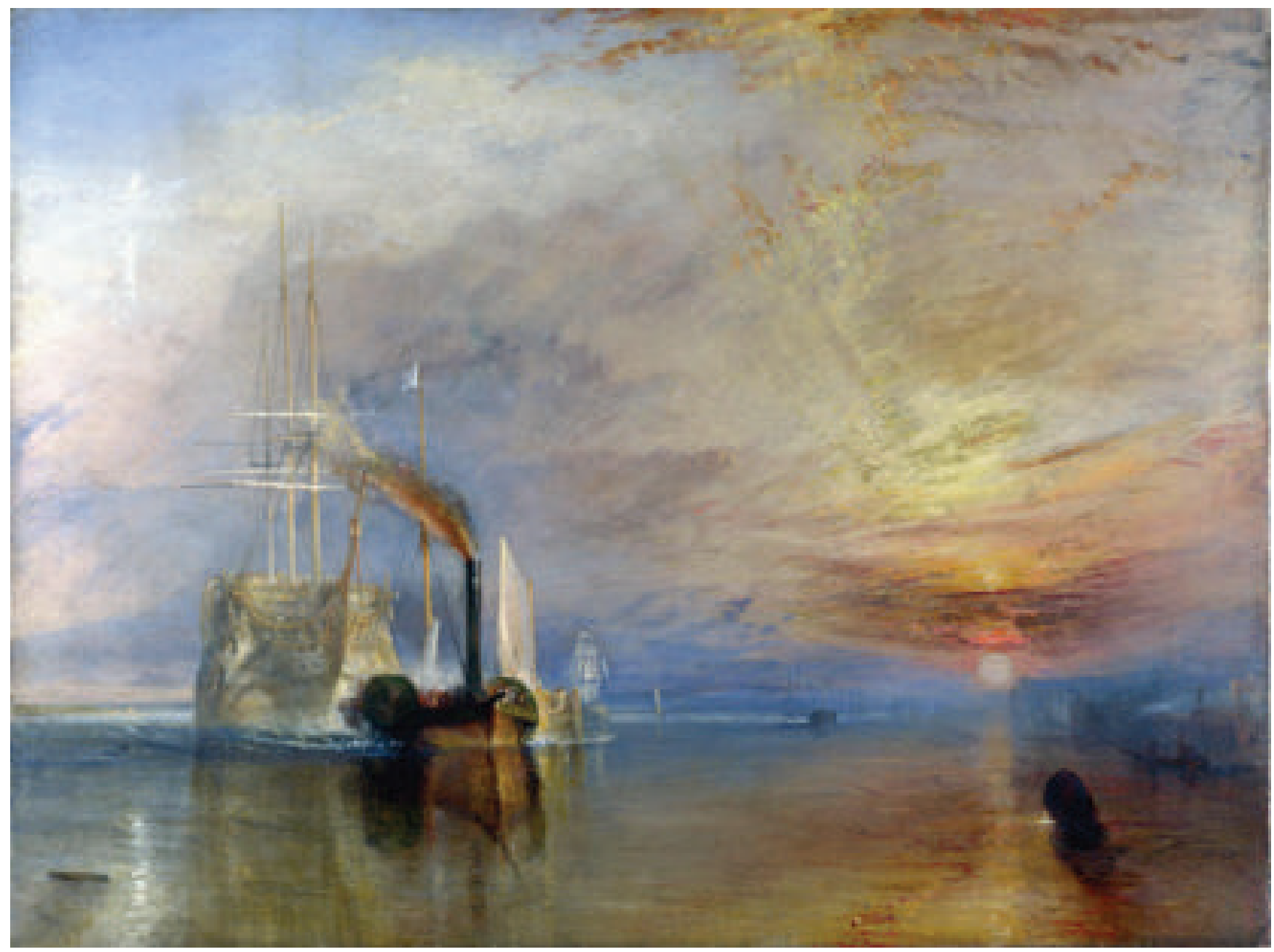

Figure 2 - The Fighting Temeraire tugged to her last berth to be broken up by J.M.W. Turner. 1839, $90.7 \mathrm{~cm} \times$ $121.6 \mathrm{~cm}$ (35.7 in × 47.9 in), National Gallery, London.

l'anniversaire de la Mère le 21 février 1967. Shraddhavan, le directeur du Savitri Bhavan où les peintures de Huta sont actuellement conservées à Auroville, dit que les « différences entre les [premières] versions [...] et les dernières sont frappantes. La Mère attribuait ce changement au "travail de la conscience" » 15.

Le 25 février 1972, la Mère acheva sa méditation sur la lecture du poème épique par une exposition qui incluait une présentation en diaporama des peintures accompagnées de musique et de la récitation du poème Savitri par la Mère. Ces peintures ne sont pas visibles pour les visiteurs (seules des copies imprimées sont exposées au Savitri Bhavan), ce qui laisse songeur quand on se rappelle que la Mère entendait ainsi «montrer au monde combien la conscience se développe ». Ce sujet demanderait d'autres investigations.

On m'a cependant donné la permission de partager quelques images d'une série ultérieure de peintures de Huta à partir des plus courts poèmes de Sri Aurobindo, où l'on peut voir entre autres le rendu des figures selon les caractéristiques que nous avons examinées : or ou rose dans les cheveux et la peau, la valeur des couleurs blanches et pastel, les figures "sans âge », parfois androgynes, la peau éclatante et les vêtements, etc ${ }^{16}$. "Le labeur d'un dieu » est un poème qui exprime très bien l'expérience spirituelle descendante de Sri Aurobindo pour diviniser la matière. La peinture de ce poème par

15 Courriel de Shraddhavan à l'auteur, 7 mai 2013.

16 Voir figures 3-5 


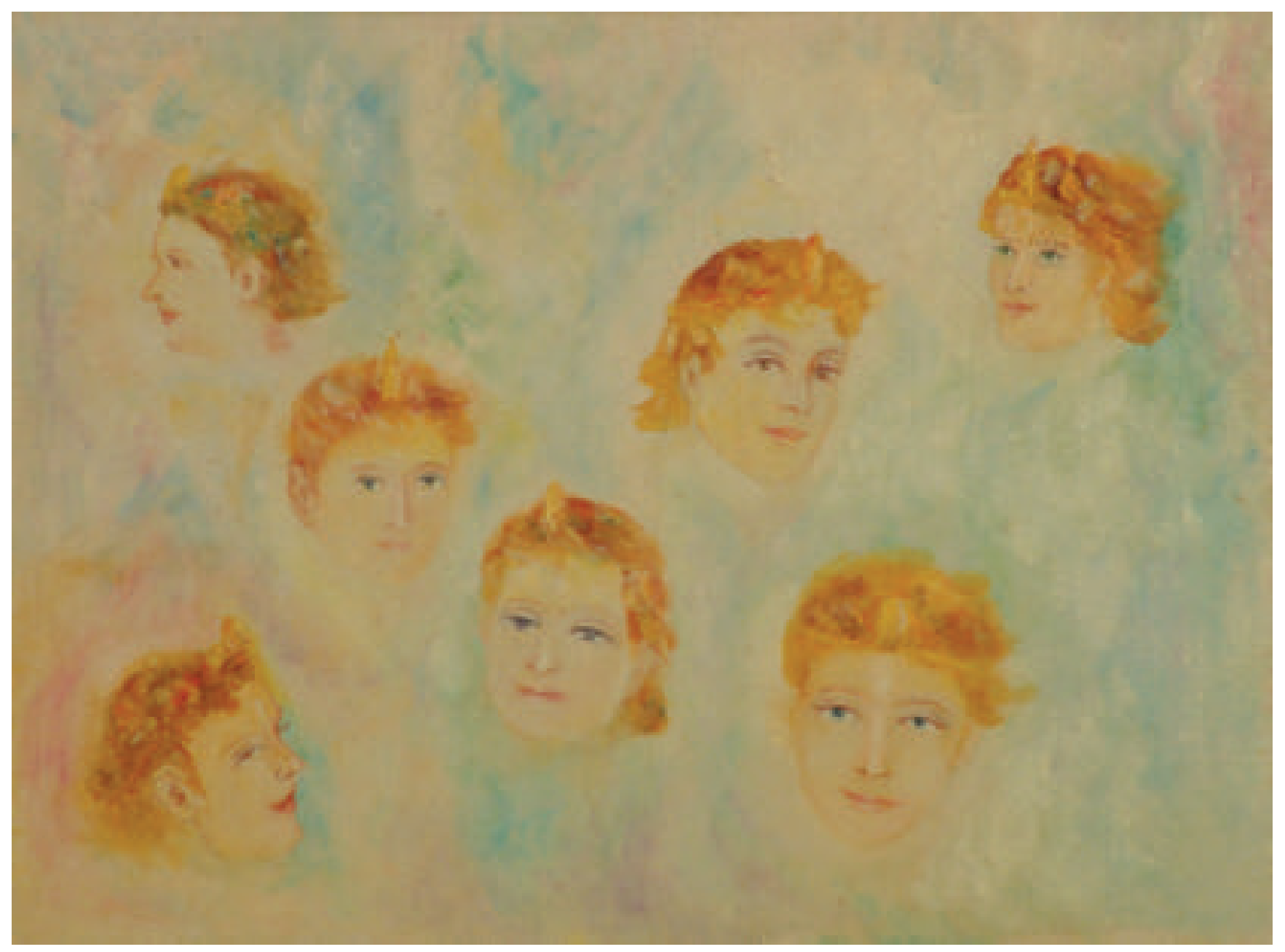

Figure 3 - "The Other Earths" by Huta. Oil on board. Photo by the Havyavāhana Trust, used with permission. "Calm faces of the gods on backgrounds vast/Bringing the marvel of the infinitudes."

Huta utilise les couleurs de l'or et du bleu (qui représentent les plans supramentaux et intuitionnels) dans une figure qui marche à l'intérieur de ce qui semble être une figure anthropomorphique dorée de la Terre, désirant Dieu avec la tête rejetée en extase (figure 4). La peinture de Huta semble répondre aux dernières Un peu plus et les portes de la vie nouvelle Seront taillées dans la lumière argent Avec son toit doré, ses sols de mosaïque En un vaste monde nu et resplendissant. strophes du poème de Sri Aurobindo:

Comme flammes aux royaumes de Vérité et Félicité :

Descendant un escalier or rouge, tournent Les radieux enfants du Paradis

Claironnant la fin des ténèbres.

Je laisserai mes rêves dans leur air argent,

Car sous une robe or et bleue

Tournera sur la terre belle et incarnée

Votre vivante vérité.

(Aurobindo, $1981: 125)^{17}$

17 Les deux derniers vers ont été retraduits de l'anglais. La Mère écrivit en commentaire : " L'air d'argent est le royaume spirituel. L'or est le supramental et le bleu est l'esprit. » (La Mère, 2009c : 357). 


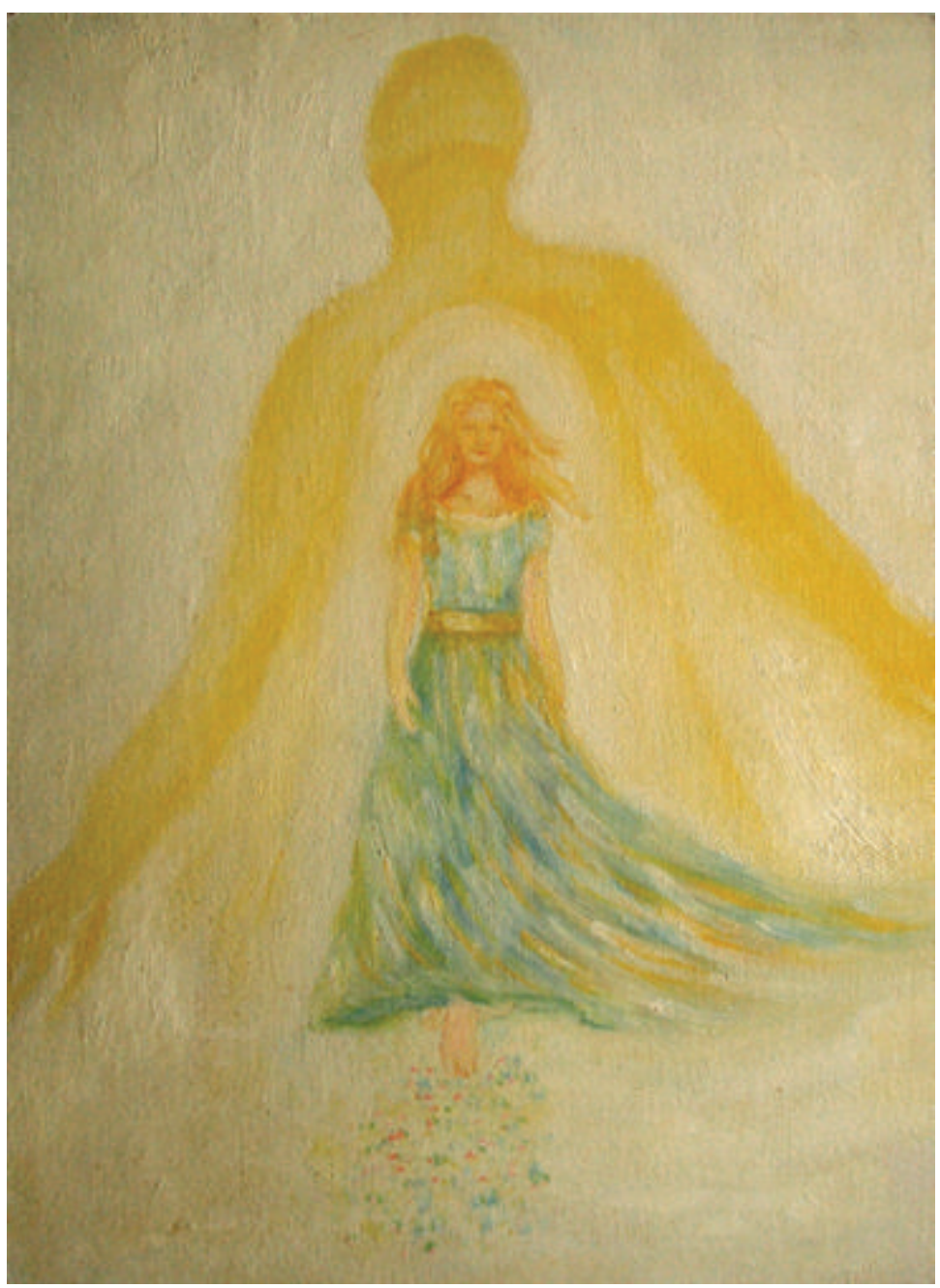

Figure 4 - "The Living Truth" by Huta. Oil on board. Photo by the Havyavāhana Trust, used with permission.

\section{Conclusion}

Le travail de la Mère avec l'art et d'autres artistes est une source déterminante de recherche pour comprendre sa pratique spirituelle et sa transmission. On pourrait dire que la façon dont le processus créatif d'une personne s'affine dans l'Ashram, reflète aussi son aspiration pour Dieu et réciproquement. Huta décrit cette expérience quand elle écrit : «non seulement la Mère m'enseigna à peindre, mais elle me donna aussi des leçons de vie : comment être modeste et persévérante dans mon effort pour atteindre la perfection et devenir une vraie artiste. » (Huta, 2001 : 11) Une « vraie artiste» de l'Ashram de Sri Aurobindo utilise son art comme une forme de yoga ; ce qui veut dire affronter et dépasser ses limitations propres, s'en remettre à la Mère et aspirer en sa conscience qu'un " Nouveau Monde » peut être créé aussi bien dans l'art que dans sa propre vie. 


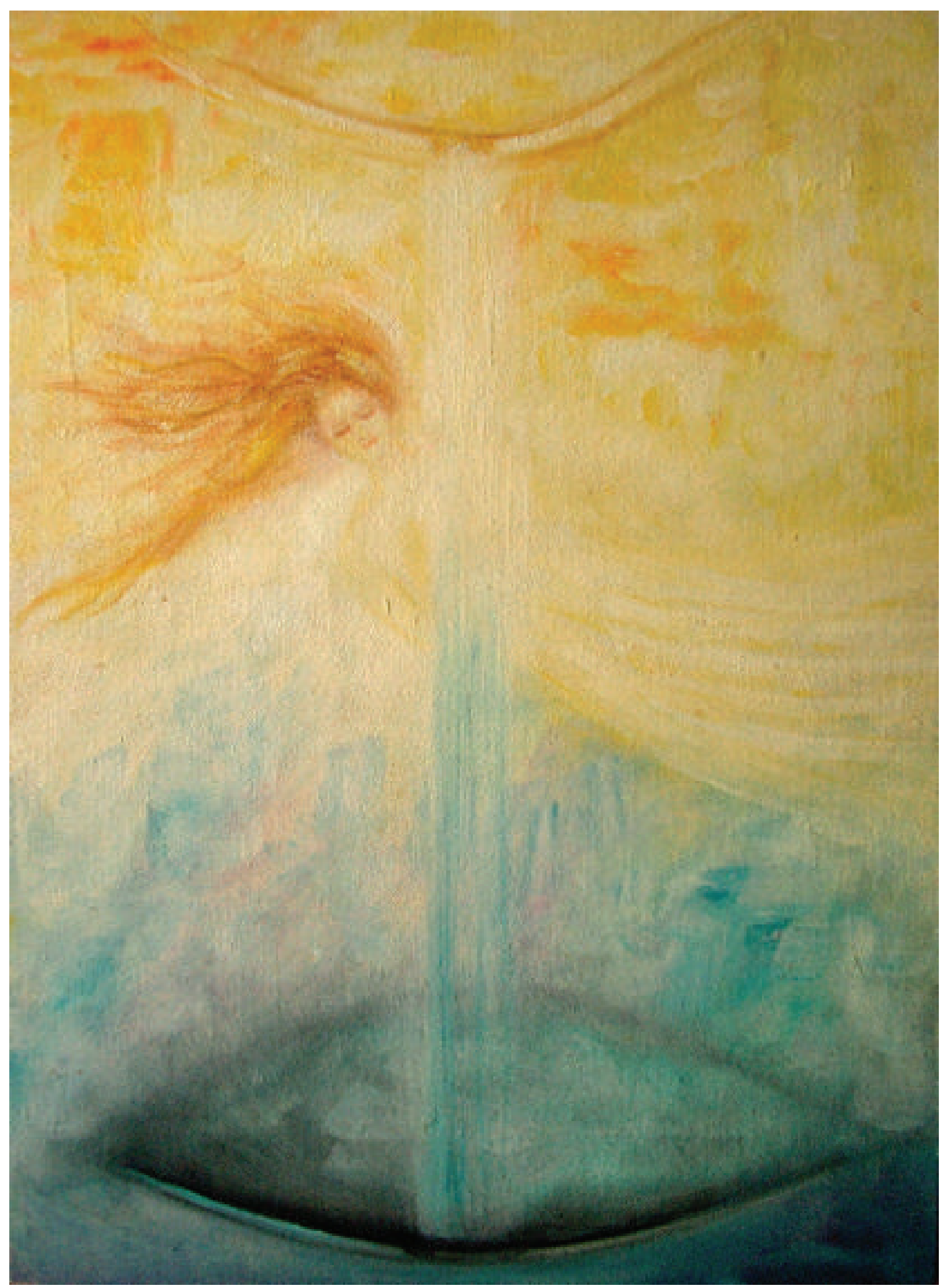

Figure 5 - "Musa Spiritus" by Huta. Oil on board. Photo by the Havyavāhana Trust, used with permission. "Into the gulfs of our nature leap,/Noice of the spaces, call of the Light!/Break the seals of Matter's sleep,/Break the trance of the unseen height." 
4 Présentation du

Centre Sri Aurobindo de Montréal et de

\section{son hybridité culturelle indo-québécoise}

David Brême

\section{Introduction générale au Centre Sri Aurobindo et à son contexte québécois}

Quand un mouvement spirituel mondialisé tel que celui de la Mère et Sri Aurobindo propose un enseignement universel, la tentation est grande d'en donner une définition générale. Toutefois, chaque personne et, a fortiori, chaque communauté de ce mouvement contribuent à le définir concrètement par la manière dont sont reçus les enseignements des fondateurs. Il s'agit là d'un processus d'hybridation'1 (Bhabha, 2007), où chacun intègre une culture reçue en fonction de celle dont il est issu. Plus précisément, Homi K. Bhabha conceptualise l'hybridité culturelle comme un processus qui crée un « tiers-espace » (2007 : 330) entre une culture apparemment dominante et une culture apparemment dominée :
« L'hybridité est plutôt pour moi le « tiersespace » qui rend possible l'émergence d'autres positions. Ce tiers-espace vient perturber les histoires qui le constituent, et établit de nouvelles structures d'autorité, de nouvelles initiatives politiques qui échappent au sens commun. " (Bhabha, 2006:97)

Dans le contexte de Montréal, les cultures originaires de l'Inde sont minoritaires par rapport aux cultures dominantes francophones québécoises et canadiennes-anglaises. Pour autant, l'Inde et sa culture peuvent susciter au Québec, comme dans d'autres pays occidentaux², la fascination ou, au contraire, l'aversion. Sabrina Zeghiche (2011) a souligné les représentations contrastées de la presse

\footnotetext{
${ }^{1}$ Homi K. Bhabha pense même que toute culture résulte d'un processus d'hybridation, ce qui revient à considérer l'identité culturelle comme factice, quelle que soit par ailleurs l'importance des déterminismes conduisant tout un chacun à s'identifier avec cette facticité.

2 Ainsi, selon les indianistes Michel Hulin et Christine Maillard (1996) soulignent la fascination qu'a exercée l'Inde sur la France et l'Allemagne dès le XIXe siècle dans L'Inde inspiratrice, réception de l'Inde en France et en Allemagne (XIXe-XXe siècles). L'indianiste Sen (2007), quant à lui, catégorise le discours anglais sur l'Inde en trois approches qualifiées de « conservatoriale », de «magistrale » et d'« exoticiste». Selon la sociologue Sabrina Zeghiche (2011), la première de ces approches consiste «à dresser un tableau de l'Inde à partir d'enquêtes, d'observations et de textes classiques. [...] La deuxième approche consiste à minimiser les apports culturels ou intellectuels de l'Inde et à en dresser un portrait peu flatteur. La troisième, au contraire, consiste à magnifier l'Inde et à exagérer ses qualités. »
} 
québécoise 3 dans un article intitulé « Lorsque l'“Occident" se tourne vers l'Inde : analyse de discours de la presse québécoise sur la gestion de la diversité culturelle et religieuse en Inde ». Dans le champ littéraire, Himabindu Timiri a analysé dans un mémoire de littérature les descriptions contrastées de l'Inde dans trois romans québécois, soit pour glorifier sa culture, soit pour généraliser hâtivement des comportements indiens, tout en édifiant une représentation alliant «pauvretéspiritualité » (2008: 119).

Sur le plan socioreligieux, on retrouve ces représentations collectives contradictoires de l'Inde au Québec dans le contexte d'un ashram montréalais étudié par Karine Geoffrion (2011), qui s'est intéressée au rôle du gourou montréalais Sri Adi Dadi auprès de ses 250 disciples. Elle souligne que "le concept de gourou, relevant principalement des philosophies orientales, peut avoir une connotation péjorative en Occident. [...] Pourtant, à l'ashram, le gourou est non seulement accepté en tant que tel, mais il fait l'objet de la dévotion de ses fidèles dont une majorité le suit depuis plus de 20 ans. [...] [Ainsi, dans] le contexte québécois, la plupart de [ses] informateurs agissent de façon contradictoire dans leur rapport à la religion. Élevés dans la religion catholique, beaucoup s'en sont détournés par rejet de l'autorité ecclésiastique et des doctrines ; [c'est pourquoi] plusieurs disciples de l'ashram semblent vivre des contradictions dues au contexte spirituel et religieux du Québec» (Geoffrion, $2011: 33-34)$.

On ne peut certes généraliser ces cas d'étude particuliers à la réception montréalaise de l'Inde et de sa culture, mais ils sont autant d'indices qui éveillent notre vigilance sur une attitude qui serait soit laudative, soit condescendante vis-à-vis de la religion et de la culture de l'Inde.

Un autre préjugé serait de penser qu'il existerait une culture essentiellement liée à un territoire géographique déterminé ; cela était peut-être approximativement vrai avant l'interaction mondiale des échanges et l'est probablement toujours pour certains terrains, mais rarement dans le contexte de métropoles importantes en interdépendance historique avec de multiples cultures. L'un des intérêts ethnographiques du Centre Sri Aurobindo fondé en 1965 avec l'accord de la Mère est son ancienneté relative en tant que centre spirituel montréalais en relation à l'Inde, sa relation soutenue avec l'Ashram de Sri Aurobindo à Pondichéry et sa position au Québec, au Canada et en Amérique du Nord : cela en fait un site remarquable croisant plusieurs cultures, à prédominance québécoise, en continuité avec le contexte montréalais majoritairement francophone à l'est de la rue Saint-Laurent.

Lassociation Auroville Canada, issue du Centre Sri Aurobindo à Montréal, a été déclarée de manière indépendante le 10 avril 1971 et compte actuellement une trentaine de membres actifs, dont quelques-uns participent aux deux organismes. L'activité 4 et l'histoire de cette association ne seront pas abordées ici, mais elles témoignent du besoin d'avoir au Québec, après cette date, au moins deux organismes ${ }^{5}$ représentant de différentes manières le Mouvement spirituel Mère-Sri Aurobindo. On pourrait le rapprocher de l'émergence en Inde de deux espaces distincts de ce mouvement, soit Auroville et l'Ashram de Sri Aurobindo à Pondichéry.

\footnotetext{
3 Sabrina Zeghiche a effectué cette enquête avec un échantillon de 54 articles de presse québécoise sur l'Inde entre 2000 et 2010.

4 Cette association finance des projets auroviliens et propose à ses membres des sorties et des lectures de Sri Aurobindo.

5 Il y eut aussi dans les années 70 et 80 le restaurant « Op-Ti-Zoizo », qui se reliait à ce mouvement. Il n’existe plus.
} 
Par ailleurs, le Centre Sri Aurobindo est un exemple spécifique du renouveau de l'horizon religieux et spirituel de la société québécoise dans le contexte de la Révolution tranquille des années 1960 et 1970, tandis qu'un processus de sécularisation nationalisait à l'échelle provinciale québécoise l'éducation et maints services sociaux liés jusqu'alors à l'Église catholique. Celle-ci se vit rejetée par une partie de la population à ce moment. Ainsi, une répondante québécoise, Isa, me disait en 2013 : «On est au Québec, on est de la génération qui a abandonné une religion. »

Enfin, la Mère a très tôt rendu accessibles les écrits de Sri Aurobindo au public francophone par ses traductions françaises et par la littérature de son disciple français Satprem (Bernard Enginger, 1923-2007). Cet accès linguistique francophone fut déterminant pour la plupart des Québécois de cette époque, pour lesquels la langue française constitue un marqueur identitaire déterminant. La majeure partie des répondants interviewés ont mentionné la lecture de Sri Aurobindo ou l'aventure de la conscience, écrit par Satprem en français, comme un élément déclencheur décisif de leur quête spirituelle. Laccent porté sur la lecture régulière et l'étude des œuvres des fondateurs est d'ailleurs une des caractéristiques des données recueillies sur ce site de 2013 à 2017.

J'ai effectué dix entrevues en juin 2013 et j'ai eu accès aux archives du Centre Sri Aurobindo dans le cadre de ma recherche doctorale en automne 2016. J'étais alors dans une posture d'observation participante. Puis j'ai recueilli un ensemble d'informations complémentaires auprès des membres du Centre depuis septembre 2015, moment à partir duquel j'ai adopté une posture plus engagée vis-à-vis de mon terrain.

Si on se remet dans le contexte des années 60, l'affinité linguistique francophone a été certainement décisive dans le choix de Québécois d'aller en Inde à Pondichéry plutôt qu'ailleurs. Jean-Jacques Chapdelaine, sculpteur sur glace et grand athlète, aurait été le premier Québécois à visiter l'Ashram de Sri Aurobindo à Pondichéry en prenant le bateau à la fin des années cinquante. Guy Lafond, traducteur de Savitri et secrétaire à la fondation du centre, aurait été le second.

Sur le plan local, il faut bien avoir à l'esprit que, pendant les années soixante, la ville de Montréal était québécoise francophone à l'est de la rue Saint-Laurent et anglophone à l'ouest de cette même rue, avec tout juste suffisamment de Juifs (parlant le yiddish), de Portugais, d'Italiens et d'autochtones au milieu de ces deux solitudes linguistiques prédominantes pour leur rappeler qu'elles procédaient de deux complexes coloniaux britannique et français en rivalité. Il s'ensuivit dans les années soixante quelques voies de fait (manuels scolaires en anglais brûlés, etc.) et échauffourées sur la rue SaintLaurent notamment. C'est dans ce contexte sociopolitique mouvementé et hybride que s'est construit le Centre Sri Aurobindo à l'initiative de Madeleine Gosselin (1927-2014). Entre-temps, Montréal est devenu plus polyglotte, mais le Centre Sri Aurobindo se situe à l'est de la rue Saint-Laurent, dans un quartier très majoritairement francophone. 


\subsection{Le Centre Sri Aurobindo et sa construction communautaire québécoise (1965-1979)}

Le Centre Sri Aurobindo a été fondé par Madeleine Gosselin suite à un voyage en 1965 en Inde, où elle rencontra la Mère. Une brève chronologie permet de donner quelques points de repère sur l'histoire de ce centre (voir page suivante).

A u jourd'h u i encore $^{6}$, dans le hall d'entrée servant de vestiaire aux personnes accueillies le mercredi et le dimanche soir pour des séances de méditation collective, figurent sur un tableau les bénédictions de la Mère à l'adresse de Madeleine Gosselin et à l'occasion de son anniversaire le 3 avril 1965 , jour de la

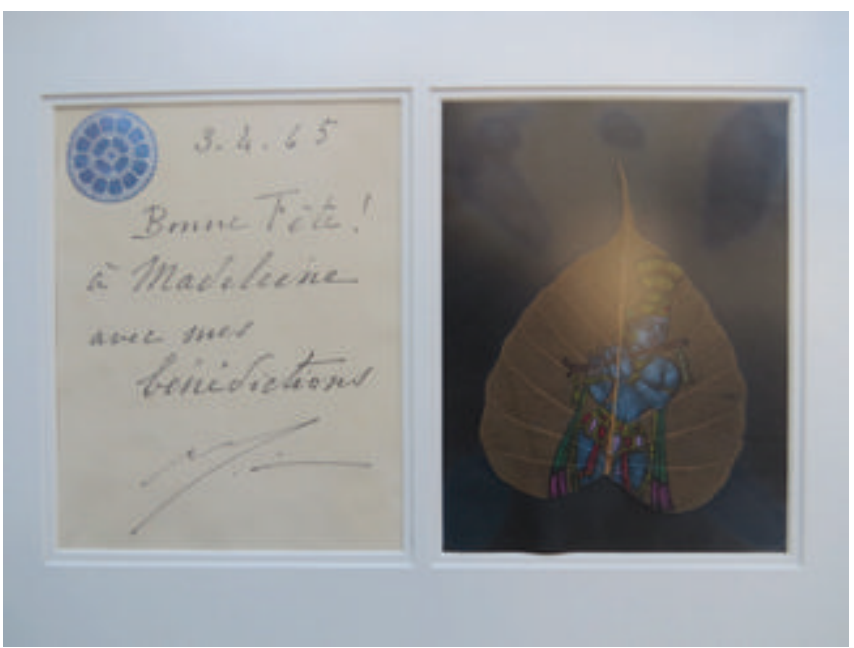

Les bénédictions de la Mère à Madeleine Gosselin. fondation du centre.

La Mère lui avait d'abord donné oralement son accord pour ouvrir le Centre Sri Aurobindo à Montréal lors de son premier voyage (Satprem, 1981a :127), en préconisant, d'après une répondante 7 , que Guy Lafond en soit le secrétaire. Guy Lafond était présent à l'Ashram de Pondichéry avant Madeleine Gosselin et détenait une bonne connaissance de la philosophie de Sri Aurobindo. Devenu secrétaire du Centre montréalais, il s'est notamment fait connaître ultérieurement par sa traduction de Savitri (Sri Aurobindo, 1951/2005) aux éditions
Christian Feuillette 8 . On peut d'ailleurs considérer que cette œuvre est « le texte sacré » du mouvement spirituel Mère-Sri Aurobindo.

Madeleine Gosselin reçut également, en 1965, une lettre de Pavitra, secrétaire de l'Ashram de Sri Aurobindo à Pondichéry, lui donnant cette fois l'autorisation écrite d'ouvrir le Centre Sri Aurobindo. Sa relation $a v$ e $\mathrm{l}$ a M ère se manifestait notamment par un échange épistolaire régulier et au moins trois voyages à Pondichéry, en 1967, 1970 et 1971 (Gosselin, s.d). Elle rédigea par ailleurs une lettre dans laquelle elle décrit les débuts du Centre qui donne des indications utiles pour comprendre sa relation avec la Mère et la dynamique initiale du Centre :

«Ce que je voudrais que tu comprennes, c'est que je ne suis pas l'instigatrice du Centre. Il était là et m'attendait pour se manifester dans la matière, rien de plus. Je n'en ai été que l'instrument et je crains de n'être que cela comme tant d'autres, comme tous ces jeunes qui venaient travailler bénévolement à la rénovation, qui travaillaient jusqu'à épuisement et tous

6 Le 9 novembre 2017.

7 Entrevue de terrain 2013.

8 Christian Feuillette est un Français implanté au Québec depuis plus de quarante ans qui a été un membre actif de la première heure au Centre Sri Aurobindo à Montréal, remplaçant le secrétaire Guy Lafond à partir de 1970, a notamment contribué à l'institutionnalisation légale du Centre (cf. paragraphe 4.2) et à son institutionnalisation symbolique en faisant la demande de reliques à l'Ashram de Sri Aurobindo à Pondichéry à titre de vice-président du Centre. Il a ensuite quitté le Centre et s'est investi dans le projet d'Auroville. 


\section{Chronologie Centre Sri Aurobindo de Montréal $\downarrow$}

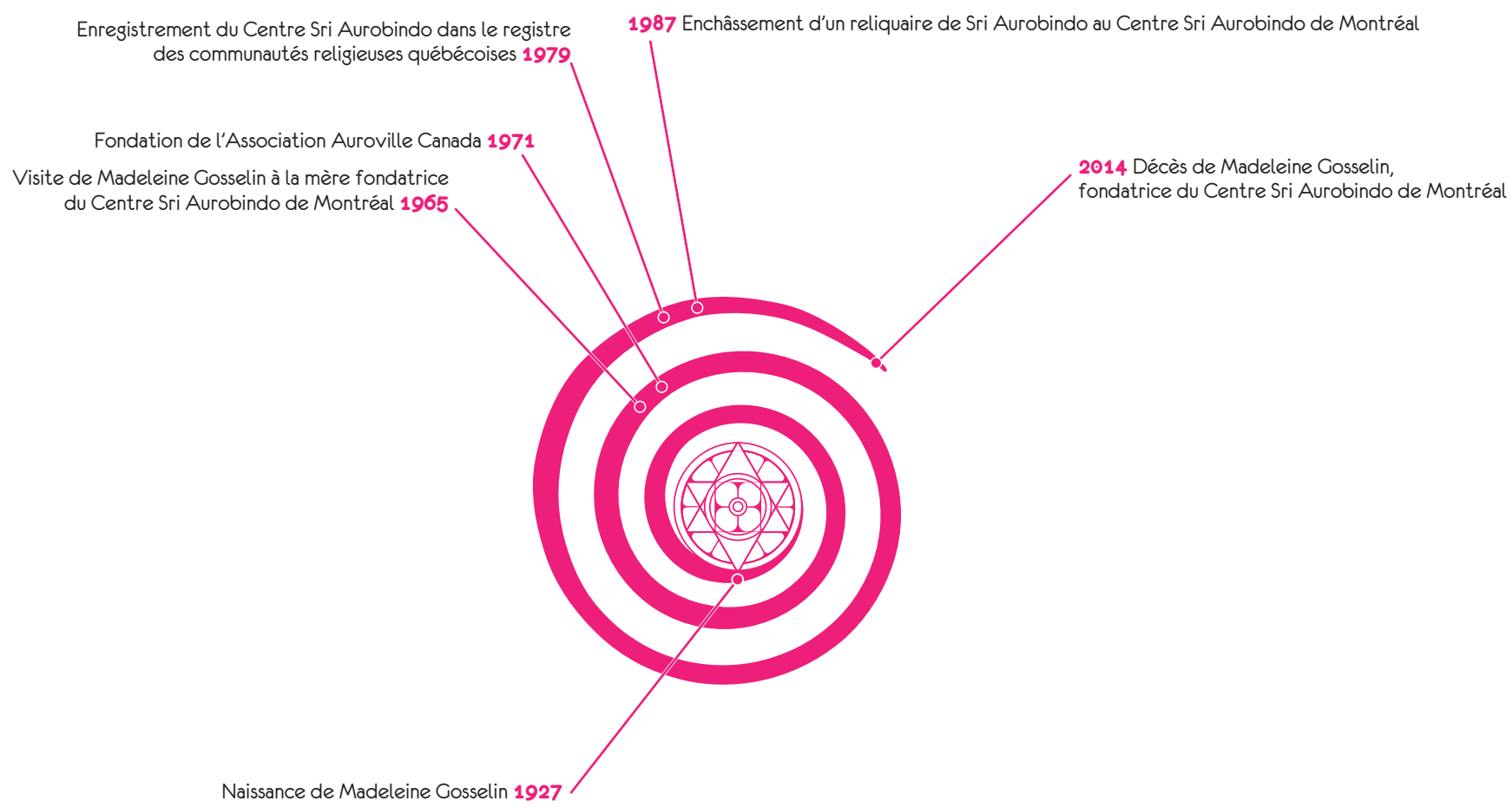

ceux aussi qui collaboraient par des dons que le Centre n’a jamais sollicités ${ }^{9}$.»

La rénovation à laquelle elle fait allusion est l'ensemble des chantiers de construction et de réhabilitation de maisons acquises à partir de 1974 pour fonder la communauté du Centre de Montréal. Le discours épistolaire de Madeleine Gosselin parle ultérieurement de l'« Amour» de la Mère reçu par tous les bénévoles pendant ces chantiers et de sa fonction instrumentale, ainsi que celle des membres du Centre Sri Aurobindo et de ses sympathisants.

Comment ces sympathisants ont-ils vécu leur relation à la Mère de leur côté? Béatrice, une membre du Centre déjà active dans les années soixante-dix, m'a informé davantage à ce sujet. Elle nomme Madeleine Gosselin par son diminutif affectueux «Mado » et souligne que la difficulté de cette époque tenait surtout à la dynamique collective présente :

" Dans ce groupe d'une vingtaine de personnes, il y avait toujours quelqu'un qui touchait ton ego. C'était très difficile pour tout le monde. L'action de la lumière consiste à éclairer ce qui doit être transformé en toi, que tu dois offrir à la Mère qui te transforme. Lorsque je suis arrivée, Mado donnait trois cours de hathayoga chaque soir, cinq soirs par semaine, un cours le dimanche matin et un avant la méditation du soir. D’autres personnes se sont jointes, telles que Guy Lafond qui a donné des cours au Centre pendant quelques années. Après les cours, nous aimions rester dans cette atmosphère et nous nous attardions. Mado était sensible à ce besoin. Un jour il y a eu une maison à vendre derrière le Centre. Mado a finalement décidé de l'acheter : cela permettrait à une collectivité déjà existante de se concrétiser. C'était la réponse à un besoin de demeurer dans cette atmosphère de Mère et de Sri Aurobindo. C'était en 1974. Mado, toujours disponible, répondait de son mieux à tous ceux qui venaient, par

9 Les lettres et textes de Madeleine Gosselin cités dans ce chapitre sont conservés dans les archives du Centre Sri Aurobindo et ont été consultés le 8 novembre 2016. 
sa présence, son écoute, son travail et ses cours de hatha-yoga. »

Aux débuts du Centre de Montréal, Mado donna ainsi plus de quinze cours de hatha-yoga par semaine et acheta les biens de la communauté en son nom propre.

De 1974 à 1979, une autre forme d'activité que le yoga vit le jour, avec la rénovation de maisons développant la communauté, comme le relate Béatrice :

« Les premières personnes ont emménagé [dans la maison nouvellement acquise] vers juin 1974. Au cours des mois et des années suivantes, plusieurs autres maisons ont été achetées à très bas prix étant souvent dans un état déplorable : une, entre autres, dont les locataires étaient partis en laissant les robinets ouverts en plein hiver, ce qui en avait fait un château de glace. Nous étions jeunes, pleins d'énergie et avec une même "aspiration" au fond du cœur, nous nous sommes attelés à la tâche : rénovations des maisons, cuisine et entretien des vêtements et des lieux. Il y avait beaucoup à faire. »

Deux photographies copiées des archives $\mathrm{du}$ Centre reflètent en partie l'atmosphère présente dans cette communauté à cette époque

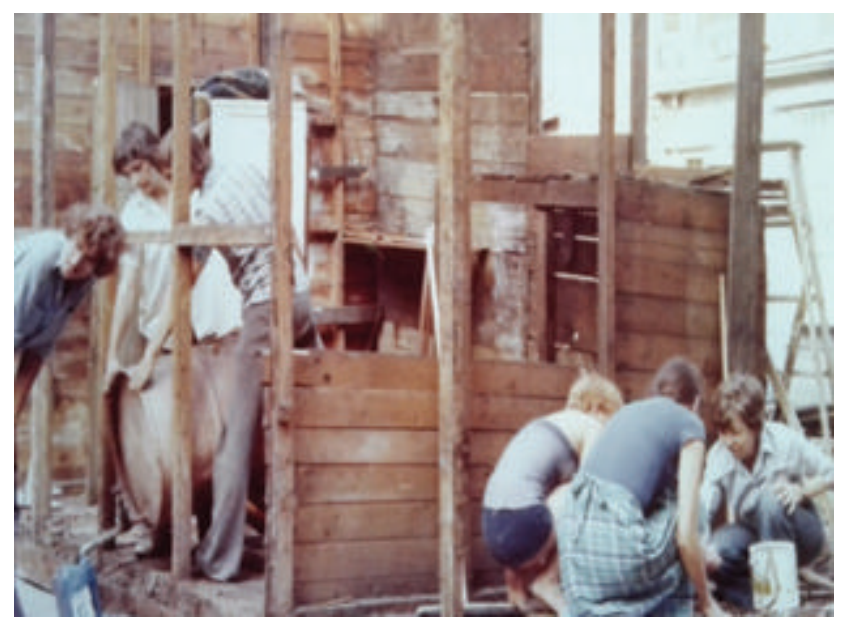

Des chantiers du Centre Sri Aurobindo entre 1974 et 1979.

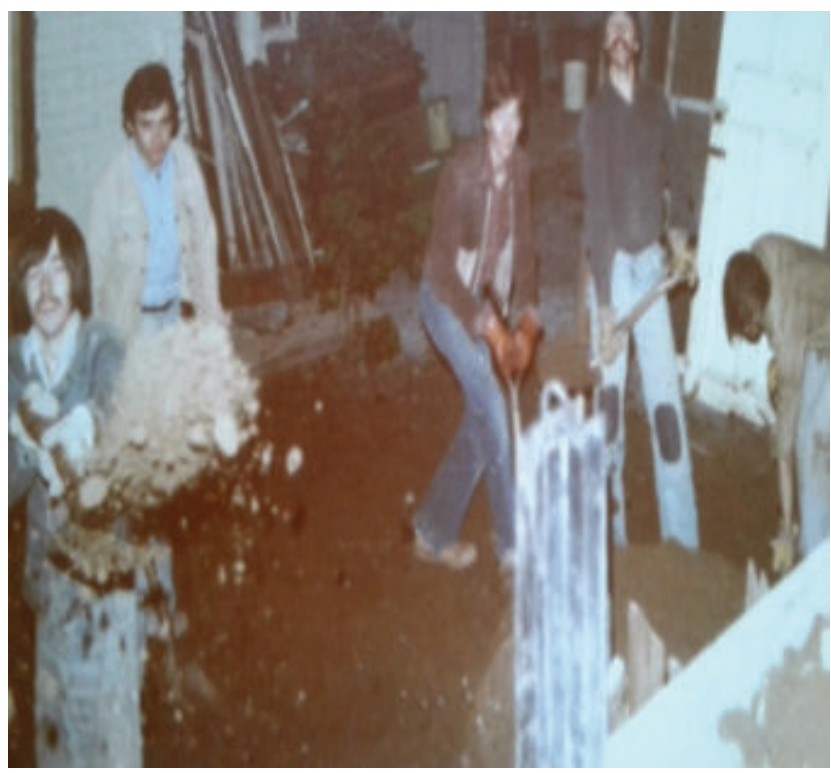

des seventies, près du coin formé par les rues Saint-Denis et Rachel.

Le chantier de rénovation sur la photo de gauche est celui de l'ancienne maison qui avait l'air d' " un château de glace ». " La plupart des gens travaillaient beaucoup plus que quatre heures par jour » selon Béatrice et la plupart des bénévoles exerçaient une activité professionnelle en dehors du Centre Sri Aurobindo, assurant ainsi leur autonomie et celle du Centre. Il s'agit là d'une caractéristique qui distingue le Centre de l'Ashram de Sri Aurobindo et d'Auroville, où les principales activités des membres se déroulent surtout in situ.

Ces aspects de la vie du Centre de Montréal apparaissent comme des conséquences indirectes de la Révolution tranquille québécoise, durant laquelle les structures traditionnelles de cette société ont été rejetées, ce qui a suscité, pour le groupe constituant le Centre Sri Aurobindo de Montréal, le besoin de recréer un horizon collectif spirituel à partir de l'Inde, ou en l'occurrence de l'enseignement de la Mère et de Sri Aurobindo. Cette époque était aussi propice à la création de communautés, susceptibles en retour de motiver chaque individu dans sa quête. À la lecture de cette dernière interprétation, la répondante Béatrice a précisé : 
« Je pense plutôt que c'est cette Nouvelle Conscience qui se répand sur la terre qui a provoqué la Révolution tranquille et favorisé l'émergence d'une « aspiration » déjà présente, ce qui a amené les gens au yoga de Sri Aurobindo. La vie en commun n'était pas l'objectif, mais la conséquence de notre besoin de vivre dans l'atmosphère de la Mère. "

Quelle que soit la façon dont on interprète la Révolution tranquille et ce qui a déterminé la fondation du Centre Sri Aurobindo de Montréal avec sa communauté, le lien entre ces événements paraît fondamental. La répondante Isa le signifiait à sa manière en disant : « On est de la génération qui a abandonné une religion et qui est passée tout de suite à une spiritualité. » Le régime de Duplessis, Premier ministre québécois de 1944 à 1959, précédant Jean Lesage, était en effet allié à l'Église catholique, qui contrôlait depuis la fin du XIX ${ }^{\mathrm{e}}$ siècle l'éducation au Québec et exerçait un pouvoir social patriarcal important sur cette société. La vague féministe des années soixante allait en partie renverser cette tendance. Elle allait notamment permettre :

- En 1964, la reconnaissance de la capacité juridique des femmes mariées au niveau de la province du Québec,

- En 1968, l'établissement d'un droit au divorce simplifié au niveau fédéral,

- Un accès aux études supérieures, graduellement plus important.

- Une capacité accrue des femmes à prendre leur place au sein de la société québécoise et canadienne.

Isa fait ainsi part de sa motivation féministe dans sa quête spirituelle et son choix de suivre l'enseignement de Sri Aurobindo :

«La question de la supposée infériorité des femmes, mon refus de croire que j'étais un être inférieur et ma révolte contre tout ce qui aurait voulu m'en faire devenir un a certainement joué un rôle dans mon rejet $\mathrm{du}$ catholicisme, des dieux sexistes et de bien des sornettes philosophiques, psychiatriques et psychologiques. Certaines philosophies ou spiritualités orientales me sont apparues plus neutres, un peu moins machistes, mais ce n'est qu'à la lecture de Sri Aurobindo, vers l'âge de 21 ans, que j'ai pu espérer d'un homme dit "sage" la confirmation de ce que je ressentais moi-même, en tant qu'incarnée dans un corps de femme. »

Le Centre Sri Aurobindo à Montréal, en ayant édité en son nom comme seul ouvrage un recueil de textes de Sri Aurobindo et de la Mère intitulé La femme et en ayant toujours eu, jusqu'à ce jour au moins, une femme en position d'autorité responsable de son administration générale, s'inscrit partiellement dans ce courant féministe québécois dit parfois de « la seconde vague » des seventies, par lequel les femmes ont pris davantage leur place socioprofessionnelle.

Lattrait pour les spiritualités orientales s'exprimait au Québec au moins depuis la première ouverture d'un ashram de Sivananda (Vishnudevananda, 2017) à Val Morin en 1962, spécialement parce que ces spiritualités investissent le corps par le yoga, comme c'était le cas pour le Centre Sri Aurobindo où Madeleine Gosselin enseignait le yoga.

Madeleine Gosselin écrivit en septembre 1973 à André Morisset ${ }^{10}$, secrétaire auprès de la Mère, afin que le Centre soit officiellement et institutionnellement rattaché à l'Ashram de Pondichéry:

« Le Centre Sri Aurobindo de Montréal voudrait légaliser devant notaire son statut social. [...] [À] cause d'activités accrues (tel le comptoir d'artisanat), il est devenu impérieux que nous obtenions une Charte auprès de notre gouvernement. Comme vous pouvez le constater par la copie de la

10 André Morisset était aussi le fils unique de Mirra Alfassa, la Mère. 
lettre du notaire préposé à ces démarches, que nous joignons à la présente, il faut d'abord enregistrer auprès de notre gouvernement la Charte dite "de la maison-mère ». Or, comme le Centre de Montréal fut fondé sous l'approbation directe de Mère, et qu'un de ses buts primordiaux est de servir de lien entre ses membres et l'Ashram de Pondichéry, nous vous demandons, si c'est possible, de nous faire parvenir la Charte même de l'Ashram afin que nous puissions la déposer au bureau d'enregistrement de notre gouvernement. Nous [...] serions considérés comme affiliés directement à l'Ashram. Pourriez-vous, en plus, nous envoyer une lettre spécifiant que Mère a accepté que $\mathrm{M}^{\text {me }}$ Gosselin ouvre un Centre Sri Aurobindo de Montréal ? Le seul mot que nous ayons à cet effet est une lettre de Pavitra datant de 1965. »

Sa volonté de légaliser les statuts du Centre indiquait aussi son sens des responsabilités collectives : elle entendait que soit posé un cadre légal délimitant les responsabilités du Centre Sri Aurobindo et de ce fait les siennes, en tant que sa présidente. Madeleine Gosselin avait aussi désiré inscrire le Centre de Montréal dans le cadre institutionnel et légal du Québec en demandant la reconnaissance de l'Ashram de Sri Aurobindo à Pondichéry en tant que «maison-mère».

Le 22 septembre 1973, André Morisset, en tant que secrétaire de la Mère - et de sa mère répondit:

"Si Mère vous a donné la permission d'ouvrir un centre à Montréal, cela ne veut pas dire que ce centre peut être considéré comme le prolongement de l'Ashram. L'Ashram n'a pas de succursales [...]. Cela n'empêche pas que votre centre puisse avoir une existence légale11. »

Cette lettre écrite deux mois avant le décès de la Mère clarifiait le statut du Centre Sri Aurobindo vis-à-vis de l'Ashram. Auparavant, l'échange épistolaire régulier que Madeleine Gosselin avait avec la Mère lui permettait d'envisager le Centre Sri Aurobindo comme un prolongement de son Ashram, mais ce qui pouvait être vrai du fait de la similitude des objectifs partagés ne l'était pas nécessairement au niveau légal, où chaque nation (et en l'occurrence au Canada, chaque Province) a son dispositif législatif qui lui est propre.

\subsection{Institutionnalisation du Centre Sri Aurobindo et sa vocation (1979-2017)}

Les démarches de Madeleine Gosselin afin de faire reconnaître légalement le Centre de Montréal aboutirent le 6 mars 1979 à des lettres de patente provenant du ministère québécois des Consommateurs, des Coopératives et des Institutions financières. Ces lettres de patente reconnaissent le Centre en tant que corporation religieuse avec l'objet suivant :

« Organiser, administrer et maintenir la congrégation et l'œuvre de Sri Aurobindo dont les fins sont la charité, l'enseignement, l'éducation, la religion et le bien-être. » (Ministère québécois des Consommateurs, des Coopératives et des Institutions financières. 1979)

Quoique le terme "religion " soit généralement rejeté par les membres du Centre pour ses connotations dogmatiques et historiques, ce cadre légal fut adopté par ces derniers, puisqu'il était le plus approprié à la situation du Centre.

Madeleine Gosselin voulut surtout légaliser les activités de "comptoir d'artisanat»,

11 Cette lettre se trouve dans les archives du Centre Sri Aurobindo de Montréal et a été consultée le 8 novembre 2016. 
qui participaient de l'économie informelle du groupe, et asseoir dans la durée la légitimité charismatique du Centre en l'adossant au droit québécois, quitte à concéder la dénomination officielle de " corporation religieuse». De plus, la communauté du Centre Sri Aurobindo se déployait après la première maison acquise en 1974. Cette expansion immobilière, faite initialement au seul nom privé de Madeleine Gosselin, requérait d'être transférée par un statut corporatif légal adéquat, afin que le Centre Sri Aurobindo de Montréal puisse officiellement prendre sa vocation de congrégation de Sri Aurobindo et ne pas être confondu avec la propriété de Madame Gosselin.

La présence de Guy Lafond, en tant que secrétaire du Centre Sri Aurobindo et professeur de yoga, était précieuse à ce titre : Madeleine Gosselin n'était pas la seule autorité morale représentant Sri Aurobindo et la Mère au Québec. Guy Lafond avait par exemple écrit le programme du Centre Sri Aurobindo sous cette forme :

" Le Centre Sri Aurobindo a été fondé, sous l'égide de la Mère, afin:

- De créer un lieu de silence où chacun, hors de toute sollicitation, peut se retrouver face à son aspiration la plus profonde.

- De créer un lien physique avec l'Ashram de Pondichéry.

- De diffuser la pensée de Sri Aurobindo, de Mère, du yoga intégral selon la demande.

Ce programme constitue notre Matrimandir particulier. " (Lafond, s.d)

Le terme "Matrimandir » renvoie à la géode de la communauté d'Auroville, qui a demandé 40 ans de construction collective et qui, en tant que centre symbolique, a constitué un enjeu identitaire et un objectif important pour cette communauté. Soulignons les énoncés «créer un lieu de silence » et «diffuser la pensée de Sri Aurobindo, de Mère » qui constituent le programme du Centre à Montréal. Ce programme fait écho à une présentation inaugurale du Centre par Madeleine Gosselin :

« Comme le premier pas à franchir dans l'expérience que nous offre Sri Aurobindo est d'entrer en contact avec son monde intérieur individuel, chacun à sa façon; et comme cette entrée en soi s'effectue par et dans le Silence, l'activité centrale du Centre Sri Aurobindo, et, pour débuter, la seule, sera une période de silence hebdomadaire. Tous ceux qui le veulent peuvent $y$ participer. »

Les méditations collectives régulières silencieuses proposées gratuitement de façon hebdomadaire accomplissent la part $\mathrm{du}$ programme consistant à "créer un lieu de silence ». Quant à la dynamique de travail collectif au service de la diffusion des œuvres de la Mère et de Sri Aurobindo, elle est par exemple manifeste par l'ouverture journalière de la librairie ${ }^{12}$ depuis 1981 et le bénévolat en cuisine collective soutenant alimentairement les volontaires le jour de leur service.

La volonté « de créer un lieu de silence [...] hors de toute sollicitation " au sein d'une vie communautaire peut s'entendre comme le besoin de remplacer, dans le contexte québécois, le vide créé par le rejet de la religion catholique. Socialement, nous pouvons le concevoir de ce fait comme un " espace tiers » hybride au sens donné par Homi K. Bhabha, syncrétisant la culture québécoise issue de la Révolution tranquille et la culture yogique du silence, du moins celle issue du yoga de Sri Aurobindo et de la Mère. La volonté d'établir une forme de silence méditatif invite chacun à se faire face, en respectant ainsi à la fois le double rejet des

124125 rue Saint-Denis, H2W 2M7, Montréal, QC. La librairie, dite «la boutique », ouverte depuis 1981, vend aussi de l'artisanat, qui provient d'Auroville et de l'Inde en général. 
discours des religions provenant du contexte social de la Révolution tranquille au Québec et de la Mère ${ }^{13}$ en Inde. De plus, cette volonté correspond à ce que préconisait la Mère, par exemple dans son message du 11 février 1972 : "Seigneur Suprême, apprends-nous à être silencieux, afin que dans le silence nous puissions recevoir ta force et ta volonté. » (Satprem, 1982 : 57).

La construction de ce lieu de silence s'est notamment opérée par un rite d'ancrage ${ }^{14}$ (Bradley, 2015), c'est-à-dire un rite par lequel une communauté symbolise sa volonté de s'établir de façon pérenne en un lieu donné. Linscription juridique québécoise du Centre en 1979 et l'achat du bâtiment dans lequel est la salle de méditation, auparavant loué, ont contribué à cet ancrage, mais ne constituent pas pour autant un rituel symbolisant l'ancrage du sacré, c'est-à-dire d'une reconnaissance du divin dans un espace défini par sa matérialisation symbolique. Louverture d'une salle de méditation définissait d'emblée un tel espace et les cours de yoga de Madeleine Gosselin reliés à la Mère matérialisaient peut-être initialement cette dimension sacrée. Elle demeurait toutefois éminemment subjective, propre à la réceptivité de chacun des membres. En 1973, peu avant le décès de la Mère, Madeleine rechercha un rattachement plus objectif - juridique - à l'Ashram de Sri Aurobindo à Pondichéry. De son côté, Guy Lafond symbolisait le projet du Centre Sri Aurobindo de Montréal par la métaphore du Matrimandir d'Auroville. Ces discours voulant rattacher le Centre Sri Aurobindo de Montréal soit à Auroville soit à l'Ashram de Sri Aurobindo à Pondichéry montrent que son identité propre n'était pas encore bien ancrée.

Le rite d'ancrage du Centre Sri Aurobindo de Montréal fut à mon sens l'installation en 1987 d'un monument dans la salle de méditation contenant un reliquaire de Sri Aurobindo. En 1987, le vice-président du Centre Sri Aurobindo de l'époque, Christian Feuillette, en accord avec la présidente Madeleine Gosselin, demanda à l'Ashram de Sri Aurobindo à Pondichéry de pouvoir installer des reliques de Sri Aurobindo à Montréal. Il partit à Pondichéry une fois sa demande acceptée et les membres du Centre préparèrent pendant ce temps la réception des reliques à Montréal. Ce rite fut signifiant non seulement par la portée symbolique de cet acte, avec la présence de reliques de Sri Aurobindo à Montréal, mais aussi - et peut-être surtout - par la mise en ouvre collective que ce rite a supposée et impliquée. La valeur symbolique de ce rituel d'ancrage ${ }^{15}$ a pu être de manifester la capacité de la communauté du Centre Sri Aurobindo de vivre et de forger son identité propre en faisant face à la mort (de la Mère en 1973 et de Sri Aurobindo en 1950). Un extrait d'un message distribué en 2017 par le Centre Sri Aurobindo interprète ainsi la signification de cet événement :

« Ces reliques [de Sri Aurobindo] sont enchâssées dans un reliquaire, ou Samadhi, moins pour perpétuer la mémoire de ces grands êtres que pour concrétiser leur Présence vivante et garder agissante la Force Divine qu'ils ont incarnée. Ainsi c'est la présence même de Sri Aurobindo qui émane de ses reliques.

13 Voir par exemple un de ses messages pour Auroville : «Programme : la recherche à travers l'expérience de la vérité suprême, une vie divine, mais pas de religions » (La Mère, 1991).

14 Marc Bradley utilise ce concept en interprétant l'implantation sur le long terme de la communauté tamoule au Canada à partir de l'élaboration de ses rites funéraires. Je reprends ce concept en gardant juste son idée de volonté de pérennité et de construction identitaire tandis que la communauté concernée est principalement québécoise.

15 La valeur symbolique du «rituel d'ancrage» dans le cas de la communauté tamoule étudiée par Marc Bradley résidait notamment dans sa capacité à établir de nouveaux rites funéraires, ce qui est aussi une façon de se redéfinir en tant que vivants faisant face à la mort. 


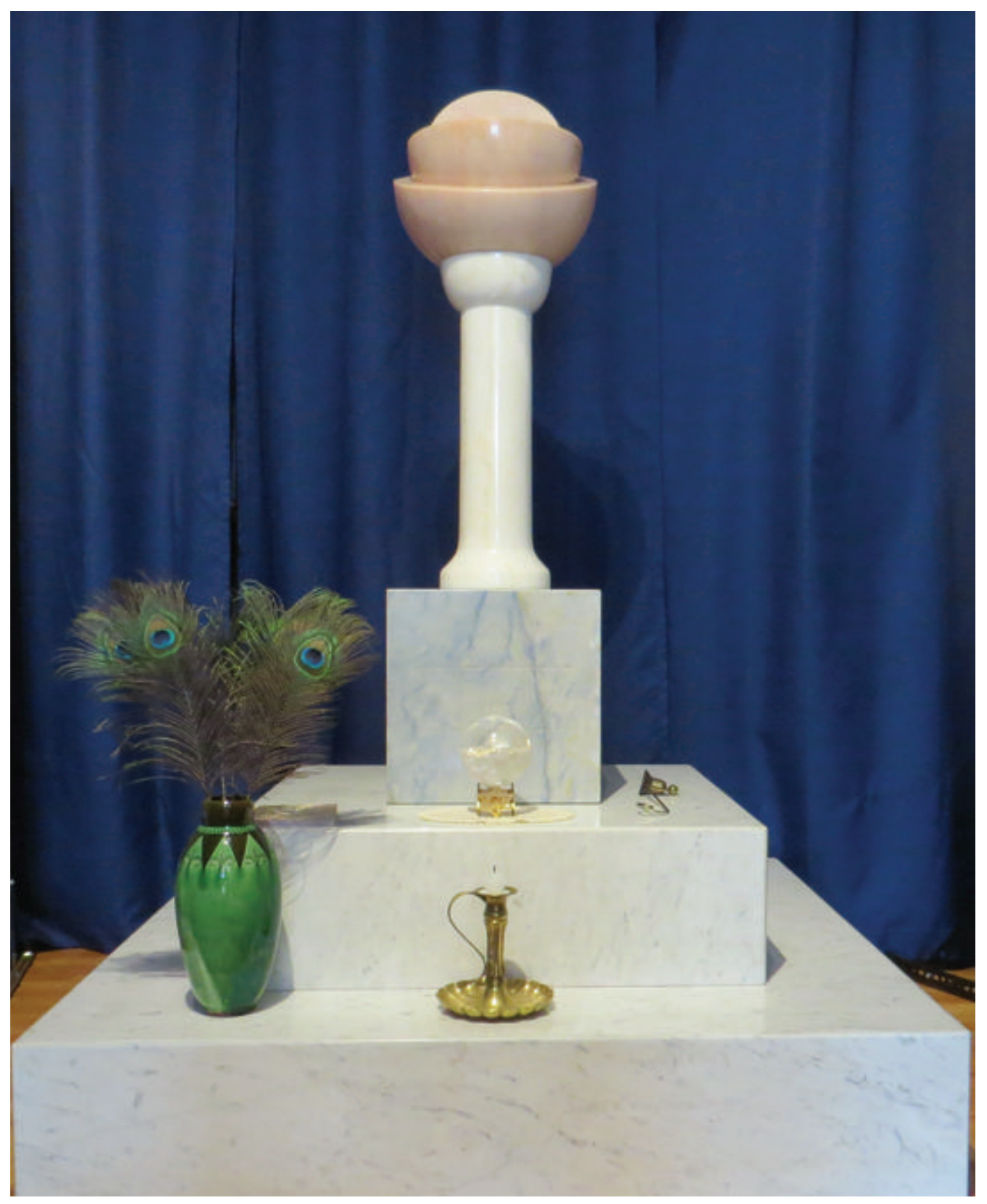

Le reliquaire de Sri Aurobindo au Centre de Montréal.

Ces fragments d'ongles et de cheveux du maître de Pondichéry transportent et rayonnent son atmosphère spirituelle [...]. C'est la première fois qu'un centre Sri Aurobindo situé en dehors de l'Inde reçoit cet insigne honneur. »

Les fragments d'ongles et de cheveux ont été recueillis du vivant de Sri Aurobindo par des disciples responsables de sa toilette, lorsque ce dernier n'était plus en état de la faire lui-même, un de ces disciples ayant pu d'ailleurs être
Champaklal. D'ailleurs, en 1988, la visite de Champaklal (1903-1992), peintre, yogi et un des principaux disciples de la Mère et de Sri Aurobindo pendant plus de cinquante ans, fut aussi l'occasion de sa reconnaissance physique du Centre Sri Aurobindo de Montréal en tant qu'un des plus éminents représentants de l'Ashram Sri Aurobindo de Pondichéry. Le même message du Centre Sri Aurobindo au sujet du reliquaire précise ensuite que ces reliques s'enchâssent de l'extérieur vers l'intérieur dans 
six cubes façonnés en marbre bleu, en bois de rose, en bois de tek, en bois de santal, en argent et finalement en or. Ce cube de marbre bleu posé sur deux grandes marches de marbre blanc est surmonté « d'une colonne de marbre blanc soutenant un lotus rose, symbole du Maître » d'environ un mètre cinquante de hauteur réalisée par l'artiste québécois Carlos Calado, selon le même message et comme la photo ciaprès le montre.

L'enchâssement même de reliques de Sri Aurobindo dans une œuvre artistique québécoise manifeste matériellement l'effort d'une synthèse hybridant culturellement une sacralité héritée de rites hindous de dévotion posthume (Aymard, 2009), une reconfiguration de cet hindouisme traditionnel entre une perspective propre à Sri Aurobindo et la Mère intégrant une vision évolutionniste tournée vers l'avenir de l'Inde et du monde, et une culture québécoise en pleine émergence artistique après la Révolution tranquille.

La présence des reliques de Sri Aurobindo manifeste à la fois la volonté affichée de centres internationaux de se relier à la réalisation physique opérée par le yogi dans son corps ou ses restes ${ }^{16}$ et leur capacité à créer un espace sacralisé dupliquant en partie celui du Samadhi de l'Ashram de Pondichéry. Les centres internationaux recueillant des reliques de Sri Aurobindo sont notamment- outre le Centre Sri Aurobindo de Montréal - un Ashram de Sri Aurobindo en Californie, un centre en Floride, un à Londres et un à Nairobi au Kenya. À l'instar de la dispersion du corps de la déesse Satī par Shiva en Inde créant en chaque lieu un espace sacré particulier (un pitha), la dissémination même du corps de Sri Aurobindo dans le monde peut s'interpréter comme sacralisant ces espaces particuliers à titre de lieux de culte posthumes. D'un autre point de vue, cette dissémination corporelle symbolique est aussi une
« dissémiNation »(Bhabha, 2007) qui déconstruit le caractère identitaire national indien en s'hybridant aux autres cultures, ce qui souligne en retour la dimension transnationale du mouvement spirituel Mère-Sri Aurobindo.

16 Il faut rappeler qu'en Inde, dans l'hindouisme, tout ce qui provient du corps tel que cheveux, ongles ou autres matières organiques est considéré comme impur... sauf parfois dans le cas de yogis réputés comme ayant dépassé cette impureté. 


\title{
5 Rituels et foi au cœur d'une utopie :
}

\section{oscillations et négociations}

\section{autour des pratiques croyantes à Auroville}

\section{Marie Horassius}

\begin{abstract}
"Que nous soyons célèbres ou inconnus n'a absolument aucune importance du point de vue spirituel. Ce n'est que de l'esprit propagandiste. Nous ne sommes ni un parti politique, ni une Église, ni une religion à la recherche d'adhérents ou de prosélytes. Un seul homme qui poursuit sérieusement le yoga a plus de valeur que mille hommes célèbres. »
\end{abstract}

Sri Aurobindo (1997a)

\subsection{Présentation d'Auroville et de son étude}

Auroville est une communauté internationale, située dans l'État du Tamil Nadu, en Inde du Sud. Ce territoire de 19,63 km² (Meier, 2006), proche de Pondichéry (12 km), a été fondé en 1968 sous l'égide de l'UNESCO et du gouvernement central de l'Inde. Son ambition à l'époque était de devenir un «laboratoire d'expérimentation pour la construction de l'Unité Humaine » ou, selon les termes du fondateur, d'une "Unité dans la diversité » (Sri Aurobindo. 1972 b). Elle compte aujourd'hui 2852 habitants (dont 697 enfants) de 56 nationalités différentes ${ }^{1}$.

Deux grandes figures sont à l'origine du projet d'Auroville, la Mère et Sri Aurobindo. Auroville a été étudiée sous de nombreux angles universitaires. Les thématiques privilégiées sont les champs de recherche où la communauté est la plus active : l'écologie, l'éducation et l'architecture. En 1993 fut publiée une des premières études universitaires sur Auroville. Stuart R. Leard, sociologue canadien, menait des recherches sur la communauté depuis 1982, soit quatorze ans après sa fondation. Plus de dix ans plus tard, il publia son master de recherche : The Routinization of Charisma (Leard, 1993). C'est un des rares documents traitant spécifiquement des pratiques autour de la foi à Auroville. S. Leard analyse, de 1981 à 1988, la manière dont les Auroviliens $^{2}$ "résistèrent » après le décès de « la Mère » à ce que l'on nomme en sciences humaines l'institutionnalisation de leur foi.

Cet article cherche à mettre en perspective le travail de Stuart Leard, avec nos propres

1 http://www.auroville.org/, recensement du $1^{\mathrm{er}}$ mai 2018.

2 Aurovilien s'écrit avec un seul « 1 », selon la volonté de La Mère rapportée dans l'avant-propos du livre de Jean Laroquette : Auroville un aller simple?, présentée aussi dans Mère parle d'Auroville : " Pour être un Vrai Aurovilien », (La Mère, 1991: 31). 
analyses effectuées vingt ans plus tard durant la période de 2011 à 2018. À l'instar de l'anthropologue canadien, observe-t-on aujourd'hui à Auroville une institutionnalisation de la foi ? Ce phénomène d'institutionnalisation, théorisé par Max Weber (1971 : 320) dans Économie et Société, essai repris dans Sociologie des religions, est - selon l'auteur - la résultante logique, lorsqu'un groupe fondé autour d'une figure charismatique se structure et se transforme en religion. Toutefois, en 1988, Auroville et ses résidents disaient rejeter toute croyance dogmatique et refuser de transformer leur foi en religion. Telles étaient en tout cas les conclusions de Leard, en observant la résistance $\mathrm{du} \quad \mathrm{groupe} \quad \mathrm{a} u \quad \mathrm{phé}$ no mène

\subsubsection{Routinisation et charisme}

Dans un premier temps, Stuart Leard replace son sujet et élabore une analyse épistémologique de la notion de charisme et de routinisation. La "routinisation » ou « quotidianisation » (selon la traduction des éditions Champs-Flammarion, éditée en 2013) du charisme est une notion développée par Max Weber dans Économie et Société. Le mot kharisma désigne en grec la grâce ou la faveur attachée à une personne ou à un acte. Weber en donne cette définition dans Économie et Société :

« Nous appellerons charisme la qualité extraordinaire (...) d'un personnage qui est considéré comme doué de forces et de qualités surnaturelles ou surhumaines, ou au moins spécifiquement extraquotidiennes (extra-mondaines) qui ne sont pas accessibles à tous, ou comme envoyé par Dieu, ou comme exemplaire, et qui pour cette raison est considéré comme “chef”.» (Weber, 1971 : 320)

La notion de quotidianisation est le phénomène qui amène les dévots d'un prophète (ou de la figure charismatique) à mettre en place un ordre social et moral autour des valeurs inculquées par le fondateur - et particulièrement la transformation progressive d'institutionnalisation. Vingt ans plus tard, ces conclusions demeurent-elles pertinentes?

Après avoir présenté brièvement son étude et rappelé les définitions des termes utilisés par Max Weber, nous tenterons d'analyser les différents événements historiques qui ont conduit les Auroviliens à rejeter la construction de dogmes autour de leurs croyances. Par la suite, nous présenterons les différents rituels collectifs et individuels existant à Auroville. Ces exemples donneront à penser sur les pratiques autour du croire spécifique à cet espace. Enfin, nous conclurons en nous demandant si ces " négociations et oscillations » permettent de «croire sans religion ».

de l'autorité charismatique en ce qu'il nomme un "charisme de fonction » (celui des prêtres et officiants en général). Au décès (ou lors du départ) du prophète, les adeptes cherchent une manière de mettre en pratique ces valeurs dans la quotidienneté de la vie:

« La transformation du groupe des adeptes personnels du prophète en une communauté est, par conséquent, la forme normale qu'adopte la doctrine prophétique quand elle se quotidianise : celle-ci remplit dès lors une fonction dans une institution permanente. » (Weber, $2013:$ 178)

La foi à Auroville serait en même temps celle d'une religiosité éthique, donc orientée dans le monde, celle d'une recherche de la délivrance par l'union au divin (religion du salut) et évidemment, à ses débuts, une religiosité " mystico-magique " liée au charisme des fondateurs. Ces éléments s'entrecroisent.

Leard conclut par l'analyse des résistances à une dogmatisation du " croire » à Auroville en s'appuyant sur la forme de la foi des résidents, qu'il définit comme "mysticisme intramondain ». En effet, il n'y a ni guru à Auroville, ni rituels institués comme tels, ni prières collectives 
ou rassemblements obligatoires. Rien n'est obligatoire à Auroville (sauf le respect des lois indiennes) et il n'y a pas de forces coercitives contrôlant sa population (police, armée, huissier..... ${ }^{3}$

Dans les faits, Auroville construit son idéal, ses formes du croire autour d'une forme " nébuleuse » (Champion, 1990, 2007 ; Garnoussi, 2007). Il y a de grandes lignes directrices, des valeurs, des perspectives communes, mais pas de ritualisation de la foi. Il n'y a que peu de traces d'un croire partagé, et la priorité est laissée à la recherche d'un « développement personnel ». Cela correspond finalement à la définition d'une foi intramondaine décrite par Weber dans Sociologie des Religions et reprise par Leard. Dans sa mise en pratique, elle n'est ni liée à l'ascétisme ni à une recherche d'un retrait de la vie mondaine, car elle est orientée dans l'ici-bas (hic et nunc) ; pourtant, elle se réfère et s'associe à des méthodes liées à l'hindouisme qui valorisent, quant à elle, ces démarches ${ }^{4}$.

Nous avons également observé sur place de nombreuses résistances, des tensions parfois, afin de garder la "parole juste » face à sa foi à Auroville. Oscillant de manière constante entre les rappels de leur guru par les citations et un rejet du religieux dogmatique, les Auroviliens passent sans cesse du pragmatisme matérialiste le plus radical à des rituels ou des gestes dévotionnels traditionnels (culte de l'image, sacralité des livres, prières, chants...).

À l'aide d'exemples concrets de ces « négociations du réel » (Piette, 2003), nous montrerons la nécessité de prendre en compte les postures des individus, leurs interprétations, mais aussi leurs gestes. En saisissant leur manière de percevoir le monde et leur place dans celui-ci, nous proposerons une perspective plus complète de cet espace et de ses résidents.

\subsubsection{L'indépendance d'Auroville contre la Sri Aurobindo Society}

Cet événement crucial dans l'histoire de la ville est souvent décrit par les pionniers en termes épiques, comme une grande lutte pour la «Vérité » (Satprem, 1973). C'est d'autant plus intéressant que, selon le modèle de Weber, c'est au travers de la formation "en communauté » que les adeptes d'un guru institutionnalisent leur foi. Le rejet de l'institutionnalisation est à l'origine de la scission des deux entités. La Mère avait délégué depuis 1966 de nombreuses tâches à des dévots de l'Ashram. Quelques figures sont citées régulièrement par les Auroviliens (ou Satprem) comme les ennemis de "la Vérité ». Ainsi, Navajata (qui était le trésorier et le secrétaire général de la Sri Aurobindo Society) est accusé d'avoir voulu transformer Auroville et la métaphysique de la Mère et de Sri Aurobindo en une religion. Cela a été perçu par les Auroviliens comme une trahison, au regard des propos des fondateurs :

3 À la question : "Auroville est-elle une secte pour vous ? », l'ancien premier ministre de l'Inde, M. Singh, répondit : «Dans une secte c'est facile d'y entrer, mais très difficile d'en sortir. C'est l'inverse à Auroville, c'est difficile d'y entrer, mais très facile d'en partir. » Propos recueillis en juillet 2012 auprès du Outreach Media d'Auroville. Conversation informelle de Manmohan Singh avec des Auroviliens (30 juin 2012).

4 C'est pourquoi la définition de Luc Boltanski et Eve Chiapello (1999) d'un «néo-personnalisme » où l'accent est mis sur la recherche individuelle d'un sens, voire celle d'un "individualisme-subjectiviste » (par opposition à l'« individualisme possessif » de Macpherson [1962]), est selon nous plus proche de ce qui se passe dans la réalité. En effet, l'une des valeurs communes distinctives d'Auroville est son attachement à la notion de karma yoga: le yoga de l'action, la dévotion par l'acte. Il y a certes une recherche de purification et de libération par la recherche du Divin ( «ers le haut »), mais la base du travail « est de faire descendre le Divin dans la matière ». Il y a ainsi double mouvement : shiva-shakti, purusha-prakriti, écrit-on en sanskrit. 
« Auroville est pour ceux qui veulent vivre une vie essentiellement divine, mais qui renoncent à toutes les religions, qu'elles soient anciennes, modernes ou futures.(La Mère, 1991 : 36)

Je dois dire qu'il est loin de mon objectif de propager quelque religion que ce soit, nouvelle ou ancienne, pour l'humanité future. Un chemin à ouvrir qui est encore bloqué et non une religion à fonder est ma conception en la matière. » (Sri Aurobindo, 1972 c : 139).

Plus que métaphysique ou moral, l'enjeu de cette qualification était aussi de nature juridique, légale et politique. ${ }^{5}$ En effet, la Constitution indienne garantit une indépendance des ashrams à l'égard de l'État. Lorsqu'en 1980 Indira Gandhi émit une ordonnance provisoire de mise sous tutelle d'Auroville par l'État, la Sri Aurobindo Society s'empressa de porter l'affaire devant la Cour de Justice, déclarant qu'Auroville était une religion ; l'État n'aurait pas alors eu le droit de s'ingérer dans le litige entre la Sri Aurobindo Society (SAS) et Auroville. En 1982, la Haute Cour de Justice conclut finalement qu'Auroville n'était pas une religion ${ }^{6}$. La Cour maintint le statut spécifique d'Auroville sous la tutelle de l'État central de l'Inde. Par ce statut, Auroville obtint son indépendance et le gouvernement put octroyer à la communauté des financements, notamment dans le domaine de l'éducation et de la recherche dans le cadre des services du ministère des Ressources Humaines et du Développement (HRD). Cependant, l'ordonnance de 1980 était temporaire. En 1988, Kireet Joshi (ancien secrétaire général au ministère de l'Éducation sous Indira Gandhi) soumit au Parlement indien le projet de l'Acte de Fondation d'Auroville, approuvé et toujours en vigueur. Ainsi, Auroville, accréditée par le gouvernement, put gérer par elle-même l'obtention des visas (sous la tutelle du Collector [Préfet]), ses fonds, l'achat des terres et la répartition de l'argent. Le système continue de fonctionner selon ce principe : l'État supervise les demandes de visas, il verse une aide à hauteur de 50000 dollars par mois à Auroville pour la création d'infrastructures et pour la recherche scientifique et éducative. Largent est ensuite distribué sous l'égide du BCC (Budget Coordination Committee), un groupe soit élu parmi une liste de candidats, soit — pendant quelque temps - tiré au sort parmi une liste de doyens. Depuis juillet 2016, un nouveau mandat restructurant l'ensemble des comités valorise à nouveau un système rotatif et soumet les volontaires à une demande d'approbation auprès des résidents par l'intermédiaire de l'Auronet et du journal hebdomadaire News\&Notes?

\footnotetext{
5 La Cour Suprême de Justice de l'Inde trancha pour le caractère a-religieux de la philosophie de Sri Aurobindo et, de ce fait, homologua l'indépendance de la ville à l'égard de l'Ashram (Minor, 1999). Robert Minor a fait une longue analyse des enjeux philosophiques et juridiques de cette désignation. À l'instar de Robert Minor, David Brême (2016) a fait une analyse dans Religiologiques sur les nombreux enjeux politiques et religieux sous-jacents à cette désignation.

6 «In 1980, responding to requests from the residents, the Government of India passed the Auroville Emergency Provisions Act, whereby the management of all assets and undertakings relatable to Auroville were, for a limited period of time, vested in the Government of India. The Sri Aurobindo Society subsequently challenged the constitutional validity of the Act, on the main ground that Auroville was a religious denomination and that the Government had violated the Indian Constitution by passing the Act. In November 1982 the Constitution Bench of the Supreme Court of India judged that Auroville did not constitute a religious denomination, and that the teachings of Sri Aurobindo only represent his philosophy and not a religion. The validity of the Act was upheld. » http:// www.auroville.org/organisation/org_gov_india.htm
}

7 Les deux médias internes de la communauté. Auronet est l'intranet de la communauté et le News and Notes son journal hebdomadaire. 
En analysant ces événements, Stuart Leard conclut qu'il y eut une résistance contre une institutionnalisation de la foi à Auroville. Le principe dicté par les fondateurs d'une spiritualité intramondaine - mélangeant exercices spécifiques yogiques, vertus éthiques, recherches esthétiques et action désintéressée dans le monde - laisse beaucoup de place à une expression «nébuleuse » des pratiques du croire. Celles-ci sont liées à la subjectivité individuelle et à l'expérience intime du Divin. Paul Heelas, dans son ouvrage sur le New Age Movement (1996), explique, à l'instar de Nadia Garnoussi (2007), que cette nébuleuse des pratiques orientées sur une vision individualiste, subjective, mais interconnectée des individus, peut parfois être portée par des figures charismatiques, mais relève malgré tout d'un principe non dogmatique et non institutionnel. Comme il est écrit dans la Bhagavad-Gìtā (un texte fondateur de l'hindouisme et pour Sri Aurobindo) :

« Mieux vaut [pour chacun] suivre sa propre loi d'action, même imparfaite, que la loi d'autrui, même bien appliquée.

Mieux vaut périr dans sa propre loi ; il est périlleux de suivre la loi d'autrui. » (Bhagavad-Gìtā, 1991: 2)

Paul Heelas écrit ainsi : "Une orientation mystique intramondaine est caractérisée par la dévotion individuelle double au développement intérieur et à l'engagement social. » (1996:4) Rien de surprenant de voir les Auroviliens associer ces deux postures : cela entre pleinement dans les pratiques de foi contemporaines.

Au regard de la recherche de Leard, nous développerons trois points issus de nos propres données empiriques, qui font ressortir un phénomène de "routinisation » ou tout du moins une institutionnalisation implicite des pratiques dévotionnelles à Auroville. En prenant en compte les analyses de Paul Heelas sur le New Age et de Paul H. Ray et Sherry Ruth Anderson sur les Créatifs culturels (2000), nous ferons la part entre les croyances proprement auroviliennes avec leurs mises en scène, et un recensement des pratiques du croire à Auroville, couvrant une palette de possibilités larges : de l'hindouisme au New Age, en passant par le shaktisme jusqu'à un athéisme radical.

\subsection{L'institutionnalisation « psychologique » du croire}

Le premier point est ce que nous appellerons « l'institutionnalisation psychologique » du croire. Auroville demande à ses ressortissants un effort de "narrativité ». Paul Ricœur définit l'identité narrative en ces termes : la capacité de la personne à mettre en récit de manière concordante les évènements de son existence (1990:149).

Lorsque l'on arrive à Auroville, on est confronté à cette mise en récit de soi. Tout d'abord par les questions quotidiennes des Auroviliens et des touristes de passage : «D'où tu viens ? Pourquoi es-tu là ? ». Ensuite, en faisant la démarche pour obtenir le statut d'Aurovilien : on passe alors par un processus d'intégration du bon discours. Un comité va convoquer la personne pour lui demander ses intentions et son histoire. Enfin, après une période d'un an et l'acceptation par les résidents, on accède ou non au statut d'Aurovilien. Ces processus contraignent les individus à rationaliser leur choix et leur trajectoire de vie et créent, dès lors, une " communion d'idée ». Ces grands schèmes communs seront alors constitutifs d'une « identité collective commune »8.

Ces contingences vont entraîner l'émergence d'objets médiateurs qui légitiment la «bonne foi $»^{9}$ à Auroville. Une majorité des

8 Voir les analyses en sociologie de Jane Meier (2006) sur cette question.

9 On entend « bonne foi » dans le sens de la « manière appropriée d'exprimer sa foi, sa dévotion ». 
Auroviliens reconnaît une grande valeur à la pensée des fondateurs. Beaucoup usent de leurs expressions (" Mère ou Sri Aurobindo a dit»); même les «athées » mentionnent leurs pensées pour interroger les idéaux de vie commune ou plus simplement lors de discussions. Toutefois, les paroles de la Mère ou de Sri Aurobindo sont souvent (si ce n'est quasi systématiquement) utilisées en qualité de figures d'autorité : on fait appel aux principes jugés supérieurs pour " moraliser " des discours techniques et/ou politiques.

On peut observer des panneaux disséminés partout dans la ville où des mots de la Mère sont reproduits. Sa signature et ses bénédictions ornent les maisons, les entreprises et les magasins. Les photographies de la Mère et de Sri Aurobindo immortalisent dans la pensée des résidents le projet que les fondateurs avaient imaginé. Ces messages dévoilés sont en quelque sorte des rappels à l'ordre ou des remémorations, car les «Auroviliens sont là pour quelque chose » : « on travaille à plus grand que soi », m'a expliqué une entrepreneuse aurovilienne.

Un exemple flagrant est celui de la Solar Kitchen ${ }^{10}$, la cantine de la ville qui sert près de 1000 repas par jour. C'est un lieu de rassemblement courtois en plus d'être utilitaire. On observe une répartition spatiale spécifique des résidents, et une ségrégation temporelle (les guests sont priés de venir à partir d'une heure de l'après-midi) pour raccourcir la file d'attente (afin que les Auroviliens puissent « retourner travailler en temps et en heure »). Au moment de se servir, juste au-dessus des plateaux, un panneau d'un mètre d'envergure reproduit l'écriture de la Mère et ses paroles :
"Auroville n'est pas fait pour la satisfaction des désirs, mais pour la croissance de la vraie conscience. " (La Mère, $1991:$ 33) ${ }^{11}$

Cette parole sacrée est utilisée pour « raisonner » les touristes et les Auroviliens, pour leur rappeler qu'il ne faut pas gâcher la nourriture (au cas où on passerait à côté, un panneau avec le poids des déchets de la veille rappelle qu'il n'est pas bon de gaspiller), et que le Yoga Intégral est une discipline constante.

On l'évoque aussi lors des meetings : chaque fois, on présente l'idéal énoncé, puis on débat pour savoir comment l'harmoniser avec la réalité du terrain. Deux courants alors s'opposent : ceux, fervents dévots, qui expliquent que la Mère a «vu » Auroville, mais que Sa vision est trop " haute » pour qu'on puisse la comprendre à notre échelle et les autres, qui expliquent que la Mère a toujours tenu un discours dynamique, organique, car « le vivant change, le mort est figé ». Ainsi, la ville devrait calquer ses recherches et infrastructures sur cette idée de changement. C'est plutôt le camp des dévots qui remporte la partie. Au pire, ils trouvent une justification a posteriori. Lorsque fut abordée la question de savoir s'il fallait ou non créer un lac autour du Matrimandir, selon le plan de Roger Anger (validé par la Mère), le débat fut houleux. Aujourd'hui après des années d'études géologiques, hydrologiques et autres, l'un des agents principaux responsables de cette question répond dans son interview :

« Nous arrivons doucement au point où nous pouvons voir la sagesse des mots de Mère. C'était difficile de le voir avant parce que parler d'un lac était un peu de la science-fiction dans la situation dans

10 La sociologue Shanti Pillai a travaillé sur ces approches dans le courant des Performances Studies. Lors de la rédaction de cet article, nous n’avions pas encore eu accès au document ; pour pallier ces manques, nous intégrerons dans le corps du texte les références liées à sa thèse de 2005. L'auteure aborde toutefois ces thématiques dans sa perspective et nous ne reprenons pas l'ensemble de ses interprétations. (Pillai, 2005 : 147-148)

11 Cette citation provient d'un recueil non publié Bénédiction à Auroville, ce mot a été donné par la Mère le 16 juin 1968. 
laquelle nous étions. Mais cela fait tellement plus de sens ce qu'elle a dit, d'avoir un lac et un approvisionnement d'eau pour Auroville, bien plus que seulement des puits. » (Tency, Belge arrivé en 1969 à 21 ans, resté vivre à partir de 1971) (Narad, 2016)

\subsection{Rituel collectif du Bonfire}

Le bonfire est à l'origine un mot dérivé du français : «bon feu », qui renvoie feux de joie de la Saint-Jean ${ }^{12}$. Il y a deux bonfires par an à Auroville13 : pour l'anniversaire d'Auroville, le 28 février, et pour l'anniversaire de Sri Aurobindo (qui est aussi la fête d'indépendance de l'Inde), le 15 août. Pour l'anniversaire de la Mère le 21 février, il n'y a pas de grands événements : simplement une méditation collective à l'amphithéâtre (qui n'attire pas beaucoup de monde), les dévots ( $y$ compris auroviliens) se rendant pour leur part au Samadhit (Pondichéry) pour y rendre hommage à la Mère. C'est alors plus un rituel individuel (« intime » m’a-t-on dit) qu'une célébration collective.

Les bonfires consistent en des rencontres matinales ( $4 \mathrm{~h} \mathrm{du}$ matin) pour une méditation/ rencontre silencieuse. Assis dans l'amphithéâtre près du Matrimandir, on allume un grand feu avant le lever du soleil vers $5 \mathrm{~h}$. Sur une bande sonore est diffusé soit un texte de la Mère pour le 28 février, soit un texte d'Aurobindo le 15 août. La Charte est toujours diffusée auparavant dans l'enregistrement original où l'on entend la Mère déclamer les quatre points constitutifs de la
On observe alors que les paroles des fondateurs sont de l'ordre du sacré. Guides de vie, rappels à l'ordre, elles parsèment la vie des Auroviliens, qu'ils en aient conscience ou pas, qu'ils le désirent ou non. À l'instar de ces paroles rappelant le sacré, nous allons maintenant tenter d'identifier les rituels présents à Auroville.

fondation d'Auroville en 1968. Souvent, lors de la réunion du 28 février, est lu soit Un Rêve ${ }^{15}$ (La Mère, 1991 :7), soit La Grande Aventure (La Mère, 1997 :144-51), qui correspondent à « l'appel aux œuvres ». Le 15 août, c'est plutôt un texte d'Aurobindo issu de Savitri (1950), du Cycle Humain (1972a) ou des Letters on Yoga (1972c), plus accessibles au grand public. C'est parfois même un moment de liberté pour la contestation politique. Lors de mes observations en 2012, 2014 et 2015, le texte choisi ne semblait pas anodin. Il était en lien directement avec les conflits politiques internes du moment. Par ce texte, on rappelle alors le «bon comportement » qu'un Aurovilien devrait avoir et quelle attitude est attendue des aspirants au Yoga Intégral de Sri Aurobindo. Le soleil levé, les participants se lèvent aussi, discutent un peu, saluent les présents. Certains se félicitent d'être venus. C'est aussi un moment de paix autour d'une marque de dévotion avec les ashramites, reconnaissables, car ils sont vêtus tout en blanc.

Mircea Eliade explique selon nous dans Le Sacré et Le Profane ce qui est célébré :

12 Shanti Pillai (2005) explique dans sa thèse que cela pourrait éventuellement venir aussi des feux crématoires indiens et donc de «Bones Fires » : les feux d'os. C'est la seule personne, à notre connaissance, à mentionner cette interprétation.

13 Officiellement, il y en a un troisième, lors du Nouvel An, mais je n'y ai pas personnellement assisté, et mes interlocuteurs n'en ont pas fait mention, et encore moins, comme d'un « événement » unitaire (à la différence de l'anniversaire d'Auroville).

${ }^{14} \mathrm{Cf}$. article de Patrick Beldio

15 «Un Rêve » est un texte écrit par la mère en 1954, elle le dédia à Auroville peu après son inauguration. Avec $L a$ Charte, Un Rêve (La Mère, 1991 : 7) est un résumé des grandes aspirations et valeurs de la communauté. 
« Toute fête religieuse, tout Temps liturgique consiste dans la réactualisation d'un évènement sacré qui a eu lieu dans un passé mythique, "au commencement ». Participer religieusement à une fête implique que l'on sort de la durée temporelle " ordinaire " pour réintégrer le Temps mythique réactualisé par la fête même. »(Eliade, 1957 : 63)

Les symboles, à l'instar du reste de la communauté, sont discrets, il n'y a rien d'ostentatoire, tout est dans la "sobriété » du signifiant et dans la continuité du geste. Et c'est justement là que les paroles de Mircea Eliade résonnent d'autant plus : on rejoue le Temps $d u$ commencement. Chaque année, deux fois par an, les pionniers revivent l'inauguration de la ville ${ }^{16}$,

\subsection{Le Matrimandir}

Le Matrimandir19 (le Temple / la maison de la Mère [Matri : Mère, Mandir : le temple] en sanskrit) est un monument intéressant à de nombreux égards, notamment pour sa

\subsubsection{Matériellement}

Débuté au matin du 21 février 197120 , le Matrimandir a été achevé en 2008. Ses jardins sont toujours en construction. Par ces « grands travaux », la Mère entendait unir les Auroviliens revivent l'appel à " la grande Aventure » de la Mère en 1968. Toute la symbolique est explicite : on arrive la nuit pour repartir au soleil levant (à l'aurore : Auroville, la ville de l'Aurore). Le feu qui brûle dans l'obscurité : le feu de la foi, Agni ${ }^{17}$ porté par la parole des fondateurs et La Charte. $\mathrm{Au}$ commencement, il y eut le feu et l'appel : deux fois par an, les résidents renouvellent ce moment et scellent par là de nouveau le contrat/ l'alliance avec le Divin.

$\mathrm{Au}$ commencement, il y eut l'urne'18, puis le Matrimandir. Sur ce dernier point, on pourrait également mentionner les analyses d'Eliade sur l'espace sacré, pour parler du dernier « événement »/ « rituel » collectif, sur un point liminal, à la frontière entre le collectif et l'individuel : le Matrimandir.

symbolique. Il est ce que Mircea Eliade nomme un imago mundi (1957 : 43). Il (re)présente une image entière du monde symbolique, de la cosmogonie des Auroviliens.

autour d'un projet commun fondateur'21. Tous les pionniers y travaillèrent. Lors du coffrage, pour le troisième anniversaire d'Auroville, près du bonfire, chacun des participants déposa une

\footnotetext{
16 Ils revivent l'inauguration et la fin de l'excavation du Matrimandir en 1972. Le bonfire est une tradition qui date de 1971, absente en 1968. En 1971, il y eut trois bonfires sur le site. Toutefois, c'est en 1972 que les pionniers reproduisent le geste du «mélange des terres ».

17 « Par l'érection d'un autel de feu, Agni est rendu présent et la communication avec le monde des dieux est assurée. » (Eliade, $1957: 33$ )

18 L'urne est un réceptacle dans lequel plus de 147 poignées de terre provenant de 124 pays différents et de 23 États indiens ont été déposées lors de la fondation d'Auroville par des personnes du monde entier.
}

19 Cf. Pillai, 2005 : 327-352. Sur cette partie, il me semble que Shanti Pillai a beaucoup sur-interprété ses données, néanmoins ses observations restent intéressantes.

20 Le projet a été envisagé par la Mère dès 1969.

21 Ce qui, comme l'analyse David Brême (2017) dans sa thèse, participera même après sa mort à la construction d'un imaginaire collectif autour de la Mère et de la construction de ce qu'il nomme un « charisme communautaire». 
poignée de "gravillons dans la bétonnière "(Retournements, 2009 : 103) : : symbole de leur unité renouvelée. Lors de l'inauguration avec l'urne, les Auroviliens ont scellé de nouveau leur unité par un symbole areligieux, par un geste symbolique, et permettent aussi à ceux qui n'étaient pas là lors de l'inauguration de participer à ce symbole d'unité.

\subsubsection{Les symboles}

Symboliquement,le Matrimandir représente l'Euf Cosmique du mythe des origines védiques (l'œuf sorti de la mer de lait, qui donna le monde), m'a expliqué une architecte aurovilienne d'origine indienne. Il est au centre d'Auroville.

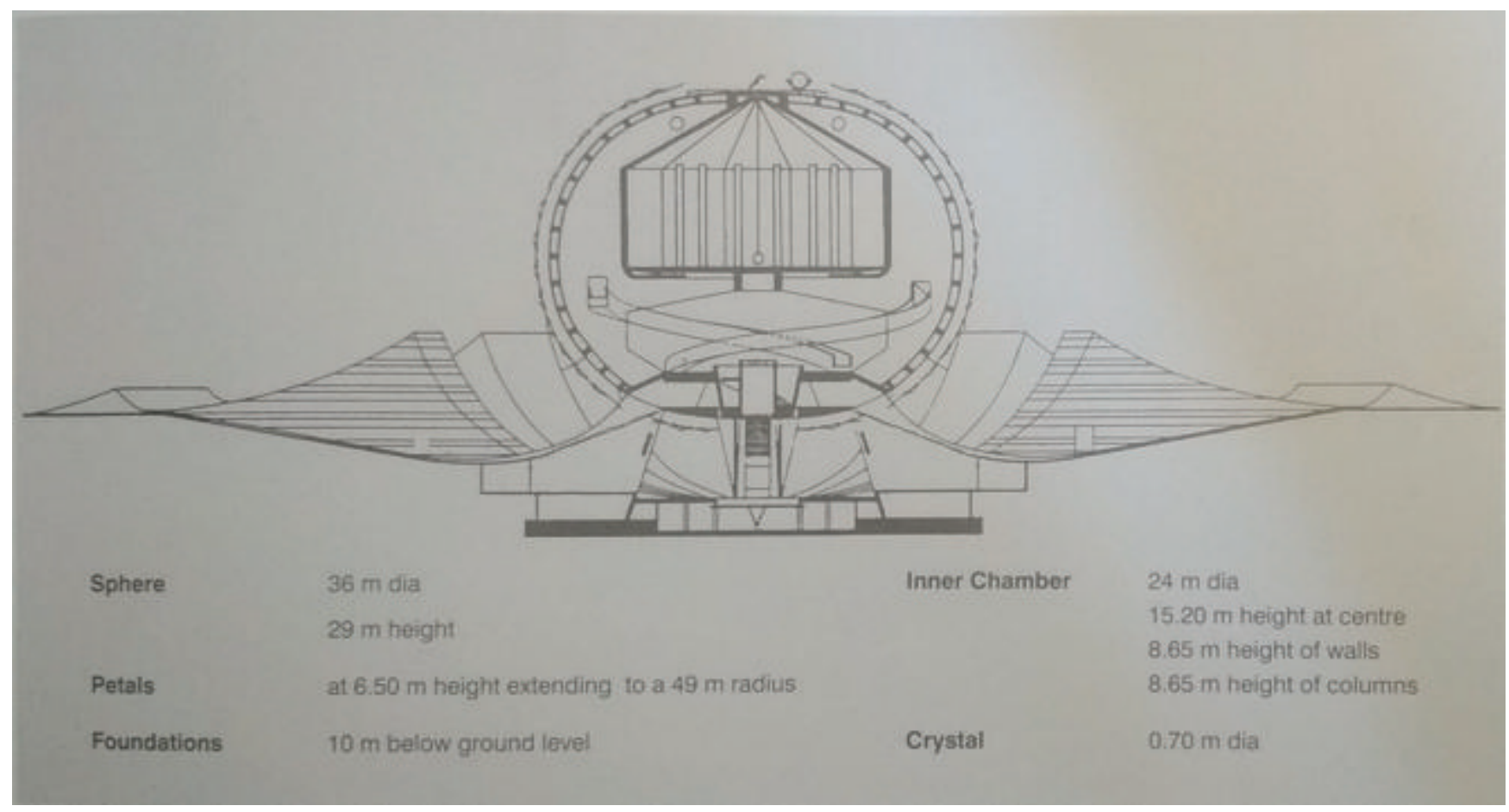

Il s'agit donc d'une sphère au centre de la ville qui se tient sur un disque, rappelant de nouveau l'œuf primordial. Le Matrimandir est surélevé : il est l'espace entre les niveaux cosmiques. Il ressemble aussi à une fleur, car la Mère chérissait les fleurs et symbolise "l'aspiration au soleil ». Entre les éléments tantrika et l'architecture futuriste ${ }^{22}$, il incarne symboliquement ce "pont entre le passé et l'avenir » décrit dans la Charte. Il est imago mundi par sa forme universelle géodésique.
Enfin, à l'intérieur, il est ouvert en son sommet pour laisser entrer une colonne de lumière qui traverse le Matrimandir jusqu'aux eaux tout en bas (Axis Mundi). Sa forme interne héliocentrique est une référence au Matérialisme Divin²3 de la Mère et à l'ADN. Sur ses parois se rencontrent des flèches montantes et descendantes, ce qui est un symbole shaktique. Le Matrimandir, dans son sobre décor, est riche en sens et en symboles. Il se laisse lire seulement pour ceux qui en font la démarche. Son style

22 Bientôt les portes, aujourd'hui battantes, s'ouvriront automatiquement par le bas (elles glisseront sous le Matrimandir, comme un vaisseau), m’a confié un des ingénieurs travaillant au Matrimandir en 2012.

23 La Mère : le Matérialisme Divin est un livre de Satprem publié en 1976 aux Éditions Laffont. Il retrace le travail que La Mère aurait cherché à faire à la fin de sa vie, auprès des cellules de son corps afin de les rendre « conscientes » et ainsi inverser le processus de la mort. 


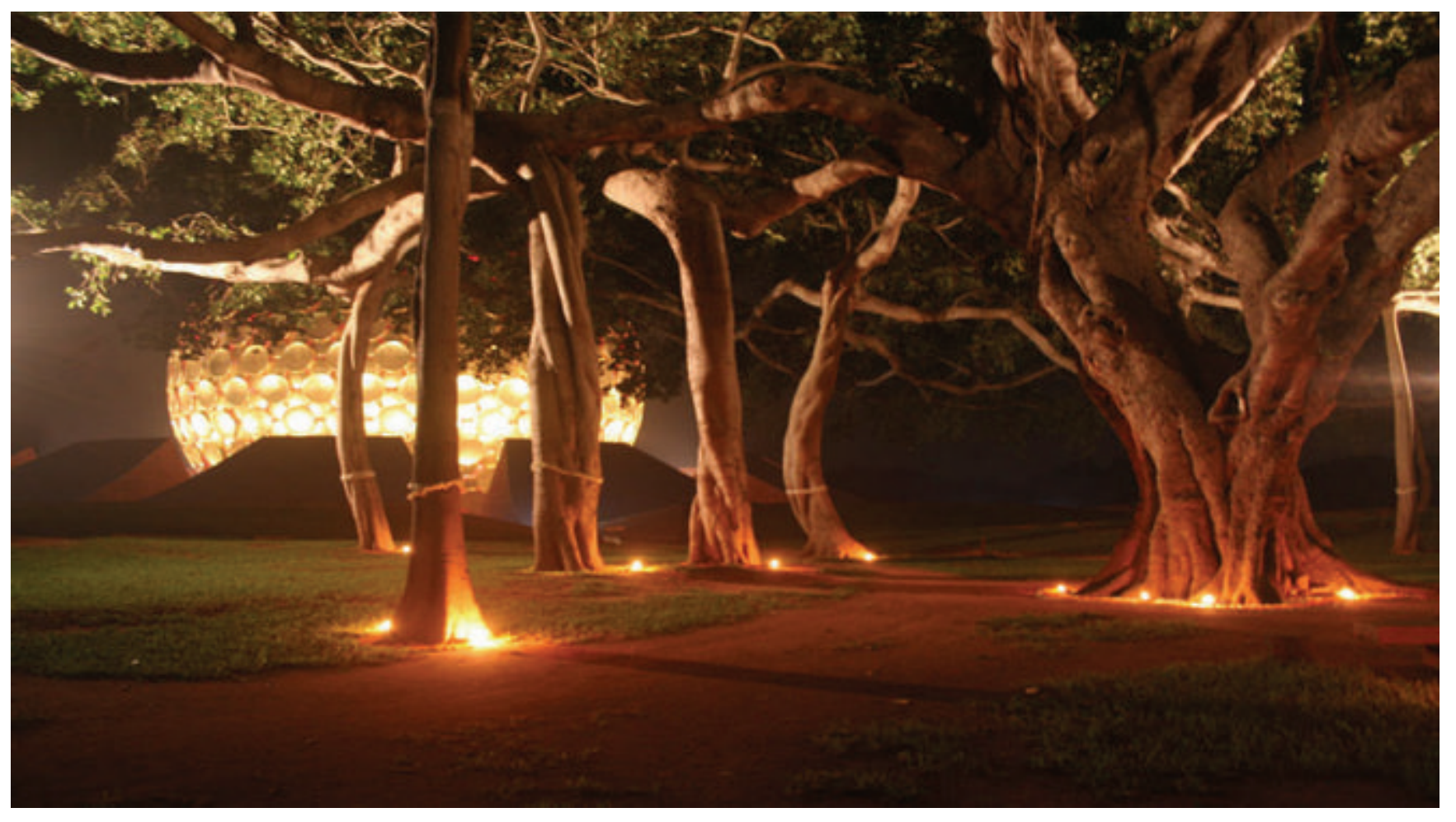

Le Banyan et le Matrimandir, photographie reproduite avec l'aimable autorisation de Auroville.org.

épuré laisse libre interprétation à l'observateur, auquel personne ne donnera d'explication. J'ai ainsi pu lire sur des blogues de voyage : la présence supposée d'étoiles de David, la Géode de Paris, une balle de golf géante, la Géode de Disney à San Francisco, une allégorie de la Terre, voire même un vaisseau extraterrestre... un cocon d'ésotérisme pur, incarné, mais pas nécessairement célébré.

C'est d'ailleurs en cela que le Matrimandir représente un espace liminaire du sacré entre collectif et individuel, parce qu'il n'est ni célébré ni consacré collectivement. Il est un espace collectif dédié à la recherche spirituelle individuelle par la méditation. Il n'y a pas de rite collectif en son sein. À l'intérieur, il $y$ a des symboles, mais pas d'images, pas de photographies. Il y a une flamme et de l'huile qui brûle, de l'eau descend des parois sur les symboles shaktiques. La paroi interne diffuse une lumière orangée telle la matrice maternelle (puisqu'on est dans le ventre du Temple de la Mère). Les rampes « élèvent » le visiteur jusqu'à la « chambre intérieure » blanche et vide. En son centre, un globe de cristal montre l'aspiration au « silence mental ». C'est un espace de recueillement qui symbolise l'unité aurovilienne par sa prestance. Mais par sa sobriété, il est aussi un rappel à l'humilité et au "devoir » (de concentration, de méditation...).

Enfin, des jardins " à la française » entourent le monument et le banyan, qui lui a aujourd'hui sa propre légende constitutive et fondatrice. Le banyan est le réel centre d'Auroville, le centre «vivant » choisi par la Mère : le premier habitant du plateau, dernier vestige d'un monde tropical disparu et premier témoin des efforts des Hommes pour le réhabiliter. 


\subsection{Les rituels individuels}

Les rituels individuels sont très sobres. Il est demandé de s'abstenir de ferveur débridée. Les Auroviliens refusent la citoyenneté

\subsubsection{Les signes d'appartenance}

Avec 56 nationalités différentes et plus de 1000 touristes par jour en moyenne (jusqu'à 10.000 en haute saison et 100 durant les périodes de mousson), il n'y a pas de type aurovilien. "Ce n'est pas écrit sur leur tête ", m'explique un résident en 2012. Parfois seulement, on distingue des petits signes d'appartenance, le plus souvent discrets. Les Auroviliens se reconnaissent plutôt à ce qu'ils portent : un médaillon, une bague avec le symbole de Mère ou d'Aurobindo. Les pionniers et les nouveaux arrivants portent régulièrement ces bijoux, les enfants d'Auroville beaucoup

\subsubsection{Les livres}

Un des éléments les plus marquants est le rapport des résidents aux livres. Tous ont une bibliothèque avec les livres de Sri Aurobindo, Satprem ou de la Mère (notamment L'Agenda de Mère).

J'ai pu voir à de nombreuses reprises une mise en scène "biblique » des livres : exposés sur un pupitre, ouverts sur une page au hasard, invitant à être lus. On va saisir, dans ces recueils,

\subsubsection{Les photographies}

Il y a enfin, les photographies ${ }^{24}$. En Inde, c'est incontournable. Les icônes sont omniprésentes, images, statues, autels, etc. Le pays est recouvert de figurations sacrées. À Pondichéry aussi, dans certains magasins, à côté de Lakshmi (la déesse indienne de la bonne aurovilienne aux "fous de Dieu " autant qu'aux «fous de l'Inde». L'accent est mis sur le symbole.

moins. Eux se reconnaissent à leur attitude : ils marchent souvent pieds nus, les filles portent des jeans, des shorts et t-shirts (avec $45^{\circ}$, les touristes sont en jupes et débardeurs), dans le privé les garçons se mettent en tenue traditionnelle tamoule, torse nu et longhi. Ils conduisent des motos, parfois avec un petit sticker avec le symbole d'Auroville dessus, mais c'est moins répandu. On trouve souvent chez les résidents, sur leur porte ou sur les murs, les symboles protecteurs (et fédérateurs) de Mère et d'Aurobindo.

une parole sacrée, un moment de divination où les fondateurs communiquent par ces objets médiateurs qui les incarnent (c'est leur parole).

$\mathrm{Au}$ cas où les résidents n'ont pas fait l'effort de lire les livres ou les recueils, le News \& Notes (le journal hebdomadaire) débute par un extrait de texte. Il est un rappel aux valeurs, aux aspirations des premiers temps constitutifs de leur unité (identité). fortune), on observe la Mère ou Sri Aurobindo invoqués en tant que protecteurs, mais aussi garants d'une certaine qualité morale du vendeur. Leurs noms sont d'ailleurs associés à une publicité de l'éthique. L'essence distribuée par l'ashram, la nourriture, les encens, les

\footnotetext{
24 Pour compléter nos observations, nous renvoyons le lecteur à la thèse de doctorat de Shanti Pillai de 2005. Bien que certaines informations soient aujourd'hui dépassées, la plupart de ses observations restent pertinentes pour cette thématique. (Pillai, $2005: 314-316)$
} 
produits en général sont dits de meilleure qualité parce que produits « avec conscience ». On observe plus souvent des photographies de Mirra Alfassa que de Sri Aurobindo (même si, à Auroville, ils sont souvent associés) : peut-être parce que c'était surtout elle qui donnait les darshan, ce moment où la divinité regarde les dévots. Certes, Sri Aurobindo donnait lui aussi le darshan avec la Mère avant son décès en 1950. Toutefois selon la tradition hindoue, les déesses sont liées à l'action : ce sont elles qui protègent, purifient25. Aurobindo est l'énergie de l'aspiration. Elle est l'énergie de l'action. À l'image de la déclaration de Mirra Alfassa : « Sans lui, je n'existe pas. Sans moi, il n'est pas manifesté. » (Sri Aurobindo, 1972d : 455-60)

\subsubsection{Les prières et les citations}

Nous avons précédemment évoqué l'utilisation des écrits et des citations des textes des fondateurs comme référents communs et lignes de conduite éthiques de la communauté. Ils sont omniprésents ${ }^{26}$. Ils rappellent à tous le pourquoi d'Auroville. Systématiquement, lors des rencontres, les résidents se posent la question :
"Quelles valeurs veut-on voir à Auroville?». L'idée est d'agir pour " plus grand que soi », que ce soit directement pour leur guru ou pour l'entité de la "ville »(la communauté). Ce leitmotiv commun correspond à une recherche de supériorité morale : «ce n'est pas suffisant, il faut faire mieux. ».

\subsection{Conclusion}

Les éléments prédominants et paradoxaux d'une ritualisation de la dévotion à Auroville ne sont ni respectés ni cautionnés par tous. J'ai vu plus de Ganesh chez les résidents que de photographies de la Mère. Il y a plus de touristes aux bonfires que d'Auroviliens. D'ailleurs, ces derniers se raillent volontiers entre eux sur les questions de l'autorité de la parole de la Mère et leur rapport au sacré27. J'ai rencontré des athées à Auroville comme des personnes attirées par Osho ou Amma, j'ai même discuté avec un croyant aux "reptiliens ». Mais tous se retrouvent dans les valeurs, et ces épandages symboliques ré-enchantent leur monde, leur permettant de vivre un conte au milieu d'une réalité qui n'est pas toujours belle. L'Inde environnante est difficile. Les conditions environnementales sont aussi des rites initiatiques (d'où la durée d'un an demandée aux nouveaux arrivants). Il $y$ a la forêt aussi où il faut se perdre pour trouver les hameaux (les communautés internes à Auroville). Tout cela fait partie des rites de passage à vivre individuellement pour devenir Aurovilien. « Si tu te sens à l'aise : alors Auroville est faite pour toi », m'a-t-on clamé lors de ma première visite. Bien plus que l'argent ou les connaissances, la persévérance, la résistance et l'ardeur sont valorisées.

\footnotetext{
25 Notamment dans le Tamil Nadu lorsqu'on observe les pratiques des cultes des dieux locaux : Aiyanar est toujours présent dans les villages, il est le protecteur majeur, mais c'est Marriamma la déesse qui relève du culte hebdomadaire et qui protège le village des maladies et des catastrophes. Selon moi - mais c'est un point de vue - le « couple » Mère-Aurobindo ressort de cette dialectique ontologique, où Sri Aurobindo œuvre « du dessus » et la Mère est plus proche de la matière, du quotidien..

26 Cf. Pillai, $2005: 147-148$ et 194.

27 Par exemple, une maxime francophone m’a été rapportée par des résidents, lorsque au début de la construction de la ville, les résidents usaient volontiers de « Mère a dit ... » pour tel ou tel sujet, il était d'usage de répondre : « Mère a dit ! Mère a dit : mes carottes oui !» (à entendre : Mère a dit, mes radis : mes carottes !)...
} 
Évidemment, la hiérarchie prédomine comme partout en Inde. Donc, un nouvel arrivant sera toujours en dessous du pionnier dans l'échelle du respect. Mais il sera plus haut que le guest (touriste) voire même que les «périphériens » («Péri Fait Rien » se moquent les francophones), même si cela fait trente ans que ceux-ci viennent en Inde ou qu'ils vivent à Auroville, car ils ne sacrifient pas leur confort personnel et leurs craintes pour travailler à Auroville; ce "sacrifice » et cette dévotion à une entité collective commune sont les plus valorisés.

À Auroville, on observe des mises en scène cultuelles et/ou ritualistes, mais la communauté, par son orientation intra-mondaine, ses croyances flottantes et ses comités rotatifs, empêche l'émergence de figures institutionnelles strictes.

Il n'y a alors ni prêtres ni " grâces d'institution » (Weber, 362), comme le décrit Weber pour évoquer une institutionnalisation du croire. Par ces pratiques flottantes et ces organes de mise à distance, les Auroviliens se préservent du dogmatisme.

Par le réenchantement de leur monde, les résidents agissent comme s'ils se rassuraient psychologiquement de leur bon droit à vivre ici, mais aussi à croire ce qu'ils croient. La foi doit être maintenue par ces petits gestes symboliques, par ces objets et autres rappels ; s'ils ne veulent pas voir s'effondrer dans le doute leurs croyances, entraînés dans la routine du quotidien. Ainsi, il n'y a pas de démarches sectaires ou dogmatiques, mais réellement une association autour de symboles, du vocabulaire et de référents communs. Par ces détours, les Auroviliens, parlant dorénavant le même langage, arrivent à se penser en termes de famille élargie ( On est une grande famille», me dit-on) même s'ils viennent de cultures ou d'univers différents. Par ces techniques, ils se construisent une foi et des valeurs, un univers politico-religieux qui les fait exister dans le monde dans une unité de sens sans pour autant constituer une religion.

En voulant protéger les Auroviliens, qui se trouvaient alors en plein conflit juridique, Stuart Leard avait écarté de son analyse ces observations. Aujourd'hui, il est important de dévoiler ces phénomènes dans une perspective analytique, car elle donne de la matière à repenser les nouvelles pratiques du croire contemporain. En effet, de plus en plus on observe qu'à la suite du rejet du religieux, dans une quête du sens, les individus en appellent à la « spiritualité ». Mais celle-ci ne peut s'exprimer si on ne lui donne pas des éléments sur laquelle s'inscrire : tels les textes, des prières ou des rites. Selon nous, c'est en cela qu'Auroville est exemplaire par sa valorisation de la foi et sa recherche de nouvelles pratiques et de formes de croire. 


\section{Références bibliographiques}

Adilakshmi. La Mère. Schaumburg : Éditions Mother Meera, 1987.

Altglas, Véronique. Le nouvel hindouisme occidental. Paris : CNRS, 2005.

Apostolos-Cappadona, Diane. « Poetry as Yoga: The Spiritual Ascent of Sri Aurobindo », Horizons. 7, 2 (1980) : 265-284.

Aymard, Orianne, Le culte post-mortem des saints dans la tradition hindoue : expérience et institutionnalisation du culte de Ma Anandamayi, 1896-1982. Montréal : Thèse en sciences des religions, UQÀM, Montréal, 2009.

Barlet, C. et Thémanlys. L. La revue cosmique. Paris : Librairie H. Chacornac, 1901-1908.

Barnhart, Robert K. The Barnhart Dictionary of Etymology. New York : H.W. Wilson Co., 1998.

Bhabha, Homi K. Les lieux de la culture. Paris : Payot, 2007.

Bhabha, Homi K., et Jonathan Rutherford. «Le tiers-espace ». Multitudes 26, no 3 (2006) : 95-107. https:// www.cairn.info/revue-multitudes-2006-3-page-95.htm

Beldio, Patrick. Art and Beauty, Opposition and Growth in the Sri Aurobindo Ashram. Thèse en sciences des religions, Catholic University of America, Washington, 2016.

Bhagavad-Gītā. Traduit par Sri Aurobindo, Camille Rao et Jean Herbert. Paris : Librairie d'Amérique et d'Orient Adrien Maisonneuve, 1991.

Boltanski, Luc et Chiapello, Ève. Le Nouvel Esprit du Capitalisme. Paris : Gallimard, 1999.

Bradley, Marc. Le rituel funéraire hindou en contexte diasporique : rite de passage et rite d'ancrage. La communauté tamoule d'origine sri-lankaise de Montréal et Toronto. Thèse en sciences des religions, UQÀM, Montréal, 2015.

Brême David. «L'État Indien et le statut “spirituel” d'Auroville ». Religiologiques 34 (2016) : 295-324. http://www.religiologiques.uqam.ca/no34/34_295-324_Breme.pdf

Brême, David. La figure de la mère, Mirra Alfassa (1878-1973). Une analyse des hybridations culturelles de ses représentations. Thèse en sciences des religions, UQÀM, Montréal, 2017.

Cenkner, William. « Art as Spiritual Discipline in the Lives and Thought of Rabindranath Tagore and Sri Aurobindo Ghose ». Ultimate Reality and Spiritual Discipline, dirigé par James Duerlinger. New York : Paragon House Publishers, 1984, 169-186. 
Champion, Françoise. « Les sociologues de la post-modernité religieuse et la nébuleuse mystiqueésotérique ». Archives de Sciences sociales des Religions 34, n 67/1 (1989) : 155-69. http:// www.jstor.org/stable/41622211.

Champion, Françoise. «La Religion à l'épreuve des Nouveaux Mouvements religieux ». Ethnologie Française 30, n 4 (2000) : 525-33. http://www.jstor.org/stable/40991507.

Champion, Françoise et al. « La nébuleuse mystique-ésotérique ». De l'Émotion en Religion. Paris : Centurion, 1990.

Champion, Françoise et al. Le Sacré Hors Religion. Paris : L'Harmattan, 2007.

David-Néel, Alexandra. Journal de voyage : lettres à son mari. T. 1. Paris : Pocket, 2010.

Denton, Lynn. Female Ascetics in Hinduism. New York University Press, 2004.

Devin, Christine, Retournements. Auroville : Auroville Press Publisher, 2011.

Dumont, Louis. Homo hierarchicus. Paris : Gallimard, 1971.

Eck, Diana. Darśan: Seeing the divine image in India. New York : Columbia University Press, 1998.

Eliade, Mircea. Le sacré et le profane.Paris : Gallimard, 1957.

Fassbender, Franz. Auroville Architecture : Towards New Forms for a New Consciousness. Michigan : Thread Bound, 2014.

Garnoussi, Nadia. De Nouvelles Propositions de Sens Pratiques dans le Domaine de l'Existentiel : Étude sociologique de la "Nébuleuse Psycho-Philo-Spirituelle". Dirigé par J. Baubérot et F. Champion. Paris : Sciences de l'Homme et Société. École pratique des hautes études - EPHE PARIS, 2007.

Geoffrion, Karine. Le rôle du gourou dans un ashram montréalais. Document de travail du GRDU, Udem. 34 (2011): 1-37. http.s://depot.erudit.org/bitstream/003451dd/1/Geoffrion_Document\%20de\%20travail_ GRDU.pdf

Goulet Trishia, Nicole. The lives of Sarada Devi:Gender, renunciation and Hindu politics in colonial India. Thèse en sciences des religions, Université du Manitoba, Winnipeg, 2010.

Heehs, Peter. La Vie de Sri Aurobindo. Paris : Éditions du Rocher, 1989/2003.

Heelas, Paul. The New Age Movement. The Celebration of the Self and the Sacralization of Modernity. Oxford / Cambridge (Massachussets) : Blackwell, 1996.

Hegel, Georg W. F. Leçons sur l'histoire de la philosophie. Paris : Gallimard, 1954.

Higonnet, A. « Femmes et images ». Dans Histoire des femmes en Occident. Vol. 4. Le XIXe siècle, dirigé par Georges Duby et Michelle Perrot. Paris: Plon, 1991, 335-86.

Horassius, Marie. Aire de recherche, ère de la quête du sens. Ethnographie d'une utopie : l'exemple de la communauté internationale d'Auroville. Mémoire de Master en anthropologie, École des hautes études en sciences sociales, Paris, 2012.

Huffer, J. A. Darshan in a Hotel Ballroom : AmritanandamayiMa's (Amma's) Communitees of Devotees in the United States. Thèse en sciences des religions, Chicago University, 2010.

Hulin, M., et C. Maillard. «L'Inde comme lieu des figures de l'Autre ». L'Inde inspiratrice, réception de l'Inde en France et en Allemagne (XIX ${ }^{e}-X X^{e}$ siècles). Strasbourg : Presses Universitaires de Strasbourg, 1996. 
Huta. "My Savitri Work with the Mother”. Invocation. Pondichéry : Sri Aurobindo Ashram, 2001.

Huta. The Story of a Soul. Vol. 2. Part. 2. Pondichéry : Sri Aurobindo Ashram, 2009.

Iyengar, K. R. On the Mother. Pondichéry : Sri Aurobindo International Centre of Education, 1994.

Jouhki, Jukha. Imagining the Other, Orientalism and Occidentalism in Tamil-European Relations in South India. Université de Jyväskylä, 2006. https://jyx.jyu.fi/dspace/handle/123456789/13431

Leard, Stuart. The International Township of Auroville, Tamil Nadu, India : The Routinization of Charisma in a Context of an Inner-Worldly Mystical Orientation. Mémoire de sociologie M.A University of Saskatchewan, Saskatoon, 1993.

Leard, Stuart. Negotiating Modernity: Habermas and the International Township of Auroville. Thèse de sociologie University of Saskatchewan, Saskatoon, 2011.

Macpherson, Crawford B. The Political Theory of Possessive Individualism. NewYork : Oxford University Press, 1962.

Meier, Jane. « BeingAurovilian: Constructions of Self, Spirituality and India in an International Community ». Ethnologielatine. J@rgonia10 (2006): 1-23. https://jyx.jyu.fi/bitstream/handle/ 123456789/20058/Jargonia_10_2006.pdf?sequence=2

La Mère. Entretiens 1956. Pondichéry: Sri Aurobindo Ashram, 1968.

La Mère. Paroles d'autrefois. Pondichéry : Sri Aurobindo Ashram, 1983.

La Mère. La Mère parle d'Auroville. Auroville : Auroville Publications, 1991.

La Mère. Collected Works of The Mother. Vol. 9. Pondichéry: Sri Aurobindo Ashram, 1997.

La Mère. Entretiens 1929-31. Pondichéry: Sri Aurobindo Ashram, 2008.

La Mère. Paroles de la Mère. Vol. 1. Pondichéry : Sri Aurobindo Ashram, 2009a.

La Mère. Pensées et aphorismes. Pondichéry : Sri Aurobindo Ashram, 2009b.

La Mère. Quelques réponses de la Mère. Vol. 2. Pondichéry: Sri Aurobindo Ashram, 2009c.

La Mère. La Charte d'Auroville. (2014). https://www.auroville.org/contents/545.

Meyer, Birgit. "Mediation and the Genesis of Presence:Towards a MaterialApproach to Religion". Conférence du 19 octobre 2012. Université d'Utrecht. www2.hum.uu.nl/onderzoek/lezingenreeks/pdf/ Meyer_Birgit_oratie.pdf.

Minor, Robert N. The Religious the Spiritual and the Secular: Auroville and Secular India. Albany: State University of New York Press, 1999.

Monier-Williams. A Sanskrit-English Dictionary. Delhi : Motilal Banarsidass Publishers, 2005.

Morgan, David. Visual Piety : A History and Theory of Popular Religious Images. Berkeley : University of California Press, 1998.

Morgan, David. The Sacred Gaze : Religious Visual Culture in Theory and Practice. Berkeley : University of California Press, 2005.

Mukherjee, Printhwindra. Les racines intellectuelles du mouvement d'indépendance de l'Inde (1893-1918). Paris : Codex, 2010.

Narad, Interview with Tency. MotherAndSriAurobindo, consulté le 31 décembre 2016. https:// www.youtube.com/watch?v=SLMhaWo2HFw

Pattinson, Stephen. Seeing Things : Deepening Relations with Visual Artifacts. Londres : SCM Press, 2007. 
Pechilis, Karen. The Graceful Guru : Hindu Female Gurus in India and the United States. Oxford : Oxford University Press, 2004.

Piette, Albert. Le Fait religieux : Une théorie de la religion ordinaire. Paris : Economica, 2002.

Pillai, Shanti. Auroville : Philosophy, performance and power in an international utopian community in South India. New York: New York University Press, 2005.

Ray, Paul H. et Sherry, Ruth A. The Cultural Creatives : How 50 Million People Are Changing the World. New York : Harmony Books, 2000.

Ricoeur, Paul. «L'intrigue et le récit historique ». Temps et récit. Tome I. Paris : Le Seuil, 1983.

Ricœur, Paul. Soi-même comme un autre. Paris : Le Seuil, 1990.

Saïd, Edward. L'orientalisme. Paris : Le Seuil, 1978/2005.

Satprem. Carnet d'une apocalypse. Vol. 1. Paris : Institut de Recherche Évolutive, 1973.

Satprem. La Mère : le matérialisme divin. Paris : Robert Laffont, 1976.

Satprem. L’Agenda de Mère. Vol. 1. Paris : Institut de Recherche Évolutive, 1978.

Satprem. L’Agenda de Mère.Vol. 2. Paris : Institut de Recherche Évolutive, 1979a.

Satprem. L’Agenda de Mère. Vol. 3. Paris : Institut de Recherche Évolutive, 1979b.

Satprem. L'Agenda de Mère. Vol.10. Paris : Institut de Recherche Évolutive, 1981a.

Satprem. L'Agenda de Mère. Vol. 11. Paris : Institut de Recherche Évolutive, 1981b.

Satprem. L’Agenda de Mère. Vol.13. Paris : Institut de Recherche Évolutive, 1982.

Satprem. Sri Aurobindo ou l'aventure de la conscience. Paris : Buchet-Chastel, 2003.

Sri Aurobindo. La Synthèse des Yogas. Pondichéry : Sri Aurobindo Ashram, 1914/1970.

Sri Aurobindo. Le Cycle humain, Psychologie du développement social. Pondichéry : Sri Aurobindo Ashram, 1972a.

Sri Aurobindo. L'idéal de l'unité humaine. Paris : Buchet-Chastel, 1972b.

Sri Aurobindo. Letters on Yoga. Vol. 1. Pondichéry: Sri Aurobindo Ashram, 1972c.

Sri Aurobindo. On himself. Vol. 26. Pondichéry : Sri Aurobindo Ashram, 1972d.

Sri Aurobindo. La manifestation du Supramental sur la terre. Pondichéry: Sri Aurobindo Ashram, 1974.

Sri Aurobindo. La pratique du yoga intégral. Paris : Albin Michel, 1976.

Sri Aurobindo. Le Secret du Veda. Pondichéry : Sri Aurobindo Ashram, 1914/1989.

Sri Aurobindo. Les poèmes de Sri Aurobindo. Pondichéry: Sri Aurobindo Ashram, 1981.

Sri Aurobindo. “Letters on Savitri”. Savitri. Pondichéry : Sri Aurobindo Ashram, 1995.

Sri Aurobindo. The Renaissance and Other Essays on Indian Culture. Pondichéry : Sri Aurobindo Ashram, 1997a.

Sri Aurobindo. The Future of Poetry. Pondichéry : Sri Aurobindo Ashram, 1997b.

Sri Aurobindo. Synthesis of Yoga. Pondichéry : Sri Aurobindo Ashram, 1999.

Sri Aurobindo. Savitri (trad. Guy Lafond). Pondichéry: Sri Aurobindo Ashram, 1951/2002. 
Sri Aurobindo. Savitri (trad. Raymond Thépot). Montréal : Christian Feuillette, 1951/2005.

Sri Aurobindo. Letters on Himself and the Ashram. Pondichéry : Sri Aurobindo Ashram, 2011.

Timiri, Himabindu. « Le maternel/spirituel et la question de l'orientalisme dans trois romans québécois contemporains ». Mémoire en études littéraires M.A, UQÀM, Montréal, 2008.

Van Vrekhem, Georges. La Mère, une biographie. Paris : Les Belles Lettres, 2007.

Vishnudevananda, Swami. «Sivananda Yoga Montreal - Lashram Sivananda ». (2017). http:// www.sivananda.org/montreal/ashram-sivananda/

Weber, Jean. Pondichéry et les comptoirs de l'Inde après Dupleix, la démocratie au pays des castes. Paris : Denoël, 1996.

Weber, Max. Économie et société. Paris : Plon, 1971.

Weber, Max. Sociologie de la religion. «1. Les catégories de la sociologie ». Paris : Champs Flammarion, 2013.

Wilfried. The Mother: A short biography. Pondichéry: Sri Aurobindo Society, 1990.

Zeghiche, Sabrina. «Lorsque l'"Occident" se tourne vers l'Inde : analyse de discours de la presse québécoise sur la gestion de la diversité culturelle et religieuse en Inde ». Diversité urbaine. 10, n² (2011) : 7-28. http://id.erudit.org/iderudit/1006423ar 
Ce cahier approche le mouvement spirituel fondé par Sri Aurobindo (1872-1950) et la Mère (1878-1973) en présentant les dynamiques de trois de ses sites : I'Ashram de Sri Aurobindo à Pondichéry, Auroville au Tamil Nadu et le Centre Sri Aurobindo de Montréal. En outre, des chapitres introductifs mettent en contexte le mouvement et résument les biographies de ses fondateurs.

Institut Religioscope

Grand'Places 14 - 1700 Fribourg - Suisse

www.religioscope.org

www.religion.info 